Merci d'utiliser le titre suivant lorsque vous citez ce document :

Lesser, C. (2008-01-07), « Les approaches bilatérales et régionales adoptées pour réduire les obstacles techniques au commerce convergent-elles vers le système commercial multilatéral ? », Éditions OCDE, Paris. http://dx.doi.org/10.1787/242271213605

Les approaches bilatérales et régionales adoptées pour réduire les obstacles techniques au commerce convergent-elles vers le système commercial multilatéral ?

\title{
Caroline Lesser
}

La version originale de ce document a été publiée comme suit :

Lesser, C. (2007-10-18), "Do Bilateral and Regional Approaches for Reducing Technical Barriers to Trade Converge Towards the Multilateral Trading System?", OECD Trade Policy Papers, No. 58, OECD Publishing, Paris. http://dx.doi.org/10.1787/051058723767 
Organisation de Coopération et de Développement Economiques

DIRECTION DES ÉCHANGES ET DE L'AGRICULTURE

Français - Or. Anglais COMITÉ DES ÉCHANGES

Groupe de travail du comité des échanges

LES APPROCHES BILATÉRALES ET RÉGIONALES ADOPTÉES POUR RÉDUIRE LES OBSTACLES TECHNIQUES AU COMMERCE CONVERGENT-ELLES VERS LE SYSTÈME COMMERCIAL MULTILATÉRAL?

Document de travail de l'OCDE sur la politique des échanges $n^{\circ} 58$

Par Caroline Lesser

Tous les documents de travail sur la politique des échanges peuvent être consultés sur le site Internet de l'OCDE à l'adresse suivante : http//www.oecd.org/tad

JT03238442

Ta. 83693

Document complet disponible sur OLIS dans son format d'origine

Complete document available on OLIS in its original format 


\section{EXTRAIT}

Dans le cadre de son travail sur le régionalisme, la Direction des Échanges et de l'Agriculture de l'OCDE, a mené à bien une série d'études comparant les dispositions normatives contenues dans les accords commerciaux régionaux à celles de l'Organisation mondiale du commerce (par exemple dans le domaine des services, de l'investissement et de la concurrence). Le présent document entend compléter les études existantes en examinant les dispositions juridiques relatives aux «obstacles techniques au commerce » (autrement dit, les règlements techniques, les normes et les procédures d'évaluation de la conformité) d'un échantillon d'accords commerciaux bilatéraux et régionaux ainsi que leur degré de similarité et de convergence avec l'Accord de l'OMC sur les obstacles techniques au commerce, et entre eux.

L'étude révèle que la plupart des dispositions relatives aux obstacles techniques au commerce (OTC) qui sont contenues dans les accords bilatéraux et régionaux convergent vers le système commercial multilatéral. Lorsqu'ils sont effectivement mis en œuvre, les accords commerciaux visant une intégration et une coopération réglementaire accrue entre les parties peuvent venir compléter et renforcer l'application de l'Accord de l'OMC sur les obstacles techniques au commerce en donnant une impulsion à l'amélioration des pratiques réglementaires et du dispositif relatif aux OTC des pays membres (par exemple par le biais d'enceintes où peuvent se tenir des consultations ou d'organismes conjoints de normalisation et d'accréditation). Certains éléments viennent toutefois encore s'y opposer. Lorsque des accords commerciaux qui se recoupent promeuvent des critères différents pour l'harmonisation des mesures réglementaires et lorsque les initiatives bilatérales ou régionales sont menées indépendamment des efforts internationaux et détournent l'attention des négociations commerciales multilatérales relatives aux normes, il peut en résulter de nouveaux obstacles tant pour les autorités de tutelle que pour les entreprises. Ces contraintes sont en outre amplifiées dans les pays à faible revenu pâtissant de problèmes liés à l'insuffisance de leurs capacités administratives et techniques. Pour résoudre ces problèmes éventuels, la présente étude formule un certain nombre de recommandations.

Mots clés : obstacles techniques au commerce, OTC, mesures réglementaires, règlements techniques, normes, procédures d'évaluation de la conformité, certification, essais, métrologie, obstacles non tarifaires, Accord de l'OMC sur les obstacles techniques au commerce, équivalence, harmonisation, reconnaissance mutuelle, régionalisme, accords commerciaux régionaux, accords bilatéraux de libre-échange, unions douanières, système commercial multilatéral. 


\section{REMERCIEMENTS}

La présente étude a été rédigée par Caroline Lesser de la Direction des échanges et de l'agriculture de l'OCDE sous la direction de Mme Barbara Fliess et de M. Anthony Kleitz, Chef de division, Libéralisation des échanges et réglementation.

L'auteur souhaite remercier Mme Ana Maria Vallina, responsable du Département du commerce extérieur du ministère chilien de l'Économie, pour sa précieuse contribution à l'étude de cas sur le Chili, M. Sze Gin Low, Directeur général adjoint du département chargé de l'OMC et des négociations commerciales internationales du ministère du Commerce et de l'Industrie de Singapour et ses collègues de SPRING Singapour et de l'Infocomm Development Authority of Singapore pour leurs précieuses observations concernant l'étude de cas sur Singapour et enfin, Mme Zitouni, directrice de service au Département normalisation et de promotion de la qualité du ministère marocain de l'Industrie, du Commerce et de la mise à niveau de l'Économie pour sa contribution à l'étude de cas marocaine. M. Nicolas Sauter a en outre apporté son concours inestimable lors des recherches préliminaires.

L'auteur tient également à remercier MM. Ken Heydon, Visiting Fellow, London School of Economics and Political Science, et David Shortall, consultant indépendant, qui ont relu l'étude et formulé plusieurs suggestions utiles.

Le Groupe de travail du Comité des échanges de l'OCDE a examiné la présente étude et a accepté sa déclassification, sous sa responsabilité, pour permettre une plus large diffusion des observations qui y sont contenues.

Cette étude peut être consultée sur le site Internet de l'OCDE en anglais (et en français) à l'adresse suivante : $\underline{\text { http://www.oecd.org/tad }}$

Droit de reproduction OCDE, 2007

Les demandes d'autorisation aux fins de reproduction ou de traduction de tout ou parti de ce document doivent être adressée à :

Publications de l'OCDE, 2 rue André Pascal, 75775 Paris Cedex 16, France. 


\section{TABLE DES MATIÈRES}

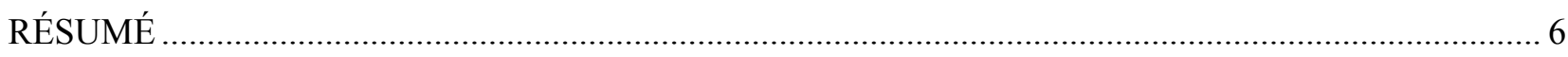

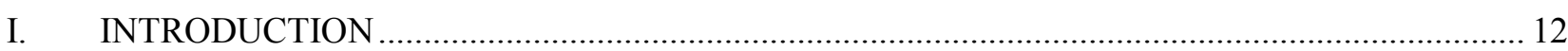

1.1 Contexte

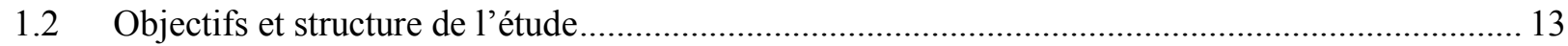

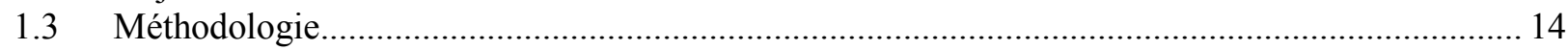

II. APPROCHES POUR RÉDUIRE LES OBSTACLES TECHNIQUES AU COMMERCE .............. 16

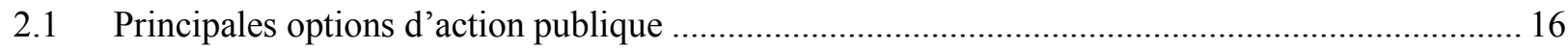

2.2 L'Accord de l'OMC sur les obstacles techniques au commerce .............................................. 18

III. RÈGLES RÉGIONALES EN MATIÈRE D'OTC : CONVERGENCE OU DIVERGENCE PAR RAPPORT AU SYSTÈME COMMERCIAL MULTILATÉRAL ? ................................................... 21

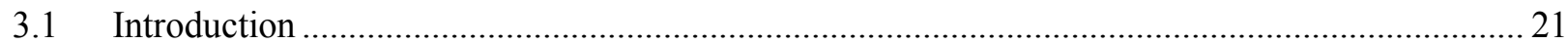

3.2 Vue d'ensemble des dispositions relatives aux OTC d'un échantillon d'accords commerciaux.... 22

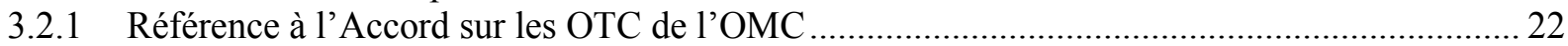

3.2.2 Harmonisation des règlements techniques, normes et procédures d'évaluation de conformité 22

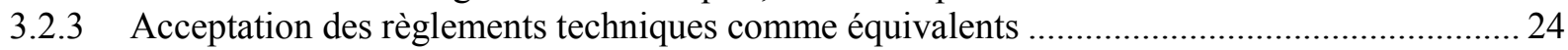

3.2.4 Reconnaissance (mutuelle) des évaluations de la conformité.....................................................24

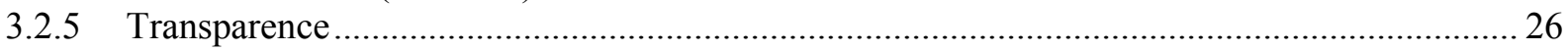

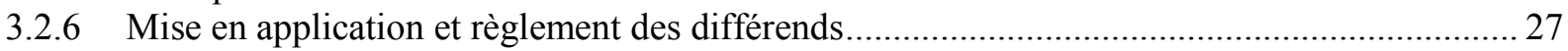

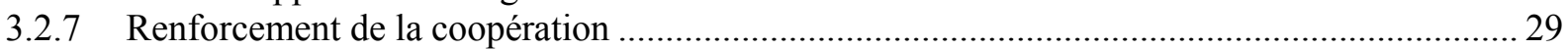

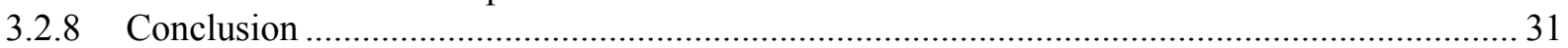

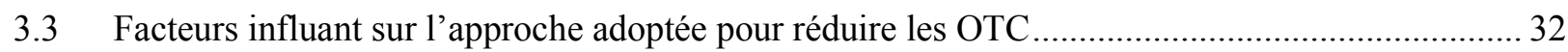

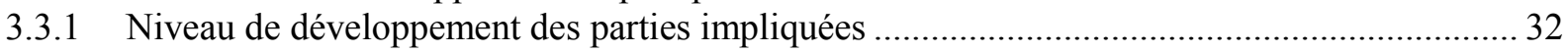

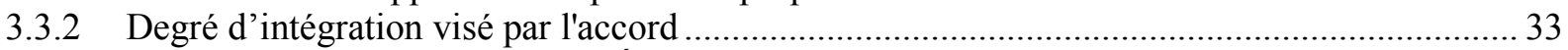

3.3.3 Comparaison de l'approche des États-Unis et de l'Union européenne...................................... 34

3.4 Degré de convergence vers le système commercial multilatéral................................................... 36

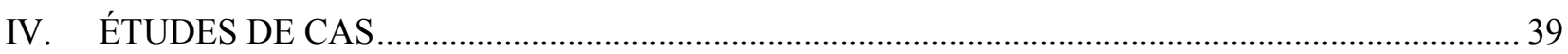

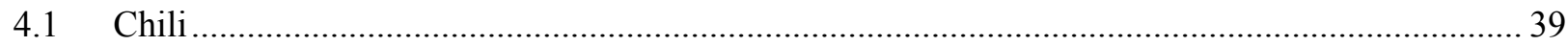

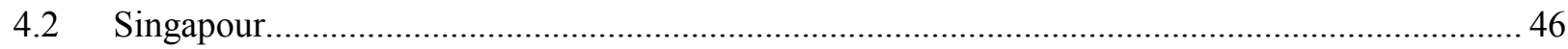

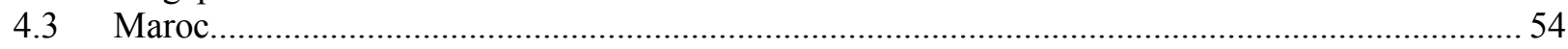

V. CONCLUSIONS PRINCIPALES ET RECOMMANDATIONS …................................................ 60

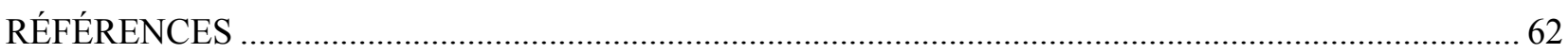

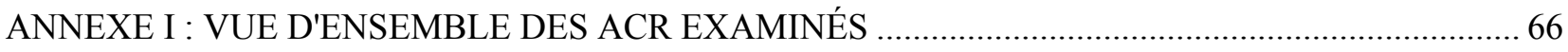

ANNEXE II. INFORMATIONS DE RÉFÉRENCE CONCERNANT LES ÉTUDES DE CAS ................ 72

\section{Graphiques}

Graphique 1. Nombre d'ACR notifiés en vigueur, au mois de mars 2007.............................................. 21

Graphique 2. Approches retenues à l'échelon bilatéral et régional pour traiter la question des OTC ....... 31 


\section{Encadrés}

Encadré 1 Définitions des principaux termes en lien avec les OTC 12

Encadré 2. Principes et dispositions essentiels de l'Accord de l'OMC sur les obstacles techniques au

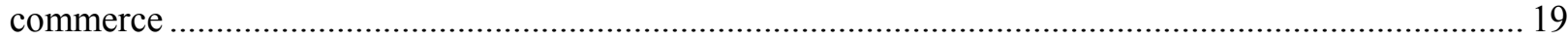

Encadré 3. Types d'accords de reconnaissance mutuelle (ARM) relatifs à l'évaluation de la conformité26

Encadré 4. Comité consultatif sur les normes et la qualité de l'ASEAN (ACCSQ) ................................ 27

Encadré 5. Règlement des différends dans la Communauté andine..................................................... 29

Encadré 6. Assistance en matière d'OTC en vertu de l'Accord de libre-échange de

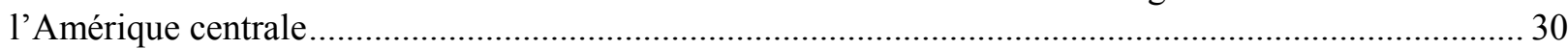

Encadré 7. Politique de normalisation du MERCOSUR …......................................................................... 34

Encadré 8. Approche de l'UE en matière de règlements techniques, normes et procédures d'évaluation

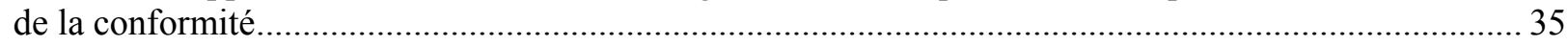

Encadré 9. Comité spécial des règlementations techniques, des normes et des évaluations de la

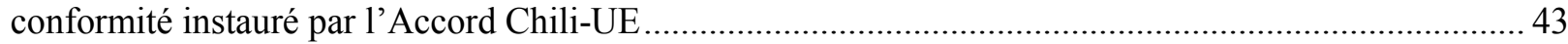

Encadré 10. Responsabilités essentielles du Comité national chilien des obstacles techniques au

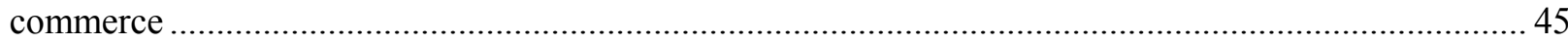

Encadré 11. Approche de 1'APEC pour éliminer les OTC................................................................... 49

Encadré 12. Assistance liée aux OTC aux termes de l'Accord de libre-échange entre les États-Unis

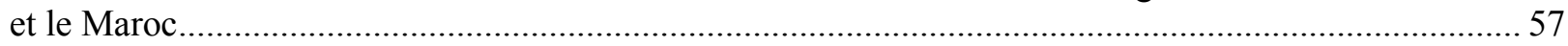

Encadré 13. Réforme du système marocain de normalisation ...........................................................5 


\section{RÉSUMÉ}

1. Si les obstacles tarifaires ont été significativement réduits par les cycles successifs de négociation du GATT et de l'Organisation mondiale du commerce (OMC), les règlements techniques dont le respect est obligatoire, les normes dont le respect est volontaire, et les procédures connexes d'évaluation de la conformité continuent de représenter des obstacles éventuellement importants au commerce (appelés « obstacles techniques au commerce» ou OTC). En tant que mesures réglementaires, ils visent à réaliser des objectifs légitimes d'action publique, par exemple en matière de protection de la santé publique, de la sécurité ou de l'environnement. Cela étant, ces mesures peuvent avoir un caractère discriminatoire vis-àvis des importations, entraîner des restrictions non nécessaires au commerce, causer des dysfonctionnements du marché et entretenir des comportements de recherche de rente. De plus, les différences existant d'un marché à l'autre entre les règlements, les normes et les procédures d'évaluation de la conformité applicables peuvent alourdir les coûts de conformité pour les entreprises présentes sur de nombreux marchés.

2. L'Accord de l'OMC sur les obstacles techniques au commerce («l'Accord sur les OTC de l'OMC ») vise à assurer que ces mesures ne constituent pas des obstacles non nécessaires au commerce, en imposant aux membres de l'OMC des disciplines en matière d'élaboration, d'application, de notification et de révision des règlements techniques, des normes et des procédures d'évaluation de la conformité. Parallèlement, les membres de l'OMC prennent part à un nombre croissant d'unions douanières et d'accords commerciaux bilatéraux, régionaux et plurilatéraux qui contiennent en outre généralement des dispositions relatives aux OTC. En fait, la portée des accords commerciaux conclus au cours de la dernière décennie s'étend bien souvent au-delà des mesures traditionnelles aux frontières qui ont un effet sur les échanges, et visent souvent une intégration économique renforcée entre les parties. Avec la prolifération de ces accords, un même pays devient souvent partie à plusieurs accords de libre-échange. Cela peut entrainer un «enchevêtrement» d'accords qui se recoupent mutuellement, d'où une duplication (ou une contradiction) éventuelle des efforts de libéralisation multilatérale entrepris.

3. La présente étude se penche sur les dispositions relatives aux OTC d'un échantillon d'accords commerciaux bilatéraux, régionaux et plurilatéraux, examine dans quelle mesure ces dispositions vont audelà de l'Accord sur les OTC de l'OMC, et évalue leur convergence ou leur divergence par rapport au système commercial multilatéral. Elle recense en outre les facteurs clés influant sur les différentes approches utilisées dans les accords bilatéraux, régionaux et plurilatéraux pour réduire les OTC et étudie les conséquences de la prolifération des divers engagements pris en matière d'OTC par trois pays se trouvant à différents stades de développement, notamment le Chili, Singapour et le Maroc. Enfin, l'étude propose des recommandations à l'intention des négociateurs de ces accords et, plus généralement, des pouvoirs publics. L'étude se fonde sur une évaluation qualitative de 82 accords commerciaux conclus en Asie, en Amérique du Nord et en Amérique Latine, en Afrique et en Europe, ou entre régions (auxquels participent à la fois des pays développés et des pays en développement). L'échantillon inclut plusieurs accords conclus par les trois pays examinés, ainsi que différents accords ayant été examinés par d'autres organisations (ECDPM, à paraître, Piermartini et Budetta, 2006 et Kotschwar, 2001). L'étude analyse et compare les dispositions OTC, sans quantifier l'effet qu'elles ont sur les flux commerciaux internationaux ou régionaux. 
TAD/TC/WP(2007)12/FINAL

\section{Quelles sont les dispositions «OTC» les plus courantes dans les accords commerciaux bilatéraux, régionaux et plurilatéraux?}

4. $\quad \mathrm{Au}$ nombre des approches de réduction des OTC qui sont le plus souvent encouragées dans les accords examinés figurent la reconnaissance (mutuelle) des résultats des évaluations de la conformité souvent considérée comme moins coûteuse que l'harmonisation des règlements, normes et procédures d'évaluation de la conformité - ainsi que les règles de transparence invitant instamment les membres à se notifier mutuellement les nouveaux règlements techniques et procédures d'évaluation de la conformité en vigueur, ou toute modification des règlements et procédures existants lorsqu'ils ne sont pas conformes aux normes internationales ou sont susceptibles d'avoir un effet sur le commerce. Au nombre des autres approches utilisées, citons notamment l'harmonisation des règlements techniques, des normes et procédures d'évaluation de la conformité par les parties et, dans une moindre mesure, l'acceptation des règlements techniques d'autres parties comme équivalents (lorsque les spécifications techniques qui y sont contenues diffèrent des leurs). Ces différentes approches sont compatibles, de sorte que certains accords comportent parfois des dispositions renvoyant à plusieurs de ces principes. Cela étant, aucun des accords sous revue ne comporte de dispositions prévoyant la reconnaissance mutuelle des règlements techniques et des normes, cette approche n'étant utilisée que dans le marché unique de l'Union européenne.

5. La majorité des accords commerciaux examinés préconise la mise en place d'un comité, organisme ou réseau conjoint qui suivra la mise en œuvre des dispositions sur les OTC, élaborera des propositions en vue de futures améliorations et facilitera les échanges d'information entre les parties. Un organisme de ce type peut avoir un caractère sectoriel (comme dans le cas de l'accord commercial conclu entre le Chili et le Canada) ou être remplacé par la désignation de «coordinateurs » ou de "points de contact » dans chaque pays partie à l'accord (comme le prévoient certains accords de libre-échange conclus par les États-Unis). De la même façon, la majorité des accords commerciaux bilatéraux, régionaux et plurilatéraux examinés contiennent des dispositions régissant le règlement des différends liés aux OTC, qui invitent pour la plupart les parties à se concerter en cas de désaccord. Un nombre plus limité d'accords (comme l'Association latino-américaine d'intégration ou ALADI) envisage aussi le recours à des sousgroupes techniques régionaux et à des groupes de travail ad hoc qui peuvent formuler des recommandations non contraignantes. Enfin, près de $40 \%$ de tous les accords commerciaux examinés contiennent des dispositions relatives à l'assistance technique, en vertu desquelles les parties les plus développées s'engagent à soutenir les parties à plus faible revenu en dispensant à leurs agents publics et aux négociants des formations sur les OTC, en les aidant à renforcer leurs institutions pour améliorer la transparence et à consolider leur infrastructure physique d'essais et de certification des produits d'une part et d'accréditation des organismes d'évaluation de la conformité d'autre part (comme l'aide apportée par l'UE au Chili par exemple).

6. Il est malheureusement difficile d'évaluer les progrès d'ensemble accomplis dans la réduction effective des OTC au niveau bilatéral ou régional, en raison du petit nombre de rapports de mise en œuvre et de documents disponibles sur le sujet, notamment en ce qui concerne les accords bilatéraux de libreéchange. Si la plupart des accords bilatéraux ou régionaux prévoit la mise en place de comités conjoints chargés de suivre la mise en œuvre des dispositions OTC, peu nombreux sont ceux qui mettent à disposition du grand public des rapports d'étape relatifs à la mise en application (à l'exception des groupements régionaux et des unions douanières). La documentation disponible donne toutefois à penser que les efforts de libéralisation des OTC entrepris, notamment en matière d'harmonisation des règlements et des normes et de reconnaissance mutuelle des procédures d'évaluation de la conformité sont davantage susceptibles d'être effectivement mis en æeuvre dans les pays relativement développés (à revenu élevé ou moyen supérieur), car les pays pauvres ne sont souvent pas dotés des institutions nationales qui sont nécessaires pour faire appliquer ces dispositions ou pâtissent d'une absence de volonté politique à haut niveau pour renforcer leurs institutions. 


\section{Est-ce que ces dispositions vont au-delà de l'Accord sur les OTC de l'OMC ?}

7. L'analyse montre que si, dans l'ensemble, la majorité des accords examinés ne comporte pas de dispositions plus strictes que l'Accord sur les OTC de l'OMC, certains accords commerciaux comportent des caractéristiques qui vont au delà des dispositions de l'OMC. Ces dispositions prévoient principalement l'acceptation des règlements techniques comme équivalents et la reconnaissance (mutuelle) des procédures et organismes d'évaluation de la conformité, et imposent aux signataires d'expliquer aux autres parties, quand elles en font la demande, les raisons d'une non-équivalence ou non-reconnaissance. En outre, un petit nombre de parties (surtout des pays développés, comme les pays de l'UE et les pays les plus développés de l'ASEAN et de l'APEC - tel Singapour) a conclu, parallèlement aux accords bilatéraux, régionaux et plurilatéraux, des accords de reconnaissance mutuelle des résultats des évaluations de la conformité pour certains secteurs donnés (équipements de télécommunications, électriques, électroniques et médicaux notamment).

8. En réalité, il semble que trois facteurs essentiels aient une incidence sur l'approche utilisée pour réduire les OTC dans les accords commerciaux bilatéraux, régionaux et plurilatéraux :

- Le niveau de développement des parties : Les dispositions sur l'harmonisation des règlements techniques et des normes et, surtout, de reconnaissance des résultats des évaluations de la conformité seront plus probablement conclues entre pays similaires, ayant un niveau de développement comparable. La reconnaissance mutuelle des évaluations de la conformité, notamment, est plus probable entre pays dotés de capacités techniques similaires, mutuellement confiants dans leurs procédures réglementaires, leurs institutions et leur infrastructure respectives.

- Le degré d'intégration visé par l'accord: Les accords visant une intégration économique (et politique) renforcée -- comme les unions douanières, les accords destinés à instaurer un marché unique et les accords d'association économique-- poursuivent souvent des objectifs allant au-delà de l'Accord sur les OTC de l'OMC et cherchent à harmoniser (graduellement) les règlements techniques, les normes et les procédures d'évaluation de la conformité et, jusqu'à un certain point, les mesures métrologiques. Nombre d'accords commerciaux prévoient notamment la mise en place de mécanismes de suivi et de coopération conjoints, tels des organismes de normalisation et d'accréditation régionaux (comme par exemple le comité consultatif de l'Association des Nations de l'Asie du Sud-Est ou ASEAN sur les normes et la qualité), que n'impose pas l'Accord sur les OTC de l'OMC.

- L'implication de l'UE ou des États-Unis : Dans les accords commerciaux conclus par les ÉtatsUnis, la libéralisation en matière d'OTC peut prendre plusieurs formes, notamment l'acceptation comme équivalents des règlements techniques de l'autre partie, l'alignement sur les normes internationales et la reconnaissance des résultats des évaluations de la conformité par l'intermédiaire de mécanismes très variés. (Leurs dispositions vont souvent au-delà des règles de l'OMC, en imposant aux parties de fournir une explication en cas de non-reconnaissance des règlements et des résultats des évaluations de la conformité ou des organismes qui s'en chargent). En revanche, la plupart des accords commerciaux conclus par l'UE privilégient l'harmonisation. L'harmonisation avec les règlements, normes et procédures d'évaluation de la conformité de l'Union est l'approche qui est favorisée dans les accords conclus avec les pays candidats à l'adhésion ou les pays «voisins» (comme les pays du Partenariat euro-méditerranéen, EUROMED, par exemple), tandis que les accords commerciaux conclus avec des pays plus lointains comme le Chili privilégient la convergence avec les normes internationales. 


\section{Y-a-t-il une convergence ou une divergence par rapport au système commercial multilatéral ?}

9. On peut dire que la majorité des dispositions relatives aux OTC contenues dans les accords commerciaux bilatéraux, régionaux et plurilatéraux convergent vers le système commercial multilatéral et le renforce. Premièrement, la plupart des accords conclus après 1995 réaffirme les droits et les obligations des parties aux termes de l'Accord sur les OTC de l'OMC et fait référence aux objectifs de cet Accord. Deuxièmement, les règles d'harmonisation encouragent, dans leur grande majorité, la convergence vers les normes et guides internationaux. Troisièmement, la plupart des engagements en matière de transparence figurant dans les accords commerciaux sont, en substance, similaires à ceux de l'Accord sur les OTC de l'OMC. Les dispositions imposant aux parties de fournir une explication en cas de non-reconnaissance de mesures à caractère normatif visent à supprimer toute discrimination et à faciliter encore plus les échanges. On peut donc considérer qu'elles convergent et renforcent l'Accord de l'OMC. Il en va de même pour les mécanismes et dispositifs favorisant une plus grande coopération entre les parties (par exemple par le biais d'une normalisation et d'une accréditation conjointe ou d'une coopération dans le domaine de la métrologie et de l'assistance technique). Ces initiatives peuvent en outre conforter et renforcer la mise en œuvre de l'Accord sur les OTC de l'OMC et donner une impulsion à l'amélioration des pratiques réglementaires dans les pays parties aux accords commerciaux bilatéraux, régionaux et plurilatéraux, contribuant ainsi au renforcement du système commercial multilatéral.

10. Il peut y avoir des risques de divergence lorsque les parties sont invitées à élaborer des règlements, normes et procédures d'évaluation de la conformité régionaux. Si de telles mesures reposent souvent sur des normes internationales ou visent à combler les lacunes existantes lorsqu'il n'existe pas de normes internationales (ou lorsque celles-ci sont considérées comme inappropriées), la coexistence de différents critères d'harmonisation et la multiplicité des règlements techniques, normes et procédures peuvent parfois compliquer les relations commerciales internationales. La littérature existante souligne aussi que si les accords de reconnaissance mutuelle sont conformes aux principes de l'OMC (encourageant la conclusion de tels accords), ils peuvent, dans un petit nombre de cas, avoir un impact défavorable sur les exportations de pays tiers et être de ce fait contraire à l'esprit de non-discrimination. Ces effets peuvent néanmoins être minimisés par une plus grande transparence et une simplification des modalités d'adhésion des pays tiers.

11. En conclusion, le risque le plus important pour le système multilatéral peut découler du fait que les capacités humaines et institutionnelles sont détournées des efforts de libéralisation multilatéraux au profit d'initiatives régionales. Le manque de moyens humains et financiers et les capacités insuffisantes qui en résultent pour pouvoir prendre activement part aux négociations de l'OMC et aux activités de normalisation internationale d'une part, et aux négociations, activités de libéralisation et institutions régionales d'autre part, constituent une préoccupation importante, surtout pour les pays en développement. La présente étude souligne que les pays en développement (à faible revenu) ont souvent du mal à se conformer aux dispositions bilatérales ou régionales relatives aux OTC vis-à-vis desquelles ils ont pris des engagements et qu'ils ne parviennent pas toujours à mener à bien, comme ils l'avaient prévu au départ, une coopération en matière de réglementation.

\section{Principales recommandations}

12. Pour atténuer ces problèmes de capacités, assurer que les accords bilatéraux, régionaux et plurilatéraux renforcent le système commercial multilatéral et favoriser une plus grande cohérence des chapitres relatifs aux OTC figurant dans les différents accords, les parties pourraient :

- Apporter une assistance effective aux pays à faible revenu : outre le fait d'inciter les parties à participer aux négociations commerciales et aux processus de normalisation régionaux et internationaux, les accords commerciaux bilatéraux, régionaux et plurilatéraux devraient aussi les encourager, si on leur en fait la demande, à fournir des formations aux représentants des pouvoirs 
publics et associations professionnelles des parties moins développées afin qu'ils soient mieux informés, aient une meilleure connaissance des questions relatives aux OTC et soient en mesure de mieux évaluer les coûts et avantages des différentes possibilités d'action publique destinées à réduire les OTC. De plus, les mécanismes régionaux de consultation et de suivi devraient être renforcés, afin que les engagements pris à l'échelon régional en matière d'OTC puissent être mieux respectés. Enfin, les principales infrastructures et institutions relatives aux OTC devraient être consolidées pour renforcer la confiance dans la fiabilité des procédures et dispositifs des pays en développement et favoriser la réduction des OTC à l'échelon régional et multilatéral.

- Renforcer la transparence et la disponibilité des informations relatives aux efforts accomplis à l'échelon bilatéral et régional en matière d'OTC et des mesures à caractère normatif (obligatoires ou facultatives) en vigueur dans les pays parties aux accords bilatéraux, régionaux et plurilatéraux, pour éviter une redondance des efforts menés et informer les exportateurs dans les parties et les pays tiers. Les parties aux accords devraient s'attacher à diffuser davantage d'informations relatives au respect des obligations qui y sont contenues. De plus, les points d'information devraient être incités à rassembler et à diffuser (à titre gracieux) toutes les informations utiles concernant les OTC à l'échelon régional ou bilatéral, par l'intermédiaire d'un site Internet par exemple. Parallèlement, il convient de poursuivre les efforts menés à l'échelle internationale pour améliorer la collecte des données et la transparence des mesures relatives aux OTC en cours de négociation ou en vigueur dans différents pays/différentes régions (par l'entremise du Comité de l'OMC sur les OTC et du Comité de l'OMC sur les accords commerciaux régionaux, par exemple).

- Encourager l'adoption de "dispositions types" dans les chapitres relatifs aux OTC des accords commerciaux bilatéraux, régionaux et plurilatéraux afin d'éviter toute contradiction entre ces accords et d'assurer leur cohésion avec le système commercial multilatéral. Les récents efforts accomplis par la Coopération économique Asie-Pacifique (APEC) pour rationaliser et simplifier les dispositions relatives aux OTC des différents accords bilatéraux et régionaux constituent un bon exemple à cet égard. Idéalement, l'élaboration des chapitres sur les OTC contenus dans les accords commerciaux devrait être le fruit d'une étroite coordination à l'échelon national et d'un dialogue entre les négociateurs de ces accords, les experts de l'OMC, les autorités de tutelle, les associations professionnelles œuvrant à l'élaboration, à l'application et à la mise en œuvre des règlements techniques, des évaluations de la conformité et des normes volontaires à l'échelon national, régional et international. Pour assurer cette convergence, les accords commerciaux bilatéraux, régionaux et plurilatéraux devraient systématiquement :

1. réaffirmer les droits et obligations des parties aux termes de l'Accord sur les OTC de l'OMC, ainsi que les objectifs visés par cet accord,

2. imposer que les normes et guides internationaux (lorsqu'ils existent) servent de base pour élaborer des règlements et procédures d'évaluation de la conformité nationaux et régionaux. Les accords pourraient indiquer aux parties comment appliquer au mieux ces normes internationales à l'échelon régional,

3. encourager une convergence de vue entre les parties aux accords commerciaux bilatéraux, régionaux et plurilatéraux sur les questions internationales en matière d'OTC afin de leur donner davantage de «voix» et renforcer leur pouvoir de négociation dans les enceintes internationales,

4. promouvoir effectivement une coordination et une coopération plus intenses en matière de réglementation entre les parties aux accords en vue de favoriser la réduction des OTC à l'échelon régional et multilatéral et d'encourager l'adoption de «bonnes pratiques» réglementaires par celles-ci. 
- Chercher à «internationaliser » les initiatives régionales mises en aeuvre avec succès dans certains secteurs prioritaires. Dans les secteurs mondialement intégrés, caractérisés par une part élevée d'échanges intra-sectoriels, comme les équipements de télécommunications et d'informatique, il faudrait, idéalement et à chaque fois que cela est possible, que les accords de reconnaissance mutuelle aient une portée non seulement régionale mais aussi internationale. De plus, il conviendrait d'encourager les accords multilatéraux entre les organismes d'évaluation de la conformité du secteur privé. 


\section{INTRODUCTION}

\subsection{Contexte}

13. Si les obstacles tarifaires tous pays tiers (NPF) ont été substantiellement réduits durant les cycles de négociation successifs du GATT et de l'Organisation mondiale du commerce (OMC), diverses mesures, comme les règlements techniques, les normes et les procédures connexes d'évaluation de la conformité continuent de représenter d'importants obstacles éventuels au commerce. Ces mesures sont conjointement appelées «obstacles techniques au commerce» (OTC, voir Encadré 1). En tant que mesures réglementaires, elles visent à réaliser des objectifs légitimes d'action publique, notamment en matière de protection de la sécurité du pays, de la santé publique et de la sécurité des personnes et de l'environnement. En outre, elles assurent l'interopérabilité des composantes des produits qui sont vitales au fonctionnement de la chaîne de production mondiale et donnent aux consommateurs des informations sur les produits. La Banque mondiale (2004), souligne ainsi que les futurs règlements et normes publics et privés peuvent donner une impulsion et une orientation aux investissements réalisés dans les entreprises ainsi qu'à une modernisation de la chaîne d'approvisionnement et inciter encore plus les intéressés à adopter de meilleures pratiques dans le domaine de la production agricole et industrielle.

\section{Encadré 1. Définitions des principaux termes en lien avec les OTC}

Les règlements techniques et les normes définissent certaines caractéristiques spécifiques des produits, comme leur taille, leur forme, leurs fonctions, leurs propriétés d'emploi, leur étiquetage ou leur emballage, ainsi que les normes connexes en matière de procédés et de production. Les règlements techniques sont élaborés par les pouvoirs publics, leur application est obligatoire et des organismes publics sont chargés de les faire respecter. En revanche, les normes sont souvent définies par des organismes et associations non gouvernementaux (comme les organismes de normalisation et les associations professionnelles par exemple), leur application est volontaire et c'est le marché qui les fait respecter.

Les procédures d'évaluation de la conformité confirment qu'un produit ou procédé de production est conforme aux obligations techniques ou aux normes du pays destinataire. Elles comprennent, entre autres, les procédures d'échantillonnage, d'essai et d'inspection ; les procédures d'évaluation, de vérification et d'assurance de la conformité des produits et les procédures d'enregistrement, d'accréditation et d'homologation des organismes d'évaluation de la conformité. Par accréditation d'une institution d'évaluation de la conformité, on entend l'attestation de la compétence de cette institution pour mener à bien des missions spécifiques d'évaluation de la conformité.

La métrologie légale concerne les exigences légales en matière de mesures et instruments de mesure. Elle vise à garantir la qualité et la crédibilité nécessaire des mesures dans le cadre des contrôles réalisés par ou sous la responsabilité de l'État dans les domaines de la santé, de la sécurité et de l'environnement.

Les mesures à caractère normatif renvoient à tous les termes susmentionnés.

Source : OMC - Accords commerciaux régionaux - Portail ; http://www.oiml.org

14. Toutefois, ces mesures peuvent avoir explicitement ou implicitement des effets discriminatoires sur les importations, induire des restrictions non nécessaires au commerce ou occasionner des dysfonctionnements du marché et entretenir des comportements de recherche de rente. Tel peut être le cas lorsque les règlements techniques et les normes d'un pays importateur ne sont pas bien calibrés par rapport à l'objectif spécifique qu'ils visent à atteindre, sont appliqués de manière arbitraire ou mis en œuvre par le biais de procédures d'essais ou de certification obligatoires insuffisamment claires, qui n'ont pas été suffisamment diffusées ou qui sont difficiles d'accès ou onéreuses pour les constructeurs ou producteurs étrangers (Kotschwar, 2001). Plus généralement, les différences d'un pays à l'autre, entre les règlements techniques, normes et procédures d'évaluation connexes d'évaluation de la conformité peuvent augmenter les coûts de mise en conformité pour les entreprises implantées sur de nombreux marchés. Ces coûts sont particulièrement décourageants pour les petites et moyennes entreprises (PME). Même s'il est difficile de 
les estimer avec précision, la nécessité de se conformer à des règlements techniques, normes et procédures d'évaluation de la conformité dissemblables d'un pays à l'autre induit pour les producteurs et les exportateurs des coûts directs et indirects ${ }^{1}$. Dans les pays en développement, en particulier, les PME sont confrontées à des problèmes considérables pour surmonter les OTC, faute d'infrastructure institutionnelle et technique et de capacités suffisantes leur permettant de se conformer aux règlements techniques et aux normes sur le point d'être finalisés et pour apporter la preuve de leur conformité. De plus, de nombreux exportateurs des pays en développement n'ont souvent pas connaissance des mesures à caractère normatif qu'ils doivent respecter pour prendre pied sur des marchés étrangers (OCDE, 2005a, Wilson 2004 et ECDPM, à paraître).

15. L'Accord de l'OMC sur les obstacles techniques au commerce («l'Accord sur les OTC de l'OMC ») vise à assurer que les règlements techniques, normes et procédures d'évaluation de la conformité ne constituent pas des obstacles non nécessaires au commerce, en indiquant les disciplines à suivre en matière d'élaboration, d'application, de notification et de suivi de ces mesures dans les pays membres de l'OMC. Parallèlement, les membres de l'OMC prennent de plus en plus part à des unions douanières et des accords de libre-échange bilatéraux, régionaux et plurilatéraux (appelés ici accords commerciaux régionaux ou «ACR ») qui comportent aussi souvent des dispositions relatives aux OTC. Dans la mesure où ils sont conclus entre des pays similaires, ces ACR pourraient en principe comporter des dispositions relatives aux OTC allant au-delà des disciplines de 1'OMC.

\section{$1.2 \quad$ Objectifs et structure de l'étude}

16. S'il existe une littérature considérable sur les avantages et les inconvénients des ACR du point de vue de leur impact sur la prospérité économique et de leur effet sur la création et le détournement des échanges, il n'existe guère d'analyse sur la nature de ces accords et leur convergence ou divergence par rapport aux dispositions normatives à l'échelon multilatéral. Les travaux menés à ce jour par l'OCDE comparent les dispositions normatives des ACR à celles de l'OMC dans un certain nombre de domaines. ${ }^{2}$ La présente étude vise à compléter ces travaux en examinant les dispositions juridiques relatives aux OTC d'un échantillon d'ACR ainsi que leur similarité avec et leur convergence vers l'Accord sur les OTC de l'OMC et entre eux. Elle examine et compare leurs dispositions normatives sans en quantifier l'effet sur les flux commerciaux internationaux et régionaux ${ }^{3}$.

17. L'étude s'articule comme suit :

1. Selon les conclusions de l'étude OCDE (1996), les différents règlements techniques et normes applicables, conjugués au coût des procédures d'essai et de certification de la conformité, pourraient représenter de $2 \%$ à $10 \%$ du coût global de production. Les coûts directs comprennent le recrutement de conseillers techniques pour interpréter les règlements étrangers, des investissements plus élevés dans les unités de production et la réalisation de procédures de certification supplémentaires. Les coûts indirects comprennent des frais de stocks et d'approvisionnement plus élevés. En outre, l'existence de normes et règlements différents réduit l'aptitude des entreprises à augmenter leur productivité en réalisant des économies d'échelle.

2. L'étude OCDE (2003) compare les dispositions normatives des accords commerciaux régionaux à celles de l'OMC pour dix domaines particuliers : les services, la mobilité de la main-d'œuvre, l'investissement, la politique de la concurrence, la facilitation des échanges, les marchés publics, les droits de propriété intellectuelle, la défense commerciale, l'environnement et les règles d'origine.

3. Ceci s'explique par le fait que de nombreux accords examinés, notamment ceux qui se caractérisent par des dispositions relatives aux OTC allant au-delà des règles de l'OMC, n'ont été mis en œuvre que récemment. De plus, on évaluera mieux l'impact sur les flux commerciaux en tenant compte de l'ensemble des dispositions contenues dans les ACR, et non pas seulement de celles qui ont trait OTC. 
- Le chapitre II expose succinctement les principaux principes et approches adoptés pour réduire les OTC et met en relief les grandes disciplines énoncées dans l'Accord sur les OTC de l'OMC.

- Le chapitre III examine les dispositions relatives aux OTC contenues dans un échantillon d'ACR et souligne les cas où ces dispositions vont au-delà des disciplines sur les OTC de l'OMC. Il établit ensuite une distinction entre différents facteurs ayant une incidence sur l'approche utilisée pour réduire les OTC et examine si ces dispositions viennent généralement compléter le système commercial multilatéral ou lui font concurrence.

- Le chapitre IV présente trois études de cas consacrées à des pays se trouvant à différents stades de développement, ayant conclu, avec de grands partenaires commerciaux et avec des pays voisins, des ACR comportant des dispositions relatives aux OTC. Il s'agit du Chili, pays à revenu moyen supérieur, de Singapour, pays à revenu élevé et du Maroc, pays à revenu moyeninférieur. ${ }^{4} \mathrm{Y}$ sont examinés un certain nombre de dispositions relatives aux OTC vis-à-vis desquelles le pays a pris des engagements ainsi que l'infrastructure institutionnelle mise en place pour traiter les questions se rapportant aux OTC.

- Le chapitre $\mathrm{V}$ formule des conclusions et recommandations clés.

- L'annexe I contient des informations relatives à l'échantillon d'ACR sous revue.

- L'annexe II comporte des informations de référence sur les études de cas en question.

\subsection{Méthodologie}

18. L'étude propose une évaluation qualitative de la couverture des OTC dans les ACR ayant un effet sur le commerce de marchandises, en s'appuyant sur un échantillon de 82 accords conclus en Asie, en Amérique du Nord, en Amérique latine, en Afrique et en Europe, ainsi qu'entre les différentes régions. Les ACR examinés concernent à la fois des pays développés et en développement et recouvrent différents types d'accords (accords de libre-échange, unions douanières, accords préférentiels). Les ACR sont évalués par rapport à l'Accord sur les OTC de l'OMC et comparés entre eux, en se fondant sur 7 critères (cf. Cadre analytique présenté au Tableau 1). L'analyse s'appuie sur le texte juridique d'un échantillon de 24 ACR (examinés dans le cadre des études de cas par pays) et sur les examens récents de différents ACR effectués par d'autres organisations. ${ }^{5}$ Les études de cas par pays analysent et comparent les dispositions relatives aux OTC contenues dans différents ACR et s'appuient sur une analyse de la littérature disponible et sur les informations fournies par les responsables et les experts des pays examinés.

4. La classification des pays se fonde sur les données de la Banque mondiale. Au sein de l'OMC, ces pays sont des pays en développement.

5. Piermartini et Budetta (2006) examinent 58 ACR contenant des dispositions sur les OTC, couvrant toutes les zones géographiques et catégories de pays; Kotschwar (2001) examine 15 ACR en Amérique du Nord et en Amérique du Sud et ECDPM (à paraître) 4 ACR de l'UE. La présente étude ne s'attarde pas sur l'ALENA et le marché unique de l'Union européenne, car ces accords sont déjà analysés très détail dans la documentation existante. 
TAD/TC/WP(2007)12/FINAL

Tableau 1. Cadre analytique utilisé pour examiner les dispositions relatives aux OTC des ACR

\begin{tabular}{|c|c|}
\hline Catégorie & Questions \\
\hline $\begin{array}{l}\text { 1. Référence à } \\
\text { l'Accord sur les OTC } \\
\text { de l'OMC }\end{array}$ & $\begin{array}{l}\text { - } \quad \text { L'accord fait-il référence à l'Accord de l'OMC (ses objectifs, règles et/ou dispositions) ? } \\
\text { - } \quad \text { Le chapitre sur les OTC de l'accord vise-t-il des objectifs d'une plus grande portée ? }\end{array}$ \\
\hline 2. Harmonisation & $\begin{array}{l}\text { - L'accord impose-t-il aux parties ou les encourage-t-il à harmoniser leurs règlements techniques, } \\
\text { normes et procédures d'évaluation de la conformité ? } \\
\text { - Encourage-t-il l'utilisation des normes et guides internationaux et/ou régionaux comme base à } \\
\text { l'élaboration de règlements, normes et procédures d'évaluation de la conformité nationaux? }\end{array}$ \\
\hline $\begin{array}{l}\text { 3. Acceptation des } \\
\text { règlements } \\
\text { techniques comme } \\
\text { équivalents }\end{array}$ & $\begin{array}{l}\text { - L'accord impose-t-il aux parties ou les encourage-t-il à accepter comme équivalents les règlements } \\
\text { techniques et les normes des autres parties? } \\
\text { - Les invite-t-il à expliquer les raisons pour lesquelles elles n'acceptent pas comme équivalents les } \\
\text { règlements techniques des autres parties? }\end{array}$ \\
\hline $\begin{array}{l}\text { 4. Reconnaissance } \\
\text { (mutuelle) des } \\
\text { évaluations de la } \\
\text { conformité }\end{array}$ & $\begin{array}{l}\text { - L'accord préconise-t-il une reconnaissance (mutuelle) des résultats des évaluations de la conformité ? } \\
\text { - } \quad \text { Impose-t-il aux parties d'expliquer leurs raisons en cas de non-reconnaissance ? } \\
\text { - S'accompagne-t-il d'un accord (distinct) de reconnaissance mutuelle ou encourage-t-il la conclusion } \\
\text { de tels accords? }\end{array}$ \\
\hline 5. Transparence & $\begin{array}{l}\text { - } \quad \text { 'accord contient-il des dispositions sur la transparence ? } \\
\text { - } \quad \text { Impose-t-il aux membres de procéder à des consultations et de notifier aux autres parties les } \\
\text { règlements et procédures à l'échelon bilatéral ou régional avant leur adoption? } \\
\text { - } \quad \text { Fixe-t-il un délai de réception des observations des autres parties ? Ce délai est-il supérieur à } \\
60 \text { jours? } \\
\text { - Impose-t-il la mise en place d'un point d'information (distinct) ? }\end{array}$ \\
\hline $\begin{array}{l}\text { 6. Mise en application } \\
\text { et règlement des } \\
\text { différends }\end{array}$ & $\begin{array}{l}\text { - L'accord préconise-t-il la mise en place d'un organisme régional (par exemple un comité) sur les OTC, } \\
\text { et invite-t-il les parties à y participer pour assurer le suivi des engagements pris en matière d'OTC ? } \\
\text { - Contient-il des dispositions spécifiques de règlement des différends en lien avec les OTC à l'échelon } \\
\text { régional? } \\
\text { - Prévoit-il la tenue de consultations et la formulation de recommandations, ou un mécanisme plus } \\
\text { formel de règlement des différends à l'échelon régional ? }\end{array}$ \\
\hline $\begin{array}{l}\text { 7. Renforcement de } \\
\text { la coopération }\end{array}$ & $\begin{array}{l}\text { - } \quad \text { Contient-il des dispositions spécifiques d'assistance technique ? } \\
\text { - } \quad \text { Prévoit-il une coopération en matière de métrologie? }\end{array}$ \\
\hline
\end{tabular}

Source : Ce cadre analytique s'inspire de Sampson et Woolcock, 2003 et de Piermartini et Budetta, 2006. 


\section{APPROCHES POUR RÉDUIRE LES OBSTACLES TECHNIQUES AU COMMERCE}

19. En vue de traiter la question des OTC, les pays ont inclus, dans les accords commerciaux internationaux, des dispositions s'efforçant de trouver un équilibre entre les objectifs publics légitimes et l'accroissement des flux commerciaux internationaux. Ces accords ou dispositions se rapportant aux OTC reposent sur un certain nombre de principes ou d'options essentiels (compatibles) visant à réduire les OTC, bien que la portée de chacun d'entre eux puisse varier. Ce chapitre expose succinctement les approches retenues par les pouvoirs publics et les autorités de tutelle en vue de réduire les obstacles techniques au commerce et met en relief les obligations et recommandations contenues dans l'Accord sur les OTC de l'OMC. La présente partie n'entend pas analyser en détail les avantages et difficultés de chaque approche, mais plutôt fournir des informations de référence en vue d'évaluer les approches et les différentes options d'action publique utilisées dans les accords commerciaux bilatéraux, régionaux et plurilatéraux et les unions douanières. ${ }^{6}$

\subsection{Principales options d'action publique}

Tableau 2. Principales options d'action publique vis-à-vis des OTC ${ }^{7}$

\begin{tabular}{|c|c|c|}
\hline Principe essentiel & Portée & $\begin{array}{c}\text { Principaux avantages pour les } \\
\text { négociants des pays parties à } \\
\text { l'accord }\end{array}$ \\
\hline $\begin{array}{l}\text { Harmonisation des } \\
\text { règlements techniques, } \\
\text { normes et procédures } \\
\text { d'évaluation de la } \\
\text { conformité }\end{array}$ & $\begin{array}{l}\text { - L'harmonisation des mesures à caractère } \\
\text { normatif peut impliquer l'adoption } \\
\text { unilatérale, par une partie, d'un ensemble } \\
\text { de mesures et des guides d'une autre } \\
\text { partie; la négociation par les deux parties, } \\
\text { d'un ensemble commun de mesures et } \\
\text { guides (internationaux ou régionaux) ou un } \\
\text { rapprochement en direction des mesures et } \\
\text { guides internationaux ou régionaux en } \\
\text { vigueur. } \\
\text { - Dans le cas de la } 2^{\text {e }} \text { et de la } 3^{\text {e }} \text { option, } \\
\text { l'harmonisation implique souvent la } \\
\text { participation à des organismes } \\
\text { internationaux et/ou régionaux à activité } \\
\text { normative. } \\
\text { - L'harmonisation des règlements et normes } \\
\text { peut être intégrale (autrement dit s'effectuer } \\
\text { " produit par produit » et impliquer } \\
\text { l'harmonisation des caractéristiques } \\
\text { précises de chaque produit) ou se limiter } \\
\text { aux « caractéristiques essentielles » } \\
\text { (autrement dit le produit concerné doit } \\
\text { uniquement respecter des exigences } \\
\text { essentielles de sécurité, cf. marché unique } \\
\text { de l'UE). }\end{array}$ & $\begin{array}{l}\text { - Les entreprises doivent seulement } \\
\text { se conformer à un unique } \\
\text { ensemble de règlements, normes } \\
\text { et procédures d'évaluation de la } \\
\text { conformité. En outre, } \\
\text { l'harmonisation renforce la } \\
\text { compatibilité entre les produits } \\
\text { importés et les biens produits dans } \\
\text { le pays, ce qui stimule encore les } \\
\text { échanges internationaux. }\end{array}$ \\
\hline
\end{tabular}

6. Pour une analyse détaillée des différentes options d'action publique, voir Chen et Mattoo, 2004, Baller, 2007, Hoekman et Kostecki, 2001 et CE, 2001.

7. Les explications figurant dans le Tableau 2 sont purement indicatives et ne constituent pas des définitions universellement admises. 


\begin{tabular}{|c|c|c|}
\hline Principe essentiel & Portée & $\begin{array}{l}\text { Principaux avantages pour les } \\
\text { négociants des pays parties à } \\
\text { l'accord }\end{array}$ \\
\hline $\begin{array}{l}\text { Reconnaissance mutuelle } \\
\text { ou équivalence des } \\
\text { règlements techniques et } \\
\text { normes }\end{array}$ & $\begin{array}{l}\text { - Reconnaissance par une partie, comme } \\
\text { équivalents, des règlements techniques et } \\
\text { normes appliqués par une autre partie, } \\
\text { lorsque leurs spécifications techniques } \\
\text { diffèrent des leurs, dès lors que les parties } \\
\text { reconnaissent qu'ils remplissent les mêmes } \\
\text { objectifs réglementaires. (On entend par } \\
\text { « équivalence » la reconnaissance } \\
\text { unilatérale et par « reconnaissance } \\
\text { mutuelle » une reconnaissance } \\
\text { réciproque). } \\
\text { - La reconnaissance mutuelle accorde aux } \\
\text { produits conformes à l'un quelconque des } \\
\text { règlements et normes des autres parties un } \\
\text { accès illimité aux marchés de ces parties. }\end{array}$ & $\begin{array}{l}\text { - Les entreprises peuvent avoir } \\
\text { accès aux marchés des autres } \\
\text { parties sans avoir à se conformer à } \\
\text { leurs règlements et normes. En } \\
\text { respectant seulement un ensemble } \\
\text { donné de règlements et normes, } \\
\text { elles peuvent s'implanter sur tous } \\
\text { les marchés de l'autre partie. }\end{array}$ \\
\hline $\begin{array}{l}\text { Reconnaissance } \\
\text { (mutuelle) des résultats de } \\
\text { l'évaluation de la } \\
\text { conformité }\end{array}$ & $\begin{array}{l}\text { - Les autorités réglementaires des parties } \\
\text { reconnaissent la validité des rapports } \\
\text { d'essai ou les certificats délivrés par les } \\
\text { organismes d'évaluation de la conformité } \\
\text { des autres parties. (En l'absence de } \\
\text { reconnaissance des règlements techniques, } \\
\text { les produits sont évalués par rapport aux } \\
\text { règlements du pays importateur). } \\
\text { - Il existe des mécanismes très variés pour } \\
\text { faciliter l'acceptation de tous ou une partie } \\
\text { des résultats de l'évaluation de la } \\
\text { conformité d'une autre partie, notamment: } \\
\text { - L'utilisation de la déclaration de conformité } \\
\text { du fournisseur (le fournisseur fait de lui- } \\
\text { même une déclaration de conformité, } \\
\text { s'épargnant ainsi les coûts d'une évaluation } \\
\text { par un tiers)', } \\
\text { - Les accords volontaires conclus entre les } \\
\text { organismes d'évaluation de la conformité } \\
\text { applicables sur le territoire de chaque } \\
\text { partie à l'accord (autrement dit, les accords } \\
\text { « techniques » de reconnaissance } \\
\text { mutuelle), } \\
\text { - Les accords d'acceptation mutuelle des } \\
\text { résultats des procédures d'évaluation de la } \\
\text { conformité réalisées par les organismes } \\
\text { d'évaluation de la conformité des autres } \\
\text { parties (autrement dit, les accords } \\
\text { obligatoires « gouvernementaux » de } \\
\text { reconnaissance mutuelle) } \\
\text { les organismes d'évaluation de la } \\
\text { conformité. } \\
\text { d'organismes d'évaluation de la conformité. }\end{array}$ & $\begin{array}{l}\text { Les entreprises ne testent et ne } \\
\text { certifient pas leurs produits } \\
\text { plusieurs fois. Les essais et } \\
\text { certifications ont lieu avant } \\
\text { l'exportation des produits, qui } \\
\text { peuvent donc être directement } \\
\text { introduits sur les marchés des } \\
\text { autres parties sans avoir à être } \\
\text { soumis à de nouvelles procédures } \\
\text { d'évaluation de leur conformité. }\end{array}$ \\
\hline
\end{tabular}




\begin{tabular}{|c|c|c|}
\hline Principe essentiel & Portée & $\begin{array}{c}\text { Principaux avantages pour les } \\
\text { négociants des pays parties à } \\
\text { l'accord }\end{array}$ \\
\hline Transparence & $\begin{array}{l}\text { - Notification des règlements techniques, } \\
\text { normes et procédures d'évaluation de la } \\
\text { conformité avant leur adoption (pour } \\
\text { permettre aux autres parties ou aux } \\
\text { négociants de présenter leurs } \\
\text { observations). } \\
\text { - Publication des règlements techniques, } \\
\text { normes et procédures d'évaluation de la } \\
\text { conformité avant leur adoption. } \\
\text { - Mise en place de points d'information à } \\
\text { nationaux/régionaux à l'intention des } \\
\text { négociants. } \\
\text { - Possibilité de demander à la partie } \\
\text { notifiante d'expliquer les raisons pour } \\
\text { lesquelles elle n'utilise pas les normes } \\
\text { internationales, lorsqu'elles existent. } \\
\text { - Participation de personnes morales d'autres } \\
\text { parties à l'élaboration des règlements et } \\
\text { normes. }\end{array}$ & $\begin{array}{l}\text { - Les entreprises sont tenues } \\
\text { informées des règlements, normes } \\
\text { et procédures d'évaluation de la } \\
\text { conformité qu'elles doivent } \\
\text { respecter pour s'implanter sur les } \\
\text { marchés des autres parties et, dans } \\
\text { certains cas, peuvent formuler des } \\
\text { observations sur les règlements et } \\
\text { normes des autres parties ou } \\
\text { participer à leur élaboration. }\end{array}$ \\
\hline
\end{tabular}

1. Un fournisseur peut choisir cette option s'il jouit sur le marché d'une réputation suffisamment bonne qui le dispense de faire confirmer par des tiers indépendants la conformité de ses produits. De telles déclarations peuvent cependant ne pas être appropriées lorsque les risques pour la santé, la sécurité ou l'environnement sont plus élevés. La déclaration faite par le fournisseur lui même ne l'exonère pas de la responsabilité qui lui incombe de respecter les règlements techniques concernés et doit généralement s'accompagner d'une surveillance effective après son entrée sur le marché.

Source : ECDPM (à paraître), Baller, 2007 et http://www.iso.org.

\subsection{L'Accord de l'OMC sur les obstacles techniques au commerce}

20. Le fait que les règlements techniques et les normes puissent constituer des obstacles au commerce a été officiellement admis pour la première fois à l'échelon multilatéral durant le cycle de Tokyo, avec la négociation de l'Accord sur les obstacles techniques au commerce entré en vigueur en 1980 également connu sous l'appellation de Code des Normes. Il s'agissait alors d'un accord plurilatéral à caractère volontaire, dont les obligations ne s'appliquaient qu'aux membres ayant choisi de le ratifier (Kotschwar, 2001).

21. L'Accord sur les OTC de l'OMC conclu depuis étend et renforce le champ d'application et la couverture des disciplines internationales relatives aux règlements techniques et aux normes et énonce un ensemble complet de principes gouvernant la réglementation des OTC $^{8}$. Du fait que l'OMC exige l'adhésion de tous ses membres à l'Accord, le nombre de pays liés par ses obligations est bien plus important que celui des pays qui avaient pris des engagements en vertu du Code des Normes (Kotschwar, 2001). L'Accord sur les OTC de l'OMC vise à assurer que les règlements techniques, normes et procédures d'évaluation de la conformité applicables aux produits et aux procédés de production ne créent pas d'obstacles non nécessaires au commerce, tout en reconnaissant le droit des différents pays à adopter les normes de produits et règlements qu'ils estiment appropriés. Les principes et dispositions essentiels de l'Accord sont exposés ci-dessous (cf. Encadré 2).

8. Au nombre des autres accords de l'OMC traitant des normes et règlements figurent l'Accord de l'OMC sur les Mesures Sanitaires et Phytosanitaires («Accord MSP ») et l'AGCS, qui comporte des dispositions relatives aux normes dans le domaine des services. 
TAD/TC/WP(2007)12/FINAL

\section{Encadré 2. Principes et dispositions essentiels de l'Accord de l'OMC sur les obstacles techniques au commerce $^{1}$}

Non-discrimination et traitement national : L'article 2.1 de l'Accord dispose que « les membres feront en sorte, pour ce qui concerne les règlements techniques, qu'il soit accordé aux produits importés en provenance du territoire de tout membre un traitement non moins favorable que celui qui est accordé aux produits similaires d'origine nationale et aux produits similaires originaires de tout autre pays ॥. Le même principe vaut pour les procédures d'évaluation de la conformité et les redevances et demandes de renseignements qui s'y rapportent qui ne sauraient induire de discrimination vis-à-vis des produits importés. Les membres doivent respecter «le caractère confidentiel des renseignements concernant les produits originaires du territoire d'autres membres, qui peuvent résulter de l'évaluation de la conformité ou être fournis à cette occasion, de la même façon que dans le cas des produits d'origine nationale » (Art, 5.2.4 et 5.2.5).

Suppression des obstacles non nécessaires au commerce: Lorsque les pouvoirs publics préparent un règlement technique en vue de réaliser un objectif donné d'action publique, ce règlement technique ne sera pas plus restrictif pour le commerce qu'il n'est nécessaire pour réaliser un objectif légitime. Selon l'Accord, dans tous les cas où cela sera approprié, les membres définiront les règlements techniques basés sur les prescriptions relatives au produit en fonction des propriétés d'emploi du produit plutôt que de sa conception ou de ses caractéristiques descriptives (Art. 2.8). L'obligation de supprimer les obstacles non nécessaires au commerce s'applique aussi aux procédures d'évaluation de la conformité. Ces procédures ne seront pas plus strictes et la durée des procédures ne sera plus longue qu'il n'est nécessaire pour évaluer la conformité d'un produit avec les lois et réglementations du pays.

Harmonisation des règlements techniques, normes et procédures d'évaluation de la conformité : L'Accord invite les États à utiliser les normes internationales existantes ou leurs éléments pertinents comme base des règlements techniques qu'ils élaborent et à utiliser les guides ou recommandations internationaux ou leurs éléments pertinents comme base de leurs procédures d'évaluation de la conformité. L'Accord autorise toutefois des exceptions lorsque les normes, guides et recommandations internationaux « seraient inefficaces ou inappropriés pour réaliser les objectifs légitimes recherchés » par un pays (Art. 2.4 et 5.4). De plus, les États doivent participer, « dans les limites de leurs ressources, à l'élaboration, par les organismes internationaux à activité normative compétents, de normes internationales concernant les produits pour lesquels ils ont adopté, ou prévoient d'adopter, des règlements techniques " et à l'élaboration de guides ou recommandations concernant les procédures d'évaluation de la conformité.

Acceptation des règlements techniques comme équivalents : Parallèlement à l'harmonisation, l'Accord encourage les membres à " accepter comme équivalents les règlements techniques des autres membres à condition que ces règlements remplissent de manière adéquate les objectifs de leurs propres règlements » (Art. 2.7).

Reconnaissance mutuelle de l'évaluation de la conformité : En outre, l'Accord encourage les membres à reconnaître, "chaque fois que cela sera possible, les résultats des procédures d'évaluation de la conformité d'autres membres » destinées à évaluer si un produit est conforme aux règlements techniques dont le respect est obligatoire. En l'absence d'une telle reconnaissance, il pourrait être nécessaire de faire tester les produits deux fois, une fois par le pays exportateur et une autre fois par le pays importateur. L'Accord encourage aussi les membres « à bien vouloir se prêter à des négociations en vue de la conclusion d'accords de reconnaissance mutuelle des résultats de leurs procédures d'évaluation de la conformité » (" accords de reconnaissance mutuelle " ou ARM, Art. 6.3). Les ARM nécessitent cependant la confiance dans la compétence des organismes et procédures d'évaluation de la conformité des autres membres. L'Accord de l'OMC reconnaît par conséquent que des consultations préalables pourront être nécessaires pour arriver à un accord mutuellement satisfaisant au sujet de la compétence des organismes d'évaluation de la conformité concernés (Art. 6.1).

Transparence : Pour contribuer à assurer la transparence, chaque membre de l'OMC, fera en sorte qu'il existe un point d'information national (Art. 10) et de notifier au Secrétariat de l'OMC ses règlements techniques et procédures d'évaluation de la conformité, d'en discuter et les faire paraître, chaque fois qu'ils n'existaient pas ou que leur teneur n'est pas conforme aux normes internationales, recommandations ou guides internationaux existants, ou qu'ils peuvent avoir un effet notable sur le commerce d'autres membres, (Art. 2.9 et 5.6). Les membres feront paraître assez tôt une publication à ce sujet et les notifieront aux autres membres, par l'intermédiaire du Secrétariat de l'OMC, en indiquant brièvement l'objectif et la raison d'être du nouveau règlement ou des procédures d'évaluation de la conformité projetées. Enfin, ils ménageront « un délai raisonnable » aux autres membres pour leur permettre de présenter leurs observations sur les règlements techniques projetés avant leur entrée en vigueur, délai qui devra être de « 60 jours au moins », conformément à la recommandation du Comité OTC. Le Code de pratiques applicable aux normes (dont le respect est volontaire) dispose expressément que l'organisme à activité normative ménagera une période de 60 jours au moins aux parties intéressées pour présenter leurs observations au sujet du projet de norme ${ }^{2}$. 
Assistance technique : L'Accord invite les membres à fournir une assistance technique aux autres membres (Art. 11) qui es notifieront, par exemple, l'élaboration de règlements techniques, la création d'organismes nationaux à activité normative, la participation aux travaux des organismes internationaux à activité normative et la fourniture ou le renforcement des équipements et capacités nécessaires aux essais et à la certification.

Traitement spécial et différencié : Dans l'élaboration et l'application des règlements techniques, des normes et des procédures d'évaluation de la conformité, les membres tiendront compte des besoins spéciaux du développement, des finances et du commerce des pays en développement. II est en outre reconnu que l'on ne saurait attendre des pays en développement qu'ils utilisent des normes internationales qui ne sont pas appropriées aux besoins de leur développement, de leurs finances et de leur commerce. Enfin, le Comité des obstacles techniques au commerce est habilité à faire bénéficier les pays en développement d'exceptions spécifiées et limitées dans le temps, totales ou partielles aux obligations résultant de l'Accord (Art.12).

Mise en œuvre de l'Accord et règlement des différends : Le Comité des obstacles techniques au commerce de I'OMC est la principale enceinte donnant aux membres la possibilité de procéder à des consultations sur toute question concernant le fonctionnement de l'Accord et de discuter des préoccupations relatives aux règlements et à leur mise en œuvre (Art. 13). Pour résoudre les problèmes relatifs aux OTC survenant entre les pays, l'Accord sur les OTC renvoie expressément à l'Organe de règlement des différends de l'OMC en ce qui concerne la tenue des consultations et le règlement des différends (Art. 14).

Divers : L'Accord définit en outre un « Code de pratique » pour l'élaboration, l'adoption et l'application des normes dont le respect est volontaire, qui est ouvert à l'acceptation de tout organisme à activité normative du secteur privé comme du secteur public (cf. Annexe 3 de l'Accord).

1. Toutes ces obligations ne s'appliquent qu'au gouvernement central.

2. «Le Comité recommande de ménager un délai normal de 60 jours au moins pour la présentation des observations. Tout membre qui est en mesure d'accorder un délai supérieur à 60 jours est encouragé à le faire ". Comité des obstacles techniques au commerce - Décisions et recommandations adoptées par le Comité depuis le $1^{\text {er }}$ janvier 1995. Note du Secrétariat - Révision, G/TBT/1/Rev.8, Organisation mondiale du commerce, 23 mai 2002.

Source : Accord de l'OMC sur les obstacles techniques au commerce, OMC - Portail - Les Accords commerciaux régionaux et Comité des obstacles techniques au commerce de l'OMC. 


\section{RÈGLES RÉGIONALES EN MATIÈRE D'OTC : CONVERGENCE OU DIVERGENCE PAR RAPPORT AU SYSTÈME COMMERCIAL MULTILATÉRAL ?}

\subsection{Introduction}

22. Parallèlement aux engagements pris conformément à l'Accord sur les OTC de l'OMC, la quasitotalité des membres de l'OMC ont conclu des accords préférentiels (cf. Portail de l'OMC sur les Accords commerciaux régionaux $)^{9}$. Ces dix dernières années, le nombre d'accords commerciaux bilatéraux, régionaux et plurilatéraux (conjointement appelés " $\mathrm{ACR} »)$ a considérablement augmenté et cette tendance devrait se poursuivre (cf. Graphique 1) ${ }^{10}$. Si l'on en croit certains experts, en tenant compte des ACR actuellement en cours de négociation ou de ratification, leur nombre devrait atteindre les 300 en 2008 (Crawford et Fiorentino, 2005).

Graphique 1. Nombre d'ACR notifiés en vigueur, au mois de mars 2007

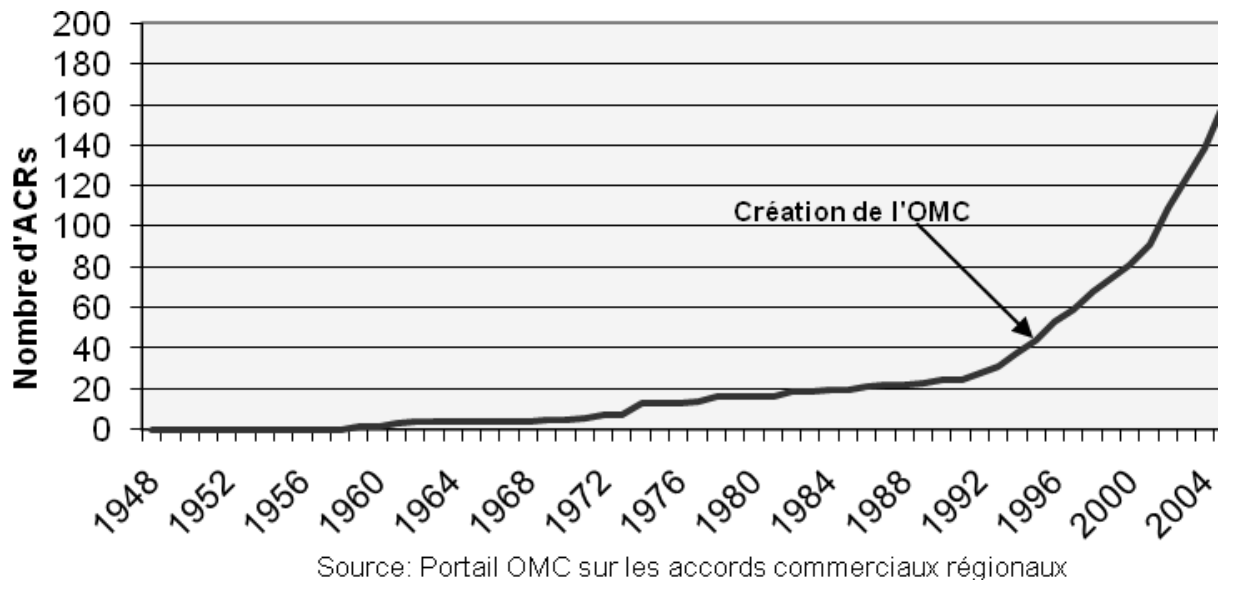

23. Compte tenu du nombre croissant d'ACR, ces accords vont probablement contribuer de plus en plus à instaurer une discipline en matière d'OTC, mais ils peuvent aussi dupliquer (ou contredire) les efforts multilatéraux accomplis. Une étude récente des Secrétariats de l'OMC et de la Banque interaméricaine de développement (BID) constate par exemple que sur 70 ACR examinés - représentant environ $1 / 3$ du total des ACR notifiés à l'OMC - 58, soit plus de $80 \%$ d'entre eux, contiennent des dispositions relatives aux OTC (Piermartini et Budetta, 2006).

24. Pourtant, l'augmentation du nombre d'ACR a eu pour effet l'adhésion des différents pays à plusieurs accords à la fois. Chaque ACR aspirant à mettre en place son propre mini-régime commercial, la

9. Les Membres de l'OMC sont en droit de conclure de tels accords à des conditions particulières. L'Article XXIV du GATT prévoit des dispositions sur la constitution et du fonctionnement d'unions douanières et de zones de libre-échange concernant le commerce des marchandises ; la clause d'habilitation fait référence aux accords commerciaux préférentiels dans le domaine du commerce des marchandises entre pays Membres en développement et l'Article V de l'AGCS régit la conclusion d'ACR dans le domaine du commerce des services.

10. Le nombre d'ACR est passé de 53 accords notifiés en 1996 à 178 en 2006, soit une hausse de $236 \%$. 
coexistence dans un même pays de règles commerciales dissemblables s'appliquant aux différents partenaires des ACR est devenue une réalité courante. Ce phénomène peut entraver les flux commerciaux, ne serait-ce que par les coûts qui en découlent pour les négociants tenus de se conformer à plusieurs ensembles de règles commerciales, comme par exemple des normes de produits, des règlements techniques et des procédures d'évaluation de la conformité différents. La prolifération d'ACR accroît de ce fait le risque d'incohérence des normes et procédures entre les $A C R$ eux-mêmes, ainsi qu'entre les $A C R$ et le cadre multilatéral (Banque mondiale, 2005). Outre leur adhésion à des ACR, certains pays s'impliquent aussi dans une coopération réglementaire par le biais d'accords de reconnaissance mutuelle (ARM) en vue d'éviter la duplication des procédures d'essai et de certification des produits.

25. Pour déterminer s'il existe des incohérences entre les règles régionales et les règles multilatérales relatives aux OTC, ce chapitre examine les dispositions relatives aux OTC d'un échantillon d'ACR à l'aune des 7 catégories répertoriées au Tableau 1 et relève les cas où ces dispositions vont au-delà des règles de l'OMC (partie 3.2). Il identifie ensuite les facteurs susceptibles d'influer sur l'approche retenue en vue de réduire les OTC (partie 3.3) et évalue la convergence (ou la divergence) de chaque ACR par rapport au système commercial multilatéral (partie 3.4).

\subsection{Vue d'ensemble des dispositions relatives aux OTC d'un échantillon d'accords commerciaux ${ }^{11}$}

\subsubsection{Référence à l'Accord sur les OTC de l'OMC}

26. Les dispositions relatives aux OTC contenues dans les ACR conclus avant la création de l'OMC rappellent généralement les objectifs essentiels de l'Accord sur les OTC de l'OMC, soulignant que les OTC ont pour objectifs légitimes la protection de la santé publique, de la sécurité et de l'environnement public. Certains chapitres sur les OTC renvoient en outre à des objectifs ayant une plus grande portée, comme la promotion de l'investissement entre les parties (comme le prévoit l'accord d'association entre les Communautés européennes et le Chili), l'amélioration des conditions pour les entreprises (accord Chine-Singapour) et de la qualité et de l'efficacité de la production (marché commun des Caraïbes ou CARICOM). Le cas échéant, les dispositions relatives aux OTC visent généralement à renforcer et à faciliter le commerce par une mise en œuvre plus poussée de l'Accord sur les OTC de l'OMC. De plus, la plupart de ces ACR réaffirment les droits et obligations des parties en vertu de l'Accord sur les OTC de $1^{\prime} \mathrm{OMC}^{12}$. Ainsi, aux termes de l'accord de libre-échange entre le Maroc et les États-Unis, «les parties confirment les droits et obligations existants qu'elles ont les unes envers les autres en vertu de l'Accord sur les OTC » [de l'OMC] » (Article 7.2).

\subsubsection{Harmonisation des règlements techniques, normes et procédures d'évaluation de la conformité}

27. Près de la moitié des ACR examinés préconisent ou encouragent une harmonisation des règlements techniques, normes et procédures d'évaluation de la conformité. Ils incitent dans leur grande majorité à utiliser les normes et guides internationaux comme base à l'élaboration des règlements

11. Les ACR étudiés dans ce chapitre comprennent 24 accords examinés dans les études de cas, ainsi que des accords supplémentaires examinés par d'autres experts, comme Piermartini et Budetta, 2006, Kotschwar, 2001 et l'ECDPM (à paraître). La liste des ACR examinés est jointe en Annexe I et la synthèse des résultats provenant des études de cas en Annexe II.

12. Il ressort des études de cas que $86 \%$ des ACR en moyenne font référence à l'Accord sur les OTC de l'OMC (ses objectifs, règles et/ou dispositions), et 40\% en moyenne à des objectifs allant encore au-delà. De même, selon Piermartini et Budetta (2006), 50 \% des ACR examinés font référence à l'Accord sur les OTC de l'OMC et $36 \%$ d'entre eux réaffirment les droits et obligations mutuelles des parties en vertu de cet Accord. 
techniques, procédures d'évaluation de la conformité et normes, sauf lorsque les normes internationales sont inefficaces ou inappropriés pour réaliser les objectifs légitimes recherchés ${ }^{13}$. Ainsi l'ASEAN invite ses membres à harmoniser leurs normes de produits en les mettant au niveau des pratiques internationales (OCDE, 2005). De plus, certains ACR contiennent des dispositions prévoyant une consultation mutuelle en vue d'instaurer une plus grande communauté de vues sur les questions internationales concernant les OTC (par exemple l'ALADI, la CARICOM et le MERCOSUR, cf. Encadré 7, ci-dessous).

28. Parallèlement, près d'un tiers de tous les ACR examinés préconise une harmonisation par le biais de l'utilisation et de l'élaboration de règlements, normes et principes d'évaluation de la conformité régionaux ${ }^{14}$. En réalité, les ACR peuvent parfois promouvoir des normes et recommandations régionales parallèlement aux normes et recommandations internationales. Le Partenariat économique stratégique transpacifique conclu entre le Chili, Singapour, la Nouvelle-Zélande et le Brunei impose ainsi aux parties d'utiliser comme bases les normes internationales existantes pour élaborer leurs règlements techniques et procédures d'évaluation de la conformité connexes et leur recommande de s'appuyer sur les travaux régionaux de l'APEC sur les normes et la conformité et d'y prendre part (Art. 8). De même, la Communauté andine invite ses membres à se fonder sur les normes internationales (ou leurs éléments pertinents) pour élaborer leurs règlements techniques, «sauf lorsque les normes internationales ou leurs éléments pertinents sont un moyen inefficace ou inapproprié pour réaliser les objectifs légitimes recherchés ». Dans ce cas, les règlements techniques nationaux se fondent sur des règles de la Communauté andine infrarégionales, régionales et/ou nationales (Décision 562). De même, pour les normes dont le respect est volontaire, le Marché commun de l'Afrique de l'Est et de l'Afrique australe (COMESA) encourage l'instauration de normes régionales africaines lorsqu'il n'existe par de normes internationales ou que celles-ci semblent inappropriées.

29. Un certain nombre d'accords d'association économique de l'UE avec des pays candidats à l'adhésion (des pays d'Europe centrale et orientale par exemple) ou des «pays voisins » (comme ceux de l'Espace économique européen, d'Afrique du nord et d'Europe du Sud-Est) invite les parties à coopérer pour promouvoir l'utilisation des règles techniques de la Communauté et des normes, procédures d'essai et d'évaluation de la conformité européennes. Dans les faits, ces pays sont tenus d'adopter unilatéralement les règles de l'UE (comme le prévoit par exemple l'accord EUROMED conclu entre l'UE et le Maroc par exemple). L'UE souligne néanmoins que ses règlements techniques, normes et procédures d'évaluation de la conformité reposent sur des normes et guides internationaux, lorsqu'ils existent (Jenkinson dans OCDE, $2005)^{15}$. À cet égard, Shortall (2006) relève qu'à la fin 2006, près de $30 \%$ des normes européennes se fondaient sur des normes internationales.

13. Il ressort des études de cas que $47 \%$ des ACR imposent aux parties ou les encouragent à harmoniser leurs règlements, normes et procédures d'évaluation de la conformité. Selon Piermartini et Budetta (2006), $46 \%$ d'entre eux en moyenne comportent des dispositions de ce type. Ces deux catégories d'études soulignent que $60 \%$ à $70 \%$ environ des dispositions sur l'harmonisation incitent à utiliser les normes et guides internationaux.

14. Il ressort des études de cas que $34 \%$ des ACR en moyenne incitent à utiliser des règlements, normes et procédures d'évaluation de la conformité régionaux. Selon Piermartini et Budetta (2006), ils sont $45 \%$ en moyenne à contenir des dispositions de ce type.

15. La Commission européenne relève que le processus de normalisation internationale peut être péniblement long. En conséquence, l'UE, qui est le plus important participant à ce processus et qui s'y implique fortement, a relativement utiliser les nouvelles normes internationales pour élaborer sa législation technique et permettre l'ouverture des échanges. Source : http://trade-info.cec.eu.int/tbt/index.cfm 


\subsubsection{Acceptation des règlements techniques comme équivalents}

30. Contrairement à l'exigence courante d'harmonisation des règlements techniques et des normes, un nombre plus réduit d'ACR obligent ou encouragent leurs membres à envisager comme équivalents les règlements techniques et normes d'autres parties. Il est intéressant de noter que près de $40 \%$ de ces ACR imposent aux parties de donner une explication lorsqu'elles n'appliquent par le principe de l'équivalence des règlements techniques des autres parties à l'accord, et vont donc au-delà des règles de l'OMC (comme c'est le cas pour de nombreux accords conclus par les États-Unis) ${ }^{16}$. L'accord de libre-échange conclu entre l'Amérique centrale, la République dominicaine et les États-Unis (Accord de libre-échange de l'Amérique centrale ou ALECA) note par exemple que lorsqu'une partie prévoit que les règlements techniques étrangers peuvent être acceptés comme équivalant à l'une de ses propres spécifications techniques et qu'elle n'accepte pas comme équivalent à ce règlement technique celui d'une autre partie, la partie en question doit, à la demande de cette autre partie, expliquer les raisons de sa décision. Lorsqu'une partie ne prévoit pas que les règlements techniques étrangers peuvent être acceptés comme équivalant aux siens, elle peut, à la demande d'une autre partie, expliquer pour quelles raisons elle n'accepte pas comme équivalents les règlements techniques des autres parties. » (Art. 7.6).

31. Enfin, une petite fraction des ACR examinés ne précise pas expressément quelle approche (harmonisation ou équivalence des règlements techniques et normes) doit être adoptée par les parties. Certains ACR conclus par le Chili avec ses voisins d'Amérique latine (par exemple avec le Mexique, le Salvador et le Costa Rica) imposent ainsi aux parties d'assurer la "compatibilité » des mesures relatives aux normes. De même, certains ACR (par exemple les accords Maroc-AELE, Chili-AELE, MarocTurquie) encouragent « une plus grande coopération » en matière de règlements techniques, de normes et d'évaluation de la conformité, sans préciser quelle forme cette coopération doit prendre.

\subsubsection{Reconnaissance (mutuelle) des évaluations de la conformité}

32. Les dispositions de reconnaissance mutuelle des règlements techniques et normes d'autres parties ne s'appliquent pour l'instant que dans l'UE (Baller, 2007, voir Encadré 8). Cela étant, près de $70 \%$ des ACR examinés encouragent les parties à reconnaître (mutuellement) les résultats de leurs procédures d'évaluation de la conformité. Près d'un quart d'entre eux vont au-delà de la règle de l'OMC à cet égard, en précisant que lorsqu'une partie n'accepte pas les résultats de la procédure d'évaluation de la conformité conduite sur le territoire d'une autre parie, cette partie doit expliquer, à la demande des autres parties, les raisons de son refus ${ }^{17}$.

33. De plus, certains de ces accords (comme le Partenariat économique stratégique transpacifique et plusieurs accords auxquels les États-Unis sont parties) incitent chaque partie à reconnaître les organismes d'évaluation de la conformité situés sur le territoire de l'autre partie «à des conditions non moins

16. Il ressort des études de cas que $33 \%$ en moyenne de l'ensemble des ACR examinés encouragent l'acceptation des règlements techniques (et des normes) comme équivalents. Sur l'ensemble des ACR examinés, $33 \%$ imposent aux parties d'expliquer les raisons pour lesquelles elles n'acceptent pas l'équivalence des règlements. Piermartini et Budetta (2006) montre que $26 \%$ des ACR encouragent l'équivalence des règlements techniques, et que $47 \%$ d'entre eux imposent aux parties d'expliquer leurs raisons, en cas de refus.

17. Il ressort des études de cas que $77 \%$ des $\mathrm{ACR}$, en moyenne, contiennent des dispositions de reconnaissance mutuelle des résultats des évaluations de la conformité, $34 \%$ d'entre eux allant au-delà des dispositions de l'OMC. Selon Piermartini et Budetta (2006), 67 \% des ACR examinés comportent de telles dispositions et sur ce nombre, $15 \%$ sont plus exigeants que les règles de l'OMC. Selon Kotschwar (2001), la « quasi-totalité » des ACR examinés contiennent des dispositions prévoyant la reconnaissance mutuelle des résultats des procédures d'évaluation de la conformité. 
favorables que celles qui sont accordées aux organismes d'évaluation de la conformité situés sur son territoire » et de fournir des explications, sur demande, si elle refuse d'accréditer, d'approuver, d'agréer ou de reconnaître un tel organisme. L'accord de libre-échange entre les États-Unis et le Chili prévoit ainsi que " [...] Si une partie accrédite, approuve, agrée ou reconnaît de toute autre façon un organisme évaluant la conformité avec un règlement technique ou une norme donné sur son territoire et refuse d'accréditer, d'approuver, d'agréer ou de reconnaître d'une autre façon, un organisme évaluant la conformité avec ce règlement technique ou cette norme sur le territoire de l'autre partie, celle-ci devra expliquer, si on lui en fait la demande, les raisons de son refus " (Accord États-Unis-Chili, Art. 7.6). Ces dispositions vont clairement au-delà des engagements de l'OMC qui encouragent seulement les membres à permettre la participation d'organismes d'évaluation de la conformité situés sur le territoire d'autres membres (Art. 6.4, l'Accord sur les OTC de l'OMC). De plus, conformément à la décision du Comité OTC de l'OMC de 2000, plusieurs ACR (comme l'ALECA) encouragent la reconnaissance des résultats des évaluations de la conformité par l'intermédiaire de mécanismes très variés que sont notamment la reconnaissance des déclarations de conformité des fournisseurs - n'exigeant pas qu'un tiers vérifie si un produit est conforme aux règlements techniques et aux normes en vigueur - et la conclusion d'accords, dont le respect est volontaire, entre les organismes d'évaluation de la conformité de chaque partie.

34. Enfin, dans le cas de plusieurs ACR examinés, les parties ont aussi conclu des Accords de reconnaissance mutuelle (ARM) avec d'autres parties. Par l'intermédiaire de ces accords, les essais et la certification des produits ont lieu avant leur exportation et les produits peuvent être introduits directement dans le pays importateurs sans avoir besoin d'y être soumis à des procédures similaires d'évaluation de la conformité (cf. Encadré 3). Les ARM sont souvent conclus indépendamment de l'ACR général et s'appliquent secteur par secteur (par exemple pour les équipements de télécommunications, électriques et électroniques ou le matériel médical) ${ }^{18}$. Ils sont souvent jugés moins intrusifs et moins onéreux que l'harmonisation directe des règlements, normes et procédures d'évaluation de la conformité, puisqu'ils n'imposent pas aux pays de modifier leurs règlements nationaux (Baller, 2007 and EC, 2001). Ils supposent pourtant une forte confiance mutuelle dans l'efficacité des procédures d'évaluation de la conformité et les compétences des organismes d'évaluation de la conformité des parties à l'accord, ainsi que le tenue de négociations et la mise en œuvre des ressources administratives et capacités nécessaires (APEC, 2006). Pour l'heure, la plupart des ARM gouvernementaux ont de ce fait été conclus et effectivement mis en œuvre entre pays développés (à revenu élevé et moyen supérieur) dotés de l'infrastructure d'évaluation de la conformité nécessaire (comme les accords conclus entre l'UE et d'autres pays de l'OCDE, entre les pays développés de l'ASEAN et entre les pays développés de l'APEC. Cf. Étude de cas sur Singapour). Un certain nombre d'ACR conclus entre pays développés et en développement encouragent cependant la conclusion d'ARM distincts et contiennent des dispositions relatives à la fourniture d'une assistance technique aux parties les plus pauvres, pour renforcer leurs capacités juridiques, réglementaires et leur infrastructure dans cette optique (accord de libre-échange conclu entre les États-Unis et le Maroc, ASEAN et APEC).

18. L’UE adopte généralement une approche multisectorielle vis-à-vis des ARM (Baller, 2007). 


\section{Encadré 3. Types d'accords de reconnaissance mutuelle (ARM) relatifs à l'évaluation de la conformité}

Les experts établissent une distinction entre ARM techniques et ARM gouvernementaux. Les ARM techniques sont conclus par des organismes techniques (laboratoires d'essai, organismes d'inspection, de certification, d'accréditation). Leur respect est volontaire, mais ces accords facilitent souvent la conclusion d'agréments à l'échelon gouvernemental. Les ARM gouvernementaux sont souvent conclus pour certains secteurs productifs donnés sous la tutelle des pouvoirs publics.

Baller (2007) distingue 3 catégories différentes d'ARM gouvernementaux :

- L'harmonisation complète des procédures d'évaluation de la conformité comme dans le cas du marché unique de l'Union européenne.

- L'équivalence de la conformité (reconnaissance unilatérale de la conformité), comme dans le cas de l'ARM Trans-Tasman entre l'Australie et la Nouvelle-Zélande.

- La pleine reconnaissance des évaluations de la conformité : la majorité des ARM gouvernementaux appartient à cette catégorie qui se fonde sur la pleine reconnaissance des résultats des évaluations de la conformité. Cela suppose que les partenaires essaient et certifient les produits conformément aux normes et aux obligations du pays importateur ; un certificat attestant de la conformité complète des produits à ces obligations est ensuite délivré par le pays exportateur et doit être accepté par le pays importateur.

Les ARM ne prévoient pas, en règle générale, l'équivalence des règlements techniques. L'ARM conclu entre l'UE et les États-Unis sur les équipements marins se distingue à cet égard puisqu'il renforce la coopération en allant au-delà de la simple acceptation des résultats des évaluations de la conformité et des certificats, du fait qu'il reconnaît l'équivalence des règlements techniques respectifs de ses membres. (Ces ARM sont parfois appelés ARM « étendus » de l'UE).

Source : Baller, 2007, APEC, 2006 et Poncin, 2002.

\subsubsection{Transparence}

35. La majorité des ACR examinés contient des dispositions sur la transparence invitant instamment les membres à se notifier mutuellement les nouveaux règlements techniques et procédures d'évaluation de la conformité ou les modifications des règlements et procédures existants lorsqu'ils ne sont pas conformes aux normes internationales ou sont susceptibles d'avoir un effet notable sur le commerce ${ }^{19}$. Près d'un tiers des ACR examinés impose la mise en place d'un dispositif d'échange d'informations (par exemple de points d'informations régionaux) concernant les règlements techniques et procédures d'évaluation de la conformité dans le cadre de l'ACR. Une minorité d'entre eux (moins de $10 \%$ ) va au-delà des règles de l'OMC, en précisant que leurs membres doivent ménager aux autres parties, pour la présentation de leurs observations, un délai supérieur à 60 jours avant l'adoption ou la modification d'un règlement technique ou d'une procédure d'évaluation de la conformitée ${ }^{20}$. La Communauté andine impose ainsi aux pays membres de notifier aux autres parties le projet de règlement technique qu'ils prévoient d'adopter, au moins quatre-vingt-dix jours calendaires avant sa publication officielle, pour permettre la réception et la prise en compte de leurs observations (Décision 562).

19. Il ressort des études cas que $80 \%$ des ACR en moyenne contiennent des engagements en matière de transparence. Piermartini et Budetta (2006) fait état de $52 \%$ et Kotschwar (2001) de $66 \%$ d'entre eux.

20. D'après les études de cas, si $60 \%$ des ARC en moyenne ménagent un délai de réception des observations, aucun d'entre eux ne va cependant au-delà de la recommandation de l'OMC en fixant un délai supérieur à 60 jours. Selon Piermartini et Budetta (2006), $16 \%$ des ACR vont au-delà des règles de l'OMC à cet égard. 


\subsubsection{Mise en application et règlement des différends}

\section{Institutions}

36. La majorité des ACR examinés préconise la mise en place d'un comité, organisme ou réseau conjoint à cet égard ${ }^{21}$. Ces institutions suivent généralement la mise en œuvre des dispositions de l'accord, élaborent des propositions d'améliorations futures des dispositions relatives aux OTC, facilitent les exchanges d'informations et servent d'enceintes consultatives pour les questions relatives aux OTC. De plus, elles coordonnent parfois des initiatives en vue d'une harmonisation et gèrent les dispositifs visant à renforcer la coopération entre les parties, comme les ARM (ainsi que le fait, par exemple, le Comité consultatif de l'ASEAN sur les normes et la qualité, cf. Encadré 4), conseillent les parties ou les aident à fournir une assistance technique (comme le fait le Comité spécial Chili-UE sur les règlements techniques, les normes et l'évaluation de la conformité). D'autres accords imposent par ailleurs aux parties de se consulter mutuellement et de suivre la mise en œuvre du chapitre sur les OTC qui y est contenu en désignant des "coordinateurs » au sein de chaque partie (comme le prévoient l'accord conclu entre Singapour et le Panama et certains accords bilatéraux auxquels les États-Unis sont parties). D'autres ACR mettent en place des comités OTC sectoriels, soit parce qu'a été retenue une approche sectorielle en matière d'OTC soit parce que la portée de l'accord est limitée (l'accord conclu entre le Chili et le Canada contient ainsi des dispositions relatives aux OTC qui ne s'appliquent qu'au secteur des télécommunications).

37. Enfin, lorsqu'un comité chargé des affaires relatives aux normes n'a pas été mis en place et que des coordinateurs n'ont pas été désignés, certains accords (comme l'accord EUROMED entre l'UE et le Maroc) prévoient une coopération entre les parties pour assurer un soutien en matière d'organisation en vue de favoriser la mise en place d'organismes et de réseaux régionaux.

\section{Encadré 4. Comité consultatif sur les normes et la qualité de l'ASEAN (ACCSQ)}

Le comité consultatif sur les normes et la qualité de l'ASEAN (ACCSQ) s'est efforcé d'harmoniser les normes nationales avec les normes internationales (ISO, CEI et UIT par exemple) et met en œuvre des accords de reconnaissance mutuelle des évaluations de la conformité pour réaliser l'objectif ultime : « une seule norme, un seul essai et une acceptation universelle ».

L'ACCSQ met en œuvre ou suit la mise en œuvre de l'accord par ses groupes de travail et ses groupes produits dans plusieurs domaines:

- L'harmonisation des normes : Le Comité a supervisé l'harmonisation de normes applicables à 20 produits prioritaires (qui a été achevée en 2003), identifie et agrée de nouveaux domaines d'harmonisation des normes et de coopération pour les besoins des activités internationales de normalisation. En novembre 2005, 140 normes avaient été harmonisées sur la base des normes internationales et les instituts nationaux de normalisation de nombreux pays de l'ASEAN ont pris part aux travaux d'organismes internationaux à activité normative.

- L'harmonisation des règlements techniques et des évaluations de la conformité : le Comité a œuvré à l'harmonisation des règlements techniques et des régimes d'évaluation de la conformité de quatre secteurs donnés (équipements électriques et électroniques, cosmétiques, produits pharmaceutiques et préparations alimentaires industrielles). L'ASEAN entend mettre en place un régime réglementaire entièrement harmonisé pour les équipements électriques et électroniques d'ici 2010. Des groupes de travail ont également été constitués pour les secteurs de l'automobile, des médecines traditionnelles et des suppléments de santé, du bois, du caoutchouc et des appareils médicaux.

21. Il ressort des études cas que $80 \%$ des ACR examinés comportent de telles obligations. Selon Piermartini et Budetta (2006), 62\% d'ARC examinés contiennent de tels engagements. 
- L'élaboration et l'application d'ARM sectoriels : le Comité a donné l'élan à la conclusion, en 1998, de l'Accord cadre de l'ASEAN relatif aux accords de reconnaissance mutuelle énonçant les principes élémentaires pour l'élaboration et la conclusion d'ARM sectoriels. Depuis, trois ARM sectoriels ont été conclus et des travaux sont en cours dans deux autres secteurs: pour les équipements de télécommunications (signé en 2001), pour les équipements électriques et électroniques (signé en 2002, qui devait prendre pleinement effet en 2005), les cosmétiques (signé en 2003 qui devrait prendre pleinement effet en 2008), les produits pharmaceutiques (en cours d'élaboration) et les préparations alimentaires industrielles (en cours d'élaboration).

- L'accréditation et l'évaluation de la conformité : le Comité examine les exigences en matière de reconnaissance des organismes d'accréditation internationaux et régionaux et aide les pays membres à s'y conformer, renforce les compétences des laboratoires et des organismes de certification des produits et des systèmes de qualité des pays de l'ASEAN.

- La métrologie légale : le Comité participe à l'harmonisation de la législation et de l'administration dans le domaine de la métrologie légale.

- La transparence : citons par exemple le Bulletin de l'ASEAN sur les normes et la qualité, qui est publié régulièrement en vue de diffuser des informations sur les normes, les règlements techniques et les procédures d'évaluation de la conformité et d'en renforcer la transparence dans les pays de l'ASEAN.

- Renforcement de l'infrastructure technique : notamment à destination des pays en développement.

Les experts reconnaissent que l'ACCSQ a considérablement progressé ces dernières années sur la voie d'une coopération régionale en matière de normes et d'évaluation de la conformité.

Source : http://www.aseansec.org, ASEAN, 2003 et OCDE, 2005.

38. Dans l'intention de renforcer l'intégration et l'harmonisation, les unions douanières et les accords visant en dernier ressort à instaurer un marché commun prévoient souvent la mise en place d'organismes de normalisation régionaux (marché unique de l'UE, ASEAN et APEC). Le COMESA, a ainsi institué un organisme commun, l'Organisation régionale africaine de normalisation (ORAN), à laquelle sont soumises toutes les questions relatives aux OTC. Cependant, les contraintes financières et le faible taux d'acceptation par ses membres ont limité les progrès des travaux de l'Organisation. Une étude interne montre en effet qu'elle bénéficie d'un faible soutien public de la part de ses membres et que les intéressés la jugent sévèrement. En 2003, l'Organisation avait publié 733 normes de produits régionales africaines (s'appuyant principalement sur les normes internationales), qui n'ont été pour la majorité d'entre elles que partiellement adoptées (ORAN, 2003). De même, l'organisme de normalisation de la CARICOM, l'Organisation régionale sur les normes et la qualité de la CARICOM (CROSQ), parait ne pas avoir le personnel suffisant pour promouvoir les efforts de normalisation régionaux ${ }^{22}$.

\section{Règlement des différends}

39. Plus de la moitié des ACR examinés comportent des dispositions sur la résolution des différends relatifs aux OTC survenant entre leurs membres. La plupart d'entre elles invitent à organiser des discussions et des consultations au sein des comités régionaux en cas de désaccord ${ }^{23}$. Certains accords (comme l'ALADI et les accords conclus entre le Mexique et le Nicaragua, le Mexique et Costa Rica et le Mexique et la Bolivie) envisagent en outre le recours à des sous-groupes techniques ou à des groupes de travail ad hoc pouvant formuler des recommandations non contraignantes.

22. Voir http://www.crosq.org/.

23. Il ressort des études de cas que $87 \%$ des ACR examinés précisent les procédures de règlement des différends applicables. Selon Piermartini et Budetta (2006), quelque $50 \%$ des ACR comportent de telles dispositions. Dans tous les cas, les dispositions en question invitent les parties à procéder à des consultations. 
40. Enfin, un nombre plutôt limité d'ACR contenant des dispositions relatives aux OTC prévoit un dispositif plus formel de résolution des différends se rapportant aux normes (principalement par voie d'arbitrage), allant de ce fait au-delà de l'Accord sur les OTC de l'OMC. Dans le cas de la Communauté andine, un conseil a ainsi été établi qui peut ordonner la révocation d'un règlement technique, d'une norme ou d'une procédure d'évaluation de la conformité s'il juge qu'ils constituent un obstacle technique au commerce (OEA, 1998, Encadré 5). Au contraire, certains ACR (comme les accords conclus entre le Chili et la Corée, Singapour et le Panama et le Mexique et Singapour) excluent expressément les OTC des procédures régionales de règlements des différends.

\section{Encadré 5. Règlement des différends dans la Communauté andine}

Si un pays membre estime que les normes, règlements techniques ou procédures d'évaluation de la conformité nationaux d'un autre membre constituent des obstacles techniques au commerce, celui-ci peut organiser des consultations avec le pays membre ayant adopté la mesure en question, solliciter l'intervention technique du Comité infrarégional chargé de la normalisation, de l'accréditation, des essais, de la certification, des règlements techniques et de la métrologie ou contacter le conseil pour statuer sur la conformité de la mesure avec les dispositions de l'Accord.

Si le conseil conclut qu'un obstacle existe, il peut ordonner la révocation de la mesure. La tenue de consultations, l'intervention technique du comité infrarégional ou les décisions rendues par le conseil ne peuvent dépasser un délai de trente jours calendaires à compter de la date de réception de la demande correspondante. Lorsqu'il examine l'affaire, le conseil peut solliciter l'avis technique des membres du comité infrarégional ou du comité ad hoc correspondant (Décision 376).

De 1997 à 2004, trois affaires juridiques concernant des OTC ont été engagées par des membres de la Communauté andine.

Source : OEA, 1998 ; OCDE, 2005a, http://www.comunidadandina.org/ingles/règlements.htm et Integrated Database on Trade Disputes for Latin America and the Caribbean (base de données intégrée sur les différends commerciaux concernant l'Amérique latine et les Caraïbes)

\subsubsection{Renforcement de la coopération}

\section{Assistance technique}

41. Près de $40 \%$ de l'ensemble des ACR examinés contiennent des dispositions d'assistance technique ${ }^{24}$. Généralement, les pays les plus développés comme les États-Unis (cf. Encadré 6) et les pays de l'UE (cf. étude de cas sur le Chili) s'engagent à fournir une assistance aux parties plus pauvres en proposant des formations à leurs agents publics et aux négociants et en renforçant les institutions et l'infrastructure physique nécessaires aux essais et à la certification des produits et à l'accréditation des organismes d'évaluation de la conformitée ${ }^{25}$.

24. Il ressort des études de cas que $47 \%$ des ACR examinés en moyenne contiennent des dispositions d'assistance technique. Selon Piermartini et Budetta (2006) près de $38 \%$ de tous les ACR examinés contiennent de telles dispositions.

25. De 2001 à 2005, l'aide publique au développement qui a été consacrée à la suppression des OTC (à l'échelon multilatéral, régional et bilatéral) s'est élevée à 42.2 millions USD par an. L’UE, le Japon et les États-Unis ont été les plus grands donateurs durant cette période, apportant respectivement un financement annuel moyen de 29 millions USD, 9.6 millions USD et 3.4 millions USD. Source : http://tcbdb.wto.org 


\section{Encadré 6. Assistance en matière d'OTC en vertu de l'Accord de libre-échange de l'Amérique centrale}

L'accord institue un Comité conjoint sur les affaires relatives aux OTC, qui est notamment chargé de mettre au point et de proposer des mécanismes d'assistance technique, en coopération avec le Comité sur le renforcement des capacités dans le domaine du commerce, également créé en vertu de cet accord.

Le Comité sur le renforcement des capacités dans le domaine du commerce a pour objet :

- de définir les projets prioritaires de renforcement des capacités dans le domaine du commerce à l'échelon national ou régional.

- d'inviter d'autres organismes donateurs, des entités du secteur privé et des organisations non gouvernementales à contribuer à l'élaboration et à la mise en œuvre de tels projets.

- de coopérer avec les autres comités ou groupes de travail mis en place en vertu de l'accord pour soutenir l'élaboration et la mise en œuvre de tels projets, conformément aux priorités définies dans le cadre de chaque stratégie nationale de renforcement des capacités dans le domaine du commerce.

- de suivre et d'évaluer les progrès accomplis dans la mise en œuvre de tels projets.

L'United States Agency for International Development (USAID) a dispensé des formations sur les questions relatives aux OTC aux agents publics de la République dominicaine, du Costa Rica, du Guatemala, du Salvador, du Honduras et du Nicaragua avant même l'entrée en vigueur de l'Accord en mars 2006. De 2001 à 2005, ces pays ont bénéficié (individuellement ou collectivement) d'une assistance technique en matière d'OTC, financée par les États-Unis, d'une valeur totale près de 1.3 millions USD.

Source : http://www.ustr.gov. http://tcbdb.OMC.org

\section{Métrologie}

42. En revanche, un nombre plus restreint d'ACR encourage un renforcement de la coopération dans le domaine de la métrologie, en vue de favoriser l'accréditation mutuelle des organismes de normalisation et des laboratoires ${ }^{26}$. Dans certains cas (Communauté andine, ALADI et COMESA, par exemple) ces dispositions appellent à utiliser le Système International d'Unités comme base des mesures métrologiques. D'autres accords invitent leurs membres à assurer la plus grande compatibilité possible de leurs référentiels métrologiques avec les référentiels métrologiques internationaux en vigueur. L'accord de libre-échange entre le Chili et le Costa-Rica impose ainsi aux parties «d'assurer, dans la mesure du raisonnable, la traçabilité de [leur] normes métrologiques conformément aux recommandations du Bureau international des poids et mesures (BIPM) et de l'Organisation internationale de métrologie légale (OIML)» (Article 9.09). Enfin, plusieurs dispositions (comme celles de la CARICOM) contiennent également des recommandations concernant la coopération et la coordination en matière d'infrastructure métrologique.

26. Il ressort des études de cas que $14 \%$ seulement des accords comportent des dispositions de renforcement de la coopération dans le domaine de la métrologie. Selon Piermartini et Budetta (2006), $29 \%$ des ACR examinés comportant des dispositions relatives aux OTC contiennent une clause de ce type. De même, Kotschwar (2001) observe que certains accords des ACR examinés conclus en Amérique du Nord et en Amérique latine après l'ALENA comportent des dispositions concernant la métrologie. 


\subsubsection{Conclusion}

43. La reconnaissance mutuelle des résultats des évaluations de la conformité et la transparence des mesures à caractère normatif, suivie de l'harmonisation des règlements techniques et des normes et l'acceptation des règlements des autres parties comme équivalents, est l'approche la plus couramment adoptée dans les ACR vis-à-vis des OTC (cf. Graphique 2). Ces options d'action publique sont compatibles les unes avec les autres, de sorte qu'un seul et même accord peut comporter des dispositions concernant plusieurs d'entre elles. Comme on l'a vu, aucun des accords examinés ne contient de dispositions de reconnaissance mutuelle des règlements techniques et des normes, comme celles qui sont en vigueur au sein du marché unique de l'UE. De plus, la majorité des ACR examinés prévoit la mise en place de comités chargés des affaires relatives aux OTC pour suivre la mise en œuvre des dispositions de l'accord et servir d'enceintes au sein desquelles les parties peuvent procéder à des consultations sur ces questions.

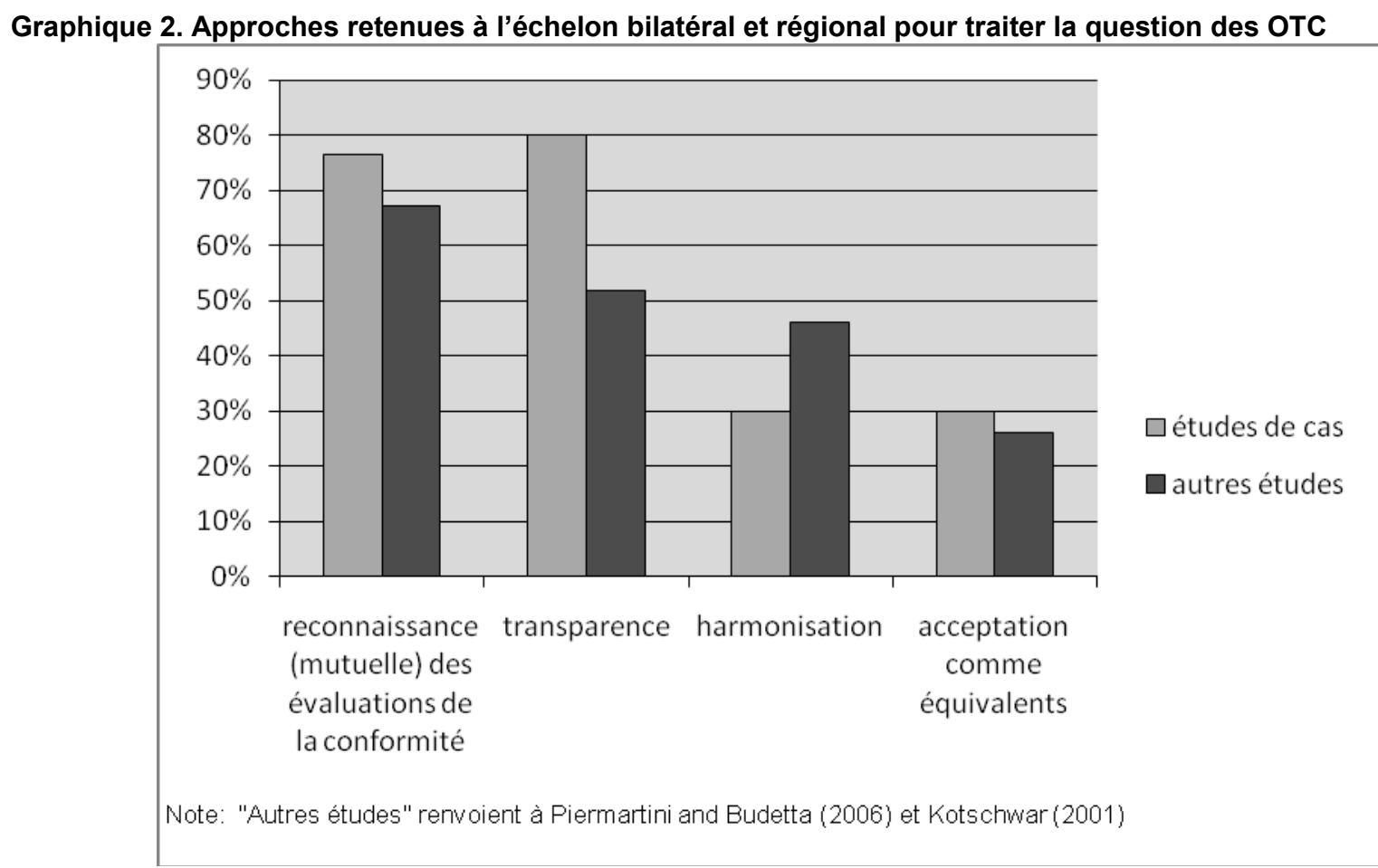

44. L'analyse montre que si une majorité des accords examinés ne comporte, dans leur globalité, pas de dispositions plus contraignantes que l'Accord sur les OTC de l'OMC, plusieurs d'entre eux affichent certaines caractéristiques allant au-delà des règles de l'OMC. Ces dispositions de plus grande portée se rapportent principalement aux obligations concernant l'acceptation des règlements techniques d'autres parties comme équivalents (dans près de $40 \%$ de tous les ACR dotés de telles clauses) et la reconnaissance (mutuelle) des résultats des évaluations de la conformité et des organismes qui en sont chargés (dans près de $25 \%$ des ACR dotés de telles clauses) imposant aux parties de donner des explications, à la demande des autres parties, en cas de non-équivalence ou de non-reconnaissance ${ }^{27}$. En outre, un petit nombre de parties (principalement des pays développés) ont conclu, parallèlement aux ACR, des accords de reconnaissance mutuelle (ARM) pour certains secteurs particuliers comme les équipements de télécommunications, électriques et électroniques et médicaux, qui vont au-delà de l'Accord sur les OTC de l'OMC. Enfin, lorsqu'un ACR établit une union douanière ou est censé constituer la première étape de la constitution d'un marché commun, des comités régionaux sur les OTC ou des organismes de

27. Cf. notes 16 et 17 . 
normalisation associés s'efforcent souvent de renforcer la coopération entre les parties, ce que ne prescrit pas l'OMC.

45. Il est malheureusement difficile d'évaluer les progrès généraux accomplis pour mettre en œuvre ces dispositions (et de les comparer à l'application de l'Accord sur les OTC de l'OMC), car il n'y a guère de documentation et d'études disponibles sur le sujet, notamment en ce qui concerne les accords de libreéchange bilatérau ${ }^{28}$. Si ces accords prévoient souvent la création de comités conjoints qui se réunissent généralement une fois par an pour suivre la mise en œuvre de ces dispositions, ces comités sont relativement peu nombreux à faire paraître des rapports d'étape concernant la mise en conformité des parties. De tels rapports sont toutefois disponibles pour la plupart des groupements et unions douanières régionaux examinés. Certains éléments factuels anecdotiques donnent à penser que les progrès les plus notables sur la voie de la suppression effective des OTC paraissent avoir été réalisés par les pays de l'UE, les membres de l'Espace économique européen, de l'ASEAN et de l'APEC (cf. chapitre IV).

\subsection{Facteurs influant sur l'approche adoptée pour réduire les OTC}

46. Baldwin (2000) souligne que le degré de libéralisation et d'harmonisation des mesures relatives aux OTC peut dépendre du niveau de développement des pays ayant conclu l'ACR. Il note que «cette libéralisation donnera presqu'assurément lieu à des accords préférentiels entre nations riches, créant, par essence, un système à deux vitesses d'accès au marché, les nations en développement se trouvant reléguées en deuxième position ». En fait, l'approche utilisée pour réduire les OTC et le degré de "libéralisation » des OTC contenus dans les ACR, sembles être influencés par trois facteurs essentiels (Piermartini et Budetta, 2006 et Kotschwar, 2001) :

- Le niveau de développement des parties impliquées

- Le degré d'intégration que l'ACR vise à réaliser.

- L'implication de l'UE ou des États-Unis dans l'ACR.

\subsubsection{Niveau de développement des parties impliquées}

47. L'harmonisation des règlements techniques et des normes, et surtout la reconnaissance mutuelle des résultats des évaluations de la conformité sont davantage susceptibles de se concrétiser entre pays similaires, ayant un niveau de développement comparable. La reconnaissance mutuelle des évaluations de la conformité est notamment plus probable entre pays dotés de capacités technologiques similaires, confiants dans leurs procédures, institutions et infrastructure réglementaires mutuelles ${ }^{29}$. L'analyse économétrique menée par Piermartini et Budetta (2006) montre ainsi que la présence de dispositions favorisant l'harmonisation des règlements techniques est positivement corrélée à la similarité du niveau de développement (RNB moyen par habitant par exemple), au degré d'intégration (comme la part des échanges commerciaux entre les parties par exemple) et au fait que l'ACR s'apparente aux accords régionaux auxquels l'UE est associée. La présence de dispositions sur la reconnaissance mutuelle des évaluations de la conformité n'est, quant à elle, positivement corrélée qu'avec la similarité du niveau de développement des parties. Singapour constitue un bon exemple à cet égard : à ce jour, tous ses accords bilatéraux ont été conclus avec des pays ayant le même niveau de développement (pays à revenu élevé,

28. Les informations sur les progrès réalisés pour mettre en œuvre l'Accord de l'OMC sur les OTC sont disponibles dans le dernier rapport du Comité de l'OMC sur les OTC (G/TBT/21/Rev.1, du 4 avril 2007). Hoekman et Kostecki (2001) note que le Comité a régulièrement organisé des réunions et qu'il n'y a eu qu'un petit nombre de différends en vertu de l'Accord sur les OTC de l'OMC.

29. $67 \%$ des ACR examinés ont été conclus entre pays ayant un niveau de développement similaire, soit $47 \%$ entre pays en développement et $20 \%$ entre pays développés. Cf. Annexe 1. 
excepté le Panama, qui est un pays à revenu moyen supérieur) et comportent des dispositions sur la reconnaissance mutuelle des résultats des évaluations de la conformité. Par ailleurs, Singapour a signé des ARM complémentaires avec la plupart de ses partenaires parties aux ACR.

48. Pourtant, ces efforts de libéralisation sont davantage susceptibles d'être effectivement mis en œuvre entre pays développés (à revenu élevé et moyen supérieur), car les pays pauvres ne disposent généralement pas des institutions et capacités nationales nécessaires pour appliquer les dispositions relatives à l'harmonisation et à la reconnaissance mutuelle. Comme on l'a vu dans la partie précédente, les mécanismes de suivi et les organismes de normalisation de ces pays n'ont souvent pas les ressources humaines et financières suffisantes ou ne bénéficient de l'intérêt soutenu des responsables politiques de haut rang qui serait nécessaire pour initier les améliorations institutionnelles à apporter. Dans le cas du Maroc, (examiné au chapitre suivant), de nombreux ACR n'imposent pas, dans la pratique, la mise en place d'un organisme distinct chargé des OTC pour gérer et superviser la mise en œuvre des dispositions relatives aux OTC qui y sont contenues. Au Chili en revanche, tous les ACR examinés préconisent la mise en place de mécanismes de suivi distincts se rapportant aux OTC. Ce pays a en outre créé un comité national sur les OTC qui contrôle notamment la conformité de toutes les obligations multilatérales, régionales et bilatérales qui incombent au Chili en matière d'OTC.

49. Enfin, la majorité des accords examinés impliquant des pays développés et en développement (comme l'accord conclu entre les États-Unis et le Maroc et l'accord EUROMED entre l'UE et le Maroc) comportent des dispositions sur l'assistance technique, destinées à renforcer les capacités, les institutions et l'infrastructure physique des pays pauvres en matière de normalisation, d'évaluation de la conformité, d'accréditation, de métrologie et de notification.

\subsubsection{Degré d'intégration visé par l'accord}

50. Une autre distinction, cette fois fondée sur le degré d'intégration visé par l'accord, peut être faite entre les ACR. Les ACR visant une intégration économique (et politique) plus poussée - comme les unions douanières, les accords visant la création d'un marché unique et les accords d'association économique tendent généralement à une harmonisation (graduelle) des règlements techniques, normes et procédures d'évaluation de la conformité, et dans une certaine mesure, des mesures métrologiques. Ils impliquent en outre le plus souvent la mise en place de mécanismes (comités) conjoints de mise en œuvre et de coopération qui vont au-delà des disciplines de l'OMC, tels l'instauration d'organismes et réseaux conjoints de normalisation et/ou d'accréditation (comme c'est le cas du MERCOSUR, cf. Encadré 7, de l'ASEAN, de la CARICOM, de la Communauté andine et des accords de coopération économique conclus par Singapour). Cela peut s'expliquer par le fait que ces accords visent généralement une meilleure harmonisation et intégration des politiques commerciales et des régimes réglementaires des parties (et poursuivent de ce fait des objectifs d'une plus grande portée que l'Accord sur les OTC de l'OMC). En revanche, les «simples » accords de libre-échange qui exigent une moindre coordination de l'action publique entre les parties (comme par exemple les accords entre pays d'Amérique latine examinés dans Kotschwar, 2001), n'encouragent souvent que la «compatibilité » ou l'équivalence des règlements techniques et des normes, et n'impliquent généralement pas une institutionnalisation aussi importante en ce qui concerne les OTC. 


\section{Encadré 7. Politique de normalisation du MERCOSUR}

L'accord du MERCOSUR vise expressément à identifier les règlements techniques "préjudiciables » et à éliminer les obstacles qu'ils créent par voie de d'harmonisation régional, de rendre compatibles les structures d'évaluation de la conformité (par voie de reconnaissance mutuelle) et de mettre au point une méthodologie commune pour élaborer un régime de normes commun pour le MERCOSUR dont le respect sera volontaire.

L'accord dispose que « dans le processus d'élaboration et de révision des règlements techniques [à caractère obligatoire], le MERCOSUR doit utiliser comme base les principes généraux et les orientations établis par l'Accord sur les obstacles techniques au commerce de l'Organisation mondiale du commerce, notamment en matière de transparence, d'information et de notification » (GMC Resolution 152/96). Le MERCOSUR a mis en place un groupe de travail ad hoc consacré à l'harmonisation des règlements techniques et à la reconnaissance mutuelle des procédures d'évaluation de la conformité.

En ce qui concerne les normes dont le respect est volontaire, l'accord établit un Comité MERCOSUR de normalisation, doté d'un directoire composé de représentants des organismes de normalisation d'Argentine, du Paraguay, du Brésil, d'Uruguay et du Chili (qui est membre associé) et instaure des Comités de normalisation sectoriels pour mener à bien ses travaux dans des domaines d'intérêt particulier. À l'heure actuelle, 16 comités de ce type travaillent sous la houlette du Comité MERCOSUR de normalisation dans le domaines suivants : énergie électrique, acier, électronique et télécommunications, jouets, ciment et béton, machines et équipements, automobile, pneumatiques, anneaux et valves, plastique, technologie de l'information, dentisterie, médecine et soins hospitaliers, papier et cellulose, soudure, et ameublement.

Ce Comité a pour objectifs principaux :

- de mettre au point des normes régionales dont le respect est volontaire.

- de promouvoir la coopération entre les membres en vue de faciliter l'harmonisation de leurs normes.

- d'harmoniser les positions politiques et techniques des membres concernant la normalisation internationale et les activités connexes.

- de promouvoir la formation dans le domaine de la normalisation et du contrôle de la qualité.

- d'encourager la mise au point de systèmes de certification et de reconnaissance mutuelle.

Source : Kotschwar, 2001, OEA, 1998

\subsubsection{Comparaison de l'approche des États-Unis et de l'Union européenne}

51. Plusieurs ACR conclus entre les États-Unis et d'autres parties visent à instaurer un processus renforcé de coopération et coordination concernant les règlements techniques, les normes et l'évaluation de la conformité, en encourageant la transparence, la responsabilisation et la coopération réglementaire entre les parties. Au nombre des options d'action publique permettant de réduire les OTC entre les parties figurent l'acceptation comme équivalents des règlements techniques de l'autre partie, l'alignement sur les normes internationales ${ }^{30}$ et la reconnaissance des résultats des évaluations de la conformité, par l'intermédiaire de mécanismes très variés (notamment la confiance de la partie importatrice dans la fiabilité des déclarations de conformité du fournisseur, les accords à caractère volontaire conclus entre les organismes d'évaluation de la conformité et l'acceptation des résultats des procédures d'évaluation de la

30. Dans de nombreux accords récents de libre-échange, les États-Unis et leurs partenaires ont convenu d'appliquer six principes (transparence, ouverture, impartialité et consensus, pertinence et efficacité, cohérence et prise en compte des intérêts du pays en développement) pour déterminer s'il existe une norme, un guide ou une recommandation internationale au sens de l'Accord sur les OTC, appliquant de ce fait la «Décision du Comité sur les principes devant régir l'élaboration de normes, guides et recommandations internationaux en rapport avec les Articles 2 et 5 et l'Annexe 3 de l'Accord » de 2002 (OMC, 2002) 
conformité effectuées sur le territoire d'une autre partie) ${ }^{31}$. Il est intéressant de noter que de nombreux ACR conclus par les États-Unis invitent les parties à expliquer pour quelles raisons elles n'acceptent pas comme équivalents les règlements techniques de l'autre partie ou les résultats de la procédure d'évaluation de la conformité menée sur le territoire d'une autre partie, lorsque celle-ci le lui demande.

52. En revanche, l'harmonisation des règlements techniques et des normes est l'option privilégiée dans la plupart des ACR examinés auxquels l'UE est partie. L'harmonisation avec les règlements et normes régionaux de l'UE est privilégiée dans les ACR conclus avec des pays candidats à l'adhésion et des pays « voisins » (comme les pays de l'EUROMED et de l'Europe du Sud-Est), avec lesquels l'UE aspire à une meilleure intégration politique et économique ${ }^{32}$. L'Accord de partenariat euro-méditerranéen (EUROMED) établissant une association entre les Communautés européennes et le Maroc appelle ainsi les parties à mettre «en œuvre les moyens propres à promouvoir l'utilisation par le Maroc des règles techniques de la Communauté et des normes européennes relatives à la qualité des produits industriels et agroalimentaires, ainsi que les procédures de certification" (Art. 40). Cela étant, l'UE favorise la convergence vers les normes internationales dans les accords conclus avec des pays plus lointains qui ne sont pas concernés par l'élargissement ou la politique de voisinage (comme le Mexique et le Chili). L'UE a en outre conclu des Protocoles à l'accord européen sur l'évaluation de la conformité et l'acceptation des produits industriels (communément appelés «PECA ») avec des pays candidats à l'adhésion, en vertu desquels la reconnaissance mutuelle s'effectue suivant le principe de l'« acquis communautaire $»^{33}$.

53. L'approche différente en matière de libéralisation retenue par l'UE et les États-Unis peut en partie s'expliquer par le fait que l'UE a mis au point un cadre institutionnel solide pour l'harmonisation des règlements techniques et des normes en vue, en premier lieu, afin de renforcer la coopération et l'harmonisation entre les États membres pour consolider le marché unique de l'UE (OCDE, 2005). L'ECDPM (à paraître) note que l'UE «a élaboré les mesures d'action publique les plus pointues et (les plus complexes) pour traiter les OTC où qu'ils se trouvent » (cf. Encadré 8).

\section{Encadré 8. Approche de l’UE en matière de règlements techniques, normes et procédures d'évaluation de la conformité}

L'UE a mis en œuvre une nouvelle approche en matière d'harmonisation technique et de normalisation en vertu de la Résolution du Conseil du 7 mai 1985. Cette approche a été conçue pour donner un nouvel élan à l'élaboration d'une législation européenne sur les produits. II s'agit d'une méthode en vertu de laquelle la législation sur les produits se limite à l'adoption d'« exigences essentielles » visant des objectifs d'intérêt collectif en matière de santé humaine, de sécurité ou d'environnement. Les spécifications techniques précises relèvent quant à elles généralement des normes volontaires européennes. Cette approche suppose qu'une vaste famille de produits peut être couverte par une seule et même législation. L' "Approche globale en matière de certification et essais " met en relief les orientations et procédures relatives aux évaluations de la conformité qui doivent être utilisées en vertu de la nouvelle approche.

Outre les règlements et les procédures d'évaluation de la conformité, dont le respect est obligatoire, l'UE a également élaboré des politiques européennes de normalisation. Au sein de l'UE, la normalisation est un processus volontaire impliquant différents acteurs économiques (pouvoirs publics, entreprises, consommateurs, ONG) et elle est

31. Sampson et Woolcock (2003) relèvent que dans la pratique, certaines de ces dispositions ont conduit à un rapprochement en direction des règlements et normes américains.

32. On entend par pays de l'EUROMED les 12 pays méditerranéens qui ont conclu la Déclaration de Barcelone de 1995 visant à donner une dimension nouvelle aux relations euro-méditerranéennes en vue de constituer une zone de libre-échange euro-méditerranéenne d'ici 2010. L'UE s'est également engagée dans un processus de stabilisation et d'association avec les pays de l'Europe du Sud-Est. Plus généralement, la Politique européenne de voisinage permet aux pays limitrophes de l'EU de renforcer leurs relations politiques et leur intégration économique avec l'Union.

33. Les Protocoles PECA diffèrent des ARM en ce qu'ils utilisent les règles techniques et les normes communes de l'UE. 
confiée à des organismes de normalisation indépendants :

- Le Comité européen de normalisation (CEN).

- Le Comité européen de normalisation électrotechnique (CENELEC).

- L'Institut européen des normes de télécommunications (ETSI).

Le modèle réglementaire de l'UE repose principalement sur le « principe de précaution » selon lequel si, après une évaluation scientifique, il est à craindre qu'un produit puisse constituer un danger éventuel et qu'une incertitude scientifique subsiste, des mesures provisoires de gestion des risques doivent être adoptées et une action préventive doit être mise en œuvre.

Source : Jenkinson, dans OCDE, 2005 et EC, 2001.

\subsection{Degré de convergence vers le système commercial multilatéral}

54. Les dispositions contenues dans les ACR renforcent le système commercial multilatéral ou convergent vers ce système lorsque les dispositions applicables à l'échelon régional reposent sur les approches sous-jacentes de l'OMC ou les dupliquent, s'inspirent d'autres accords internationaux en vigueur, ou contribuent à l'élaboration d'approches types en vue de leur éventuelle adoption ultérieure dans le dispositif de l'OMC. De plus, les ACR peuvent venir compléter les objectifs visés par le système commercial multilatéral en favorisant la coopération et l'assistance technique entre les partenaires régionaux. A l'inverse, une divergence avec le système commercial multilatéral peut se caractériser par une augmentation des coûts de transaction pour les entreprises, par des dissensions avec les règles de l'OMC, ou par une surcharge du système due à la prolifération des différends découlant des ACR conclus (OCDE, 2003).

55. L'analyse menée dans le présent document donne à penser que dans leur majorité, les dispositions relatives aux OTC contenues dans les ACR convergent vers le système multilatéral et viennent compléter les règles de l'OMC sur les OTC :

- Premièrement, la majorité des ACR conclus après 1995 (soit environ $70 \%$ des accords examinés) renvoie directement aux objectifs, droits et obligations prévus par l'Accord sur les OTC de l'OMC. Les ACR dont les objectifs vont plus loin que cet Accord ne le contredisent pas, mais visent plutôt à le promouvoir, en encourageant une meilleure coopération (économique et réglementaire) entre les parties.

- Deuxièmement, les ACR qui invitent les parties à harmoniser leurs règlements techniques, normes et procédures d'évaluation de la conformité, encouragent, dans leur majorité, la convergence des règlements techniques et procédures d'évaluation de la conformité nationaux vers les normes et guides internationaux, comme le prévoit l'Accord sur les OTC de l'OMC.

- Troisièmement, la majorité des ACR examinés encouragent les parties à reconnaître mutuellement les évaluations de la conformité effectuées par les autres parties. Près d'un quart d'entre eux vont au-delà des obligations de l'Accord de l'OMC en imposant aux parties, si on le leur demande, d'expliquer les raisons de la non-équivalence des procédures d'évaluation de la conformité ou de la non-reconnaissance des organismes d'évaluation de la conformité. On peut considérer que ces dispositions qui visent à favoriser encore plus les échanges et à supprimer les pratiques discriminatoires, viennent compléter l'Accord sur les OTC de l'OMC.

- Quatrièmement, la majorité des ACR examinés contiennent des dispositions sur la transparence qui sont en substance similaires à celles de l'Accord de l'OMC. Seul un petit nombre d'ACR examinés va plus loin en imposant, pour les notifications et les procédures d'évaluation de la 
conformité, un délai plus contraignant que celui recommandé par le Comité des OTC de l'OMC. On peut considérer, dans la pratique, que ces dispositions viennent compléter les disciplines multilatérales existantes.

- Enfin, la majorité des ACR examinés appellent à la création de comités régionaux sur les OTC afin de suivre la mise en œuvre et de servir d'enceintes au sein desquelles les parties peuvent se consulter et échanger des informations sur les questions relatives aux OTC. Dans certains cas, notamment quand on estime que l'ACR constitue la première étape de la création d'un marché commun ou vise à un renforcement de la coopération économique, ces comités assurent aussi une plus grande coopération réglementaire entre les parties (activités de normalisation conjointes à l'échelon régional et international ou coordination dans le domaine de la métrologie par exemple), ce que n'impose par l'Accord de l'OMC. De plus, un certain nombre d'ACR contiennent des dispositions spécifiques d'assistance technique. Les ACR préconisant une intensification de la coopération entre les parties en matière d'OTC sont de nature à favoriser et à renforcer la mise en œuvre de l'Accord sur les OTC de l'OMC.

56. Il peut y avoir divergence avec le système commercial multilatéral lorsqu'est encouragée, parallèlement aux normes internationales, une harmonisation avec des règlements, normes et procédures d'évaluation de la conformité régionaux. Si les règlements, normes et procédures régionaux visent généralement à combler des lacunes lorsqu'il n'existe pas encore de normes internationales (ou que cellesci sont considérées comme inappropriées) ${ }^{34}$, la coexistence de différents critères d'harmonisation peut compliquer les relations commerciales internationales. Les discussions qui ont eu lieu en 2005 lors de l'Atelier de l'OCDE sur les normes et l'évaluation de la conformité dans les échanges, ont par exemple, mis en évidence l'expérience du Mexique, pays membre de l'ALENA qui a conclu divers accords de libreéchange avec l'UE, le Japon et plusieurs pays d'Amérique centrale et du Sud - et les difficultés auxquels il est confronté en raison de l'augmentation du nombre d'ACR qui lui imposent des conditions différentes (OCDE, 2005). Dans le cas du Maroc, on peut penser que le processus d'alignement sur les mesures à caractère normatif de l'UE, (en vertu de l'accord l'EUROMED) pourrait se trouver compliqué s'il advenait que des personnes morales américaines étaient habilitées à prendre part à l'élaboration des normes, règlements techniques et procédures d'évaluation de la conformité du Maroc (cf. accord de libreéchange entre le Maroc et les États-Unis).

57. De plus, si les ARM sont encouragés par, et reposent sur, l'Accord sur les OTC de l'OMC, ils peuvent, dans certains cas, porter préjudice aux exportations d'un pays tiers et s'éloigner, de ce fait, de l'esprit de non-discrimination promu par le système multilatéral (Amurgo Pacheco, 2006, et Chen et Mattoo, 2004) $)^{35}$. Les obligations imposant un renforcement de la transparence et un plus grand recours aux ARM, mais surtout le fait de faciliter l'adhésion de pays tiers, pourraient toutefois contrebalancer certains de ces effets préjudiciables.

58. Cela étant, le plus grand danger que peuvent poser les ACR pour le système commercial multilatéral tient au fait que les capacités humaines et institutionnelles sont susceptibles d'être détournées

34. La Communication de la Commission européenne sur la politique commerciale extérieure de la communauté dans le domaine des normes et de l'évaluation de la conformité mentionne ainsi que le régime de l'UE offre un bon modèle de conformité avec l'Accord sur les OTC [de l'OMC]. Les Directives harmonisées respectent ses principales exigences. Les normes - qui reposent largement sur des normes internationales - sont pour la plupart volontaires et les exigences réglementaires ne sont imposées que lorsque cela est nécessaire pour des raisons liées de santé, de sécurité ou d'autres motifs légitimes » (CE, 1996).

35. Chen et Mattoo, 2004, observent ainsi que les ARM relatifs aux procédures d'évaluation de la conformité peuvent léser des pays tiers si leur champ d'application est limité par les règles d'origine. 
des efforts multilatéraux, pour être prioritairement consacrées aux mesures régionales (Fiorentino et Crawford, 2005). Le manque de ressources humaines et financières des secteurs public et privé et les capacités insuffisantes qui en découlent pour participer activement à la fois aux discussions de l'OMC et aux activités de normalisation internationale d'une part et aux négociations et activités de libéralisation régionale d'autre part est une préoccupation particulière pour les pays en développement. Comme on l'a $\mathrm{vu}$, les efforts menés à l'échelon régional pour réduire les OTC impliquent souvent la participation (obligatoire ou volontaire) à un grand nombre d'institutions. 
TAD/TC/WP(2007)12/FINAL

\section{IV. ÉTUDES DE CAS}

59. Les ACR constituent souvent l'une des composantes d'un effort politique plus vaste pour approfondir les relations économiques avec les pays voisins. Comme les accords prolifèrent, un pays devient souvent membre de plusieurs accords différents. Selon la Banque mondiale (2005), un pays africain moyen est partie à quatre accords différents et un pays latino-américain moyen à sept accords. La prolifération des accords peut entraîner des chevauchements dans la réglementation et une hausse des coûts administratifs. Parallèlement, les ACR peuvent améliorer les connaissances administratives concernant les négociations et la supervision des dispositions sur les OTC.

60. Ce chapitre analyse plus en profondeur les ACR ratifiés concernant, respectivement, le Chili, Singapour et le Maroc. Chaque étude de cas examine les dispositions sur les OTC comprises dans un échantillon d'ACR que le pays a conclus, en s'intéressant en particulier à leur cohérence entre eux et avec les disciplines de l'OMC, et à l'infrastructure institutionnelle mise en place pour traiter le large éventail des dispositions sur les OTC.

\subsection{Chili $^{36}$}

\subsubsection{Introduction}

61. Jusqu'en 1990, la politique commerciale chilienne reposait principalement sur une libéralisation unilatérale. Cela étant, depuis environ 1995, le Chili a cherché de plus en plus à s'ouvrir au commerce et à l'investissement en concluant des accords commerciaux préférentiels bilatéraux, régionaux et plurilatéraux, ainsi qu'à travers une libéralisation unilatérale et une participation au système commercial multilatéral (OMC, 2003). Il a d'abord conclu des ACR avec les pays voisins d'Amérique latine et centrale (de 1995 à la fin des années 90), puis avec les principaux blocs commerciaux (UE, États-Unis et AELE, 2003/4), et plus récemment avec les pays de la région Asie-Pacifique (Corée, Chine et Nouvelle-Zélande, Singapour, Brunei Darussalam, 2004/6). En outre, il négocie actuellement des protocoles bilatéraux avec le Guatemala, le Honduras et le Nicaragua, qui sont membres du Marché commun de l'Amérique centrale (Direcon, 2007).

62. Le nombre croissant d'ACR que le Chili a conclus a nécessité la mise en place d'institutions dédiées à la mise en œuvre et l'administration de ces accords. Parmi les missions de ces institutions, on compte l'application intégrale des ACR, le contrôle de la conformité avec les engagements mutuels, la gestion des ACR pour s'assurer du fonctionnement efficace de leur structure institutionnelle respective et la maximisation de l'utilisation des avantages et opportunités générés par les accords. En outre, la prolifération des ACR requiert également une coordination de tous les intervenants concernés : le secteur public, le pouvoir législatif, le secteur privé et la société civile (Direcon, 2007).

\subsubsection{Analyse comparative des dispositions régionales sur les OTC}

63. Le Chili attache une grande importance à la suppression des OTC, tant aux niveaux bilatéraux, que régionaux et multilatéraux. Il participe à trois groupements régionaux qui œuvrent à l'élimination des OTC - l'APEC (en tant que membre), le MERCOSUR (en tant que pays associé) et l'Association latinoaméricaine d'intégration (en tant que membre). En outre, depuis avril 2007, le Chili a conclu 17 accords de libre-échange et de complémentarité économique qui contiennent des dispositions sur les OTC et qui sont entrés en vigueur (tableau 3). Il avait alors aussi conclu deux ARM concernant les pratiques œnologiques

36. Cette étude de cas a été relue par Ana Maria Vallina, responsable du Département du commerce extérieur du ministère chilien de l'Économie. Mme Vallina a fourni des informations précieuses concernant les systèmes juridiques et institutionnels du Chili. 
(voir plus loin). Cette section analyse le texte de 10 accords comportant des dispositions relatives aux OTC (voir tableau 3 , lignes surlignées) ${ }^{37}$.

Tableau 3. Vue d'ensemble des ACR du Chili, au 2 avril 2007

\begin{tabular}{|c|c|c|c|c|c|}
\hline $\mathrm{N}^{\circ}$ & Accord & Entrée en vigueur & Type d'accord & $\begin{array}{l}\text { Notification } \\
\text { à l'OMC }\end{array}$ & $\begin{array}{c}\text { Dispositions sur } \\
\text { les OTC }\end{array}$ \\
\hline 1 & Chili-Canada & 5 juillet 97 & Accord de libre-échange & oui & $\begin{array}{c}\text { Secteur des } \\
\text { télécommunications }\end{array}$ \\
\hline 2 & Chili-Mexique & 1er août 99 & Accord de libre-échange & oui & oui \\
\hline 3 & Chili-Costa Rica** $^{* *}$ & 14 février 02 & Accord de libre-échange & oui & oui \\
\hline 4 & Chili- Salvador** & 3 juin 02 & Accord de libre-échange & oui & oui \\
\hline 5 & Chili-UE $^{*}$ & 1er février 03 & Accord de libre-échange & oui & oui \\
\hline 6 & Chili-États-Unis & 1er janvier 04 & Accord de libre-échange & oui & oui \\
\hline 7 & Chili-République de Corée & 1er avril 04 & Accord de libre-échange & oui & oui \\
\hline 8 & Chili-AELE & 1er décembre 04 & Accord de libre-échange & oui & oui \\
\hline 9 & Chili-Chine & 1er octobre 06 & Accord de libre-échange & non & oui \\
\hline 10 & $\begin{array}{l}\text { Partenariat économique } \\
\text { stratégique transpacifique* } \\
\text { (Chili, Nouvelle-Zélande, } \\
\text { Singapour, Brunei Darussalam) }\end{array}$ & 8 novembre 06 & Accord de libre-échange & non & oui \\
\hline 11 & $\begin{array}{l}\text { Association latino-américaine } \\
\text { d'intégration }\end{array}$ & 18 mars 81 & Accord préférentiel & oui & oui \\
\hline 12 & Amérique centrale & (signé le 18 oct. 99) & Accord de libre-échange & non & oui \\
\hline 13 & Chili-Argentine $^{\star \star *}$ & 2 août 91 & $\begin{array}{c}\text { Accord de complémentarité } \\
\text { économique }\end{array}$ & non & oui \\
\hline 14 & Chili-Venezuela $^{\star * *}$ & 1er juillet 93 & $\begin{array}{c}\text { Accord de complémentarité } \\
\text { économique }\end{array}$ & non & oui \\
\hline 15 & Chili-Bolivie & 07 juillet 93 & $\begin{array}{l}\text { Accord de complémentarité } \\
\text { économique }\end{array}$ & non & oui \\
\hline 16 & Chili-Colombie $^{\star \star *}$ & 1er janvier 94 & $\begin{array}{c}\text { Accord de complémentarité } \\
\text { économique }\end{array}$ & non & oui \\
\hline 17 & Chili-Équateur $^{* \star *}$ & 1er janvier 95 & $\begin{array}{c}\text { Accord de complémentarité } \\
\text { économique }\end{array}$ & non & oui \\
\hline 18 & MERCOSUR & 1er oct. 96 & $\begin{array}{l}\text { Accord de complémentarité } \\
\text { économique }\end{array}$ & non & oui \\
\hline 19 & Chili-Pérou $^{\star \star \star}$ & 1er juillet 98 & $\begin{array}{c}\text { Accord de complémentarité } \\
\text { économique }\end{array}$ & non & oui \\
\hline 20 & Chili-Inde & (signé le 8 mars 06) & Accord de portée partielle & non & oui \\
\hline 21 & Chili-Panama & (signé le 27 juin 06) & Accord de libre-échange & non & s.o. \\
\hline 22 & Chili-Japon & (signé le 27 mars 07) & Accord de libre-échange & non & oui \\
\hline 23 & Chili-Cuba & $\begin{array}{l}\text { Procédure parlementaire } \\
\text { en cours }\end{array}$ & Accord de portée partielle & non & s.o. \\
\hline
\end{tabular}

Source : http://www.direcon.cl/cuadro_resumen_en.html

${ }^{*}$ Accord d'association économique

** Protocole bilatéral à l'Accord de libre-échange de l'Amérique centrale

*** Accord de libre-échange conclu pour compléter l'Association latino-américaine d'intégration.

37. L'approche de l'APEC relative à la suppression des OTC est analysée dans la prochaine section (étude de cas sur Singapour). 
64. La totalité des dix ACR examinés fait directement référence à l'Accord sur les OTC de l'OMC et réaffirme les droits et obligations mutuels des parties aux termes de l'Accord sur les obstacles techniques au commerce. Les ACR réitèrent tous la nécessité d'éviter les obstacles non nécessaires au commerce et la discrimination entre les parties. Certains ACR cherchent à faciliter le commerce au-delà des dispositions de l'OMC à travers une amélioration de la coopération en matière de réglementation (par exemple, le Partenariat économique stratégique transpacifique et l'accord d'association UE-Chili). De même, l'accord entre le Chili et les États-Unis appelle les parties à identifier d'autres initiatives de collaboration adaptées à des aspects ou des secteurs particuliers.

\section{Harmonisation, compatibilité et acceptation des règlements techniques comme équivalents}

65. Les accords conclus le plus récemment (avec l'UE, la Corée, la Chine et le Partenariat économique stratégique transpacifique) imposent spécifiquement aux parties d'harmoniser leurs règlements techniques et les procédures connexes d'évaluation de la conformité sur la base des normes internationales lorsqu'elles existent ou que leur finalisation est imminente, sauf quand ces normes constituent «un moyen inefficace ou inapproprié de réaliser les objectifs légitimes poursuivis». Parallèlement à l'harmonisation, ces accords encouragent également l'équivalence et la compatibilité des règlements techniques. L'accord d'Association économique avec l'UE, par exemple, appelle les parties à s'efforcer d'« accroître la compatibilité et/ou l'équivalence de leurs réglementations techniques, normes et procédures d'évaluation de la conformité respectives » (article 87), tandis que le Partenariat économique stratégique transpacifique appelle les parties à envisager «d'accepter comme équivalents les règlements techniques d'une autre partie » (article 8.7). Il est intéressant de constater que plus de la moitié des accords examinés exigent des parties qu'elles expliquent les raisons pour lesquelles elles refusent l'équivalence des règlements techniques d'autres parties, et qu'ils vont par conséquent au-delà de l'Accord sur les OTC de l'OMC.

66. En revanche, les ACR plus anciens, conclus essentiellement avec les voisins latino-américains (Mexique, Costa Rica et Salvador), exigent des parties qu'elles parviennent à une "compatibilité »des mesures liées aux normes, sans préciser si l'approche de libéralisation privilégiée reposerait sur l'harmonisation ou l'équivalence (les deux sont cohérentes avec l'Accord sur les OTC de l'OMC). Les ACR avec le Mexique, par exemple, stipulent que «les parties devront, dans toute la mesure du possible, rendre compatibles leurs mesures respectives sans réduire la sécurité ou la protection de la vie humaine, animale ou végétale ou de la santé, de l'environnement ou des consommateurs [...] et en prenant en compte les activités internationales de normalisation » (article 8). ${ }^{38}$

\section{Reconnaissance mutuelle des évaluations de la conformité}

67. Tous les ACR examinés à l'exception de l'Accord Chili-AELE comportent des dispositions sur la reconnaissance mutuelle des résultats des évaluations de la conformité. Les accords de libre-échange conclus par le Chili encouragent généralement les parties à négocier des accords de reconnaissance mutuelle, à se fier à la déclaration de conformité des fournisseurs et à accréditer les organismes d'évaluation de la conformité situés sur le territoire d'autres parties. Enfin, plus de la moitié des ACR

38. Il n’y a pas de définition officielle de la «compatibilité ». Dans plusieurs des ACR examinés, elle est définie ainsi : «[...] rapprocher différentes mesures à caractère normatif de même portée qui ont été approuvées par différentes instances de normalisation de sorte qu'elles soient identiques, ou équivalentes, ou bien où qu'elles permettent d'utiliser des biens ou des services de façon interchangeable ou de remplir le même objectif (par exemple Chili-Costa-Rica). 
examinés vont au-delà des règles de l'OMC dans le sens où les parties doivent expliquer les raisons de la non-reconnaissance (accords avec le Costa Rica, le Salvador, la Corée, les États-Unis, la Chine et le Partenariat économique stratégique transpacifique).

68. En outre, le Chili est, depuis 2003, membre de l'Accord d'acceptation mutuelle des pratiques oenologiques (parmi les autres membres figurent l'Argentine, l'Australie, le Canada, les États-Unis et la Nouvelle-Zélande) dans le cadre duquel les parties reconnaissent mutuellement leur législation, leur réglementation et leurs exigences relatives aux pratiques œnologiques et un mécanisme de règlement des différends est prévu par l'intermédiaire d'un conseil et d'un comité d'experts. L'Accord appelle les parties à accepter mutuellement les mécanismes en place destinés à aplanir les différends à l'échelon bilatéral. Le Chili a en outre conclu un ARM avec l'UE (2003) concernant les pratiques œnologiques et l'étiquetage des vins, qui autorise les parties à importer du vin produit et étiqueté chez l'autre partie conformément aux pratiques viticoles réciproquement acceptées.

4.

Transparence

69. La majorité des ACR du Chili examinés (8 sur 10) comportent des dispositions sur la transparence imposant aux parties de notifier aux autres parties les règlements techniques et les procédures d'évaluation de la conformité récemment modifiés ou adoptés, quand ceux-ci diffèrent des normes internationales ou sont susceptibles d'avoir un effet sur les échanges, même si aucun accord n'exige la création de points d'information distincts. Ces dispositions convergent vers les disciplines de l'OMC, en précisant que chaque partie notifiera les règlements techniques et les procédures d'évaluation de la conformité aux autres parties 60 jours au moins avant leur adoption, "pour permettre aux parties intéressées de soumettre leurs observations et leurs consultations pendant cette période et pour permettre à la partie notifiante d'en tenir compte" (autrement dit, les accords avec le Mexique, le Costa Rica, le Salvador, les États-Unis, la Corée, la Chine et le Partenariat économique transpacifique). En outre, trois accords (respectivement avec la Corée, le Mexique et les États-Unis) autorisent les personnes d'une partie (secteur public ou privé) à participer à l'élaboration de normes, règlements techniques et procédures d'évaluation de la conformité de l'autre partie.

\section{5. $\quad$ Mise en ouvre des accords et règlement des différends}

70. Tous les ACR examinés du Chili prévoit la création d'un comité conjoint pour contrôler la mise en œuvre de l'accord et permettre aux parties de se consulter sur les questions concernant les OTC. Certains ACR créent des institutions pour gérer les efforts en vue d'une coopération plus avancée dans le domaine de l'harmonisation, l'équivalence et l'accréditation, par exemple Le Comité spécifique Chili-UE sur les règlements techniques, normes et évaluations de la conformité (encadré 9) ou le Comité établi par l'intermédiaire du Partenariat économique stratégique transpacifique. Deux accords en particulier instaurent des instances sectorielles pour traiter des normes de télécommunication (accords avec le Canada et le Mexique). De plus, la majorité des ACR du Chili examinés comportent des dispositions sur la résolution des différends entre les membres par voie de consultations. 
TAD/TC/WP(2007)12/FINAL

\section{Encadré 9. Comité spécial des règlementations techniques, des normes et des évaluations de la conformité} instauré par l'Accord Chili-UE

Le Comité est co-présidé par le Chili et l'UE. II a établi un programme de travail en vue réaliser les objectifs des ACR concernant la coopération renforcée. II a les compétences et exerce les fonctions suivantes :

- $\quad$ surveiller la mise en œuvre et la gestion du chapitre sur les OTC,

- offrir une enceinte de débat et d'échange d'informations sur toute question en relation avec le chapitre sur les OTC, en particulier avec les systèmes mis en place pour les règlementations techniques, les normes et les procédures d'évaluation de la conformité, ainsi que sur l'évolution de la situation dans les organisations internationales compétentes dans ces domaines,

- $\quad$ servir de lieu de consultation entre les parties et de résolution rapide des problèmes qui font ou sont susceptibles de faire inutilement obstacle au commerce, dans les limites du champ d'application et de l'objet de ce chapitre,

- $\quad$ encourager, promouvoir et faciliter par ailleurs la coopération entre les organisations des parties, publiques et/ou privées, chargées de la métrologie, de la normalisation, des essais, de la certification, des inspections et l'accréditation, ainsi que

- $\quad$ explorer tous les moyens permettant d'améliorer l'accès aux marchés respectifs des parties et l'amélioration du fonctionnement de ce chapitre.

Le Comité a également défini et lancé un important programme d'assistance technique financé par l'UE (voir ciaprès).

Source : http://www.direcon.cl

\section{Renforcement de la coopération}

71. La plupart des accords examinés (sauf ceux avec l'AELE, le Canada et le Partenariat économique stratégique transpacifique) comportent des dispositions concernant l'assistance technique. Quatre accords vont au-delà des dispositions de l'OMC, en encourageant une collaboration dans le domaine de la métrologie, sur la base des recommandations et des guides de l'Organisation internationale de métrologie légale (accords avec l’UE, le Mexique, le Costa Rica et le Salvador).

\section{Conclusion}

72. Tous les accords examinés réaffirment les droits et les obligations des parties aux termes de l'Accord sur les OTC de l'OMC. Certaines dispositions sur les OTC dans les ACR du Chili vont au-delà des règles de l'OMC, en particulier concernant l'acceptation de l'équivalence des règlements techniques et la reconnaissance mutuelle des procédures d'évaluation de la conformité, en vertu desquelles, dans la plupart des cas, les parties doivent expliquer, lorsqu'on leur en fait la demande, les raisons d'une nonéquivalence ou d'une non-reconnaissance. On peut considérer que ces dispositions convergent vers le système commercial multilatéral car elles cherchent à renforcer encore la transparence et la nondiscrimination.

73. De plus, un certain nombre d'ACR du Chili visent une coopération plus ambitieuse dans le domaine de la réglementation et créent des comités conjoints chargés non seulement du contrôle, de la gestion du chapitre sur les OTC et des consultations qui s'y rapportent, mais aussi d'autres initiatives de collaboration dans le domaine de l'harmonisation, de l'équivalence, de l'accréditation et de l'assistance technique (par exemple, les accords d'association avec l'UE et le Partenariat économique stratégique transpacifique) ou dans le domaine de la métrologie (accords avec le Mexique, le Costa Rica et le Salvador). On peut considérer que ces dispositions, tout en allant au-delà des règles multilatérales, complètent l'Accord de l'OMC. 
74. Enfin, il semble exister une certaine cohérence entre les chapitres sur les OTC contenus dans les différents ACR qu'a conclus le Chili. Premièrement, presque tous les accords examinés appellent à une reconnaissance mutuelle des procédures d'évaluation de la conformité. Cela peut s'expliquer par le fait qu'ils ont généralement été conclus avec des pays ayant un niveau de développement semblable ou supérieur à celui du Chili, qui sont donc susceptibles d'avoir des procédures et une infrastructure d'évaluation de la conformité auxquelles le Chili peut se fier et sur lesquelles il peut s'appuyer. Deuxièmement, la majorité des ACR comportent aussi des dispositions identiques concernant la transparence des mesures à caractère normatif. Enfin, la plupart des ACR encouragent soit la " compatibilité » de ces mesures soit à conjuguer harmonisation avec les normes internationales et équivalence des règlements techniques.

\subsubsection{Dispositif institutionnel de gestion des OTC}

75. Le Chili a mis en place une solide structure institutionnelle pour traiter des OTC aux niveaux bilatéral, régional et multilatéral. En mai 1995, l'Accord sur les OTC de l'OMC a été transposé dans le droit de la République du Chili, après l'approbation d'une loi à cet effet par le Congrès national. La responsabilité de la mise en œuvre et de l'administration de l'Accord sur les OTC de l'OMC relève du ministère de l'Économie, du Développement et de la Reconstruction, et plus spécifiquement de son Département du Commerce extérieur. Le Département intervient comme Point national d'information du Chili pour les règlements techniques et les procédures d'évaluation de la conformité, instance mise en place dans le cadre des engagements du pays vis-à-vis de l'OMC (OMC, 2003).

76. La législation du Chili concernant la notification, l'élaboration, l'adoption et la mise en œuvre des règlements techniques et des procédures d'évaluation de la conformité s'inspire des règles de l'OMC (OMC, 2006). La loi 19.912 sur les questions relatives aux OTC (2003) et le Décret sur les bonnes pratiques en matière de réglementation connexe, par exemple, appellent à :

- utiliser les normes internationales comme base pour élaborer les règlements et les normes nationaux,

- éviter les obstacles non nécessaires au commerce,

- respecter les principes de traitement national et de la nation la plus favorisée,

- ménager un délai de consultation publique sur les projets de règlements techniques de 60 jours au moins et fournir des informations au ministère de l'Économie pour qu'il puisse en informer l'OMC et

- ménager un délai d'au moins 6 mois entre l'adoption et la publication de règlements techniques et leur entrée en vigueur, pour donner aux opérateurs économiques le temps de s'adapter aux nouvelles dispositions. Dans certaines circonstances, ce délai peut être écourté (OMC, 2006 et Mme Vallina, ministère de l'Économie, Chili).

77. Le Décret, qui a été élaboré par le Comité national des obstacles techniques au commerce, prévoit des règles claires et identiques applicables à tous les organismes publics concernés sur l'élaboration, l'adoption et la mise en œuvre des règlements techniques et des procédures d'évaluation de la conformité (OMC, 2003).

78. De plus, un Comité national des obstacles techniques au commerce a été mis en place en 1997. Il est placé sous la direction du ministère de l'Économie et comprend des représentants de tous les ministères et organismes publics intervenant dans la préparation, l'adoption, l'application et le contrôle de la mise en œuvre des règlements techniques (par exemple les ministères de la Santé et de l'Agriculture, et le Sous-secrétariat aux télécommunications), de même que des représentants de l'Institut national de 
normalisation, chargé du développement et de la mise en œuvre de normes volontaires, et du ministère des Affaires étrangères, chargé de l'autorité chilienne pour la promotion des exportations. Cette Commission, entre autres, coordonne la position du Chili face aux problèmes concernant les OTC dans les accords préférentiels et au sein de l'OMC. Par conséquent, elle permet d'éviter les incohérences entre les dispositions sur les OTC des différents ACR et les divergences par rapport aux règles de l'OMC (encadré $10)$.

\section{Encadré 10. Responsabilités essentielles du Comité national chilien des obstacles techniques au commerce}

Le Comité national a pour objectif de :

- coordonner et assurer la cohérence entre les efforts des différentes instances intervenant dans les questions de normalisation dans le pays. La coordination est assurée par les points de contact dans chaque organisme de réglementation représenté au sein de la Commission.

- $\quad$ prendre des mesures pour renforcer la surveillance du marché et s'assurer que les produits proposés sur le marché chilien sont conformes aux règlements obligatoires.

- $\quad$ assurer la conformité avec les obligations OTC de l'OMC et fournir des mises à jour régulières aux instances chiliennes de réglementation sur les activités de l'OMC ou concernant les OTC.

- participer aux travaux du Sous-comité sur les normes et la conformité de l'APEC et en informer les organismes de réglementation.

- coordonner la position du pays vis-à-vis des problèmes concernant les OTC dans les négociations commerciales bilatérales, régionales et plurilatérales et suivre et administrer les chapitres sur les OTC contenus dans les ACR du Chili et soutenir leur mise en œuvre.

- coordonner la position du pays concernant toute autre question d'ordre international relative aux OTC (par exemple la nouvelle réglementation européenne sur les produits chimiques $\mathrm{REACH}$ ).

- aborder la question de la normalisation comme instrument visant à soutenir le processus de modernisation technologique du pays

- identifier les besoins de renforcement des capacités et fournir une formation aux organismes de réglementation et aux entreprises concernés.

Source : OMC, 2006 et informations fournies par Mme Ana Maria Vallina

79. De plus, pour améliorer la transparence et la disponibilité publique des règlements techniques, un site Internet ${ }^{39}$ où peuvent être consultés tous les règlements techniques du Chili et de 1'UE a été créé, sous l'autorité du ministère de l'Économie et de ProChile, l'agence publique de promotion des exportations, avec la participation de tous les organismes intervenant dans la Commission nationale sur les obstacles techniques au commerce. Ce site Internet a été financé par l'UE dans le contexte du programme de coopération de l'UE engagé dans le cadre de l'accord d'association UE-Chili ${ }^{40}$.

80. Enfin, les normes sont élaborées par l'Institut national de normalisation (INNO) du Chili, qui a adopté le Code de pratique pour l'élaboration, l'adoption et l'application des normes (d'application volontaire) de l'OMC. L'Institut encourage et facilite le recours aux normes internationales au niveau

\footnotetext{
39. http://www.reglamentostecnicos.cl/

40. Informations fournies par Mme Vallina
} 
national ${ }^{41}$ et sert de point national d'information sur les normes. Les normes sont évaluées par rapport aux référentiels ISO et CEI, internationalement reconnus et voient le jour à travers un processus de consultations entre représentants du secteur public et du secteur privé (le processus de consultation publique est ouvert pendant 60 jours). L'Institut national de normalisation accrédite également des organismes qui délivrent des certificats d'évaluation de la conformité pour les produits d'exportation (OMC, 2006 et OMC, 2003). En outre, le Chili est aussi membre de la Commission panaméricaine de normalisation (COPANT), l'Organisation internationale de normalisation (ISO), le Système interaméricain de métrologie et la Coopération interaméricaine d'accréditation (OMC, 2003).

\subsection{Singapour ${ }^{42}$ \\ 4.2.1 Introduction}

81. Singapour est un pays très dépendant des échanges qui a mené une politique commerciale articulée autour de trois axes aux niveaux bilatéral, régional et multilatéral ${ }^{43}$. Il est traditionnellement un fervent défenseur du système commercial multilatéral et assure un traitement de la nation la plus favorisée (NPF) à tous les membres de l'OMC. Il est aussi membre de l'ASEAN, de l'APEC, et des Rencontres Asie-Europe (ASEM). Depuis 2000, Singapour cherche plus activement à conclure des accords bilatéraux de libre-échange, d'abord avec les pays voisins de la région Asie-Pacifique (2001/03), puis avec deux grands blocs commerciaux (l'AELE et les États-Unis, 2003/04), et tout récemment avec des pays d'autres régions (Jordanie, 2005, Panama, 2006 et Chili, à travers le Partenariat économique stratégique transpacifique, 2006). Depuis avril 2007, il a engagé des négociations en vue d'un accord de libre-échange bilatéral avec le Canada, la Chine, le Conseil de coopération du Golfe, le Pakistan et le Pérou, ainsi que des négociations multilatérales, en même temps que d'autres membres de l'ASEAN, avec l'Australie et la Nouvelle-Zélande, l'Inde, le Japon et, plus récemment, avec l'UE (International Enterprise Singapore, 2007).

\subsubsection{Analyse comparative des dispositions régionales sur les OTC}

82. Au-delà de ses obligations multilatérales, Singapour est tenu par des nombreuses obligations bilatérales et régionales en rapport avec les OTC. Il appartient à trois groupements commerciaux régionaux, l'ASEAN, l'APEC et l'ASEM, qui travaillent sur les questions concernant les OTC. Depuis avril 2007, le pays a conclu 10 accords de libre-échange bilatéraux et plurilatéraux qui sont entrés en vigueur et qui contiennent des dispositions sur les OTC (tableau 4).

41. D'après les autorités chiliennes, $70 \%$ de l'ensemble des normes nationales élaborées sont fondées sur des normes internationales (OMC, 2003).

42. Cette étude de cas a été relue par M. Sze Gin Low, directeur adjoint senior, OMC et négociations commerciales internationales, ministère du Commerce et de l'Industrie de Singapour. M. Low et ses collègues de SPRING Singapour et l'Infocomm Development Authority ont fourni de précieux éléments d'informations pour l'étude.

43. En 2004, le commerce de marchandises à Singapour était équivalent à trois fois son PIB. L'économie est extrêmement dépendante des marchés extérieurs pour sa croissance économique (OMC, 2004). 
Tableau 4. Vue d'ensemble des ACR de Singapour, au 2 avril 2007

\begin{tabular}{|c|c|c|c|c|c|}
\hline $\mathbf{N}^{\circ}$ & Accord & Entrée en vigueur & Type d'accord & $\begin{array}{l}\text { Notification } \\
\text { à l'OMC }\end{array}$ & $\begin{array}{l}\text { Dispositions } \\
\text { sur les OTC }\end{array}$ \\
\hline 1 & $\begin{array}{l}\text { Accord de libre-échange de } \\
\text { l'ASEAN (AFTA)** }\end{array}$ & 28 janvier 92 & Accord préférentiel & oui & oui \\
\hline 2 & $\begin{array}{l}\text { Singapour-Nouvelle- } \\
\text { Zélande* }\end{array}$ & $1^{\mathrm{er}}$ janvier 01 & Accord de libre-échange & oui & oui \\
\hline 3 & Singapour-Japon* & 30 novembre 02 & Accord de libre-échange & oui & oui \\
\hline 4 & Singapour-AELE & $1^{\mathrm{er}}$ janvier 03 & Accord de libre-échange & oui & oui \\
\hline 5 & Singapour-Australie* & 28 juillet 03 & Accord de libre-échange & oui & oui \\
\hline 6 & Singapour-États-Unis* & 1 janvier 04 & Accord de libre-échange & oui & oui \\
\hline 7 & Singapour-Corée* & 2 mars 06 & Accord de libre-échange & oui & oui \\
\hline 8 & Singapour-Panama & 24 juillet 06 & Accord de libre-échange & non & oui \\
\hline 9 & $\begin{array}{l}\text { Partenariat économique } \\
\text { stratégique transpacifique* }\end{array}$ & 08 novembre 06 & Accord de libre-échange & non & oui \\
\hline 10 & Singapour-Inde* & $1^{\text {er }}$ août 2005 & Accord de libre-échange & non & oui \\
\hline 11 & Singapour-Jordanie & 22 août 05 & Accord de libre-échange & oui & non \\
\hline 12 & ASEAN-Chine & $1^{\mathrm{er}}$ juillet 03 & Accord préférentiel & non & oui \\
\hline 13 & ASEAN-Corée & $\begin{array}{l}\text { (Chapitre sur le commerce } \\
\text { des marchandises signé en } \\
\text { 2005) }\end{array}$ & Accord préférentiel & non & oui \\
\hline
\end{tabular}

Source : http://www.iesingapore.gov.sg/ et portail régional de l'OMC

* Partenariat économique complet ou accords de coopération économique

83. Singapour a aussi conclu plusieurs Accords de reconnaissance mutuelle (ARM) - soit dans le cadre de ses ACR, soit sous forme d'accords «autonomes » avec des parties avec lesquelles il n'a pas encore conclu d'ACR (par exemple, le Canada et Hong-Kong). Cette section analyse le texte de 10 ACR comportant des dispositions sur les OTC (tableau 4, lignes surlignées) et une sélection d'ARM gouvernementaux (tableau 5), ainsi que l'approche (volontaire) de l'APEC en vue de la suppression des OTC.

\section{Référence à l'Accord sur les OTC de l'OMC}

84. Tous les accords examinés réaffirment les objectifs multilatéraux en matière d'OTC, ainsi que les droits et les obligations des parties aux termes de l'Accord sur les OTC de l'OMC. De plus, la moitié des accords examinés font aussi référence à des objectifs plus généraux, comme l'amélioration des conditions pour les entreprises (accord Singapour-Corée), la facilitation de l'investissement (accord SingapourAustralie) ou le renforcement de la coopération dans le domaine de la réglementation, objectif surtout intégré dans les ACR plus récents (par exemple, le Partenariat économique stratégique transpacifique et les accords avec la Corée et le Panama).

\section{Harmonisation des règlements techniques, normes et évaluations de la conformité}

85. La majorité des ACR de Singapour examinés encouragent les parties à aligner leurs règlements techniques et normes sur les normes internationales lorsqu'elles existent et sont jugés appropriés (par exemple, les accords au sein de l'ASEAN, et avec la Nouvelle-Zélande, l'Australie, la Corée, le Panama, l'Inde et le Partenariat économique stratégique transpacifique). Au sein de l'ASEAN, l'harmonisation des règlements techniques obligatoires progresse à un rythme différent dans les quatre secteurs (produits électriques et électroniques, et cosmétiques, pour lesquels des accords ont été conclus; produits pharmaceutiques, pour lesquels des Exigences techniques communes et un Dossier technique commun ont 
été remplis ${ }^{44}$; et pour les préparations alimentaires, pour lesquelles il n'existe pas encore d'accord formel). De plus, une harmonisation des normes volontaires a été réalisée pour 20 produits (OCDE, 2005 et informations fournies par M. Low).

86. Parallèlement aux normes internationales, l'ASEAN, le Partenariat transpacifique et l'accord avec l'Australie encouragent aussi le recours à des guides régionaux pour élaborer les procédures d'évaluation de la conformité (par exemple, les Guides de l'ASEAN et de l'APEC). Le dernier accord, par exemple, appelle les parties à «affirmer leur intention d'adopter et d'appliquer les principes définis dans les Notes d'information de l'APEC sur les bonnes pratiques en matière de réglementation concernant les règlements techniques dans le cadre des procédures d'évaluation et d'approbation de la conformité, pour respecter les obligations internationales en conformité avec l'OMC » (article 7.3, accord SingapourAustralie). Les accords de l'ASEAN et l'accord avec les États-Unis encouragent la participation aux travaux des instances régionales de normalisation, comme le Comité consultatif sur les normes et la qualité de l'ASEAN (encadré 4) et le Programme de travail de l'APEC sur les normes et la conformité (encadré 11).

\section{Acceptation des règlements techniques comme équivalents}

87. Quatre accords (avec la Nouvelle-Zélande, l'Australie, l'Inde, le Panama) et le Partenariat économique stratégique transpacifique) incitent les parties à considérer comme équivalents d'autres règlements techniques et normes des parties. Une telle disposition figure aussi dans les accords favorisant l'alignement des règlements et normes sur les normes internationales (accord avec la Nouvelle-Zélande, l'Inde et Panama, et le Partenariat économique stratégique transpacifique). De plus, trois accords exigent des membres qu'ils expliquent sur demande le motif de non-équivalence à l'autre ou aux autres parties, ce qui va donc au-delà de l'Accord sur les OTC de l'OMC.

44. Il revient à chaque pays membre de mettre en œuvre les exigences relatives aux produits pharmaceutiques. 


\section{Encadré 11. Approche de I'APEC pour éliminer les OTC}

La Coopération économique Asie-Pacifique (APEC) est un forum visant à faciliter la croissance économique, la coopération, les échanges et l'investissement dans la région Asie-Pacifique. Elle a pour but de libéraliser les échanges et les investissements dans la région d'ici 2010 pour les économies industrialisées et d'ici 2020 pour les économies en développement (ces objectifs sont connus sous le nom de "Objectifs de Bogor ") ${ }^{1}$. L'APEC fonctionne selon le système des engagements non contraignants et du dialogue ouvert entre ses membres. Les décisions au sein de l'APEC sont adoptées par consensus et les engagements sont volontaires. Le Sous-comité sur les normes et la conformité de l'APEC, établi en novembre 1994, encourage la coopération entre les membres sur les normes et la conformité pour faciliter les échanges dans la région.

Le Sous-comité met en œuvre un plan de travail qui repose sur quatre piliers :

- $\quad$ Alignement des normes sur les normes internationales ;

- Reconnaissance des évaluations de la conformité dans des secteurs réglementés et facultatifs ;

- Transparence des normes et évaluation de la conformité dans les économies de l'APEC ; et

- Développement de l'infrastructure technique.

Les travaux d'harmonisation que I'APEC mène actuellement portent sur 7 domaines prioritaires (à savoir, le matériel et équipement électriques et électroniques, le matériel informatique, l'étiquetage des produits alimentaires, les produits en caoutchouc, les machines-outils et les normes et guides d'évaluation de la conformité). En 2005, 16 membres sur 21 avaient réalisé un alignement complet dans quatre domaines prioritaires, tandis que Singapour était parvenu à un alignement total dans tous les domaines prioritaires identifiés. En outre, deux ARM ont été conclus dans le cadre de "Pathfinder Initiatives " ${ }^{2}$ [initiatives pionnières] volontaires : l'Accord de reconnaissance mutuel de l'APEC relatif à l'évaluation de la conformité des équipements de télécommunication (APEC TEL MRA, 1998) et l'Accord de reconnaissance mutuelle de l'APEC relatif à l'évaluation de la conformité des équipements électriques et électroniques (APEC EE MRA, 1999). Singapour a adopté les deux accords.

Enfin, en novembre 2006, les ministres de l'APEC ont adopté cinq ensembles de « Mesures modèles pour les ACR/ALE ", dont un concernant les OTC. Les mesures modèles sont non contraignantes et ont pour but d'assurer une plus grande cohérence entre les ACR qui se recoupent et concernant les membres de l'APEC.

(1) 21 membres : Australie, Brunei Darussalam, Canada, Chili, Hong Kong, Chine, États-Unis, Indonésie, Japon, Malaisie, Mexique, Nouvelle-Zélande, Papouasie Nouvelle Guinée, Pérou, Philippines, République de Corée, République populaire de Chines, Russie, Singapour, Taipei chinois, Thaïlande, Vietnam

(2) Les « Pathfinder Initiatives » permettent aux membres de piloter la mise en œuvre d'initiatives de coopération avant leur adoption par les économies membres de l'APEC.

Source : OMC (2006a), http://www.apec.org

\section{Reconnaissance mutuelle des évaluations de la conformité}

88. Tous les accords examinés appellent les parties à reconnaître (mutuellement) les résultats des évaluations de la conformité menées par d'autres parties. Trois accords en particulier (avec la Corée, le Panama et le Partenariat économique stratégique transpacifique) précisent qu'il existe un large éventail de mécanismes pour faciliter l'acceptation des résultats de l'évaluation de la conformité, notamment :

- La confiance dans la déclaration de conformité d'un fournisseur.

- La reconnaissance unilatérale des résultats de l'évaluation de la conformité réalisée sur le territoire d'une autre partie.

- Les accords de coopération (volontaires) entre les instances d'évaluation de la conformité des territoires respectifs.

- La reconnaissance mutuelle des procédures d'évaluations de la conformité appliquées par les instances situées sur le territoire d'une autre partie.

- Des procédures d'accréditation pour les instances agréées d'évaluation de la conformité.

- Une désignation par les pouvoirs publics des instances d'évaluation de la conformité. 
89. Ces trois accords comportent aussi des dispositions relatives à l'enregistrement, la vérification et le suivi des instances d'évaluation de la conformité dans chaque partie, ce qui va donc au-delà des dispositions de l'OMC. De plus, la majorité des ACR examinés s'accompagnent d'accords distincts pour la reconnaissance mutuelle des procédures d'évaluation de la conformité ou bien encouragent la conclusion de tels accords (tableau 5). On peut dire de ces dispositions qu'elles renforcent l'Accord sur les OTC de l'OMC qui encourage d'autres consultations sur ces questions (article 6, Accord sur les OTC de l'OMC). Les membres de l'ASEAN, par exemple, ont conclu en 1998 un Accord cadre pour les ARM sectoriels et ils ont conclu depuis trois ARM de ce type, l'un pour les équipements de télécommunication (signé en 2001), l'autre pour les équipements électriques et électroniques (signé en 2002) et le dernier pour les produits cosmétiques (signé en 2003). Des ARM supplémentaires sont prévus pour les produits pharmaceutiques et les préparations alimentaires industrielles (OCDE, 2005). Parallèlement, des accords respectivement conclus avec les États-Unis et la Corée, appellent les parties à mettre en œuvre mutuellement la Phase I et la Phase II de l'Accord de reconnaissance mutuelle de l'APEC sur l'évaluation de la conformité des équipements de télécommunication ${ }^{45}$. Singapour a en fait conclu plusieurs ARM dans le domaine des équipements électriques et électroniques et des équipements de télécommunication, la plupart avec d'autres membres de l'APEC (l'exception étant l'Inde, voir tableau 5).

90. Enfin, seulement 2 accords examinés sur 10 exigent des parties qu'elles fournissent une explication en cas de non-reconnaissance des résultats ou des instances d'évaluation de la conformité, ce qui va donc au-delà de l'Accord de l'OMC (accord avec le Panama, et le Partenariat économique stratégique transpacifique).

Tableau 5. Vue d'ensemble des accords de reconnaissance mutuelle de Singapour, en avril 2007

\begin{tabular}{|c|c|c|c|c|c|}
\hline Accord & $\begin{array}{l}\text { Équipements } \\
\text { électriques et } \\
\text { électroniques }\end{array}$ & Cosmétiques & $\begin{array}{l}\text { Équipements de } \\
\text { télécommunication }\end{array}$ & $\begin{array}{c}\text { Produits } \\
\text { alimentaires } \\
\text { et horticoles }\end{array}$ & $\begin{array}{c}\text { Produits } \\
\text { pharmaceutiques }\end{array}$ \\
\hline ASEAN & 1 & 1 & $1^{* *}$ & & \\
\hline APEC & 1 & & $1^{* *}$ & & \\
\hline Singapour- Nouvelle Zélande & 1 & & & & \\
\hline Singapour- Japon & 1 & & & & \\
\hline Singapour-Australie & 1 & & 1 & 1 & 1 \\
\hline Singapour- États-Unis & & & 1 (APEC) & & \\
\hline Singapour-Canada* & & & 1 (APEC) & & \\
\hline Singapour-Taipei chinois* & & & 1 (APEC) & & \\
\hline Singapour-Hong Kong & & & 1 (APEC) & & \\
\hline Singapour-Malaisie & & & 1 (ASEAN) & & \\
\hline Singapour-Inde & $\begin{array}{l}1 \text { (pas encore } \\
\text { en application) }\end{array}$ & & $\begin{array}{c}1 \text { (pas encore en } \\
\text { application) }\end{array}$ & & \\
\hline
\end{tabular}

5 .

\section{Transparence}

91. Tous les ACR examinés comportent des dispositions sur la transparence imposant aux parties d'échanger des informations, de procéder à des consultations au niveau bilatéral ou régional et de notifier les règlements et les procédures d'évaluation de la conformité qui n'existaient pas, dont la teneur n'est pas

45. La Phase I se réfère à la reconnaissance mutuelle des rapports d'essais et la Phase II à la reconnaissance des certificats. 
conforme aux normes internationales ou qui peuvent entraver le commerce. La moitié des accords appelle explicitement à la mise en place d'un système bilatéral ou régional d'échange d'informations (par exemple un point de contact ou un point d'information dans chaque partie), tandis que les accords restants s'appuient sur les Points nationaux d'information concernant les obstacles techniques au commerce créés dans le cadre de l'Accord de l'OMC, les comités conjoints (voir ci-après) ou les secrétariats de groupements régionaux (par exemple le secrétariat de l'ASEAN). Aucun d'entre eux ne prévoit cependant, pour la présentation des observations, de délai plus rigoureux que la règle des « 60 jours au moins » de l'OMC.

6.

Mise en ouvre et règlement des différends

92. Tous les accords examinés appellent à la création d'un comité conjoint des obstacles techniques au commerce, pour contrôler et examiner la mise en œuvre des dispositions sur les OTC, suggérer des modifications, offrir une instance de consultation et de règlement de différends ou aider à déterminer les secteurs appelant une coopération plus poussée. Les membres de l'ASEAN ont par exemple établi des comités sectoriels conjoints pour les ARM. Autrement, les accords avec les États-Unis et le Panama n'exigent pas la création d'un Comité distinct des obstacles techniques au commerce, mais appellent les parties à nommer des coordinateurs « responsables de la coordination avec les parties intéressées pour toutes les questions concernant le renforcement de la coopération aux termes du chapitre [sur les obstacles techniques au commerce] » (article 2, accord Singapour-États-Unis). Par ailleurs, un certain nombre de comités ou d'organes ont pour mission de gérer une coopération plus poussée comme l'accréditation et l'enregistrement des organismes d'évaluation de la conformité (par exemple, le comité Singapour-Corée) ou l'harmonisation des normes (par exemple, le Comité consultatif sur les normes et la qualité de l'ASEAN et le Sous-comité sur les normes et la conformité de l'APEC).

93. Tous les accords examinés précisent comment résoudre les différends liés aux OTC entre les parties. Dans la plupart des cas, cela doit se passer au moyen de consultations au sein du Comité conjoint des obstacles techniques au commerce ${ }^{46}$. Si aucune solution ne peut être trouvée, quelques ACR recommandent de soumettre le différend à un mécanisme formel régional de règlement des différends (par exemple les accords de l'ASEAN et de l'Australie). Par contre, l'accord avec le Panama exclut le chapitre des obstacles techniques au commerce de la procédure régionale de règlement des différends, même si l'accord permet des consultations et des recommandations régionales à travers des groupes de travail ad hoc et des coordinateurs.

\section{Renforcement de la coopération}

94. Enfin, trois ACR sur dix comportent des dispositions spécifiques concernant l'assistance technique aux membres (accords avec l'Australie, l'Inde et entre les membres de l'ASEAN). L'Accord cadre de l'ASEAN pour les ARM sectoriels, par exemple, vise à fournir une formation aux pays en développement, améliorer leur infrastructure pour le calibrage, les essais, la certification et l'accréditation, afin de respecter les normes internationales correspondantes et soutenir l'instauration des institutions et du dispositif juridique nécessaire pour se conformer à l'Accord cadre (article 5, Accord cadre). De même, sur les 10 accords examinés, seule l'ASEAN prévoit une coopération dans le domaine de la métrologie, par exemple à travers une collaboration entre les instituts nationaux de métrologie et des membres et une participation active des membres aux accords conclus par les organismes régionaux et internationaux, comme le Programme de métrologie Asie-Pacifique, le Forum de métrologie légale Asie-Pacifique, le Bureau international des poids et mesures et l'Organisation internationale de métrologie légale.

46. L'accord Singapour-Japon, par exemple, signale que le Comité conjoint OTC « examinera les contestations dans les 20 jours» suivant leur notification. De même, le Comité OTC Singapour-AELE envisage d'« œuvrer à une solution convenable en conformité avec l'Accord sur les OTC de l'OMC » 
8. Conclusion

95. Tous les accords examinés renforcent et complètent l'Accord sur les OTC de l'OMC. Ils réaffirment tous les droits et les obligations des parties aux termes de l'Accord sur les OTC de l'OMC, la majorité appellent à un alignement des règlements techniques et des procédures d'évaluation de la conformité des parties sur les normes internationales et exigent des parties qu'elles reconnaissent mutuellement les résultats de leurs évaluations de la conformité, comme les y encourage l'Accord sur les OTC de l'OMC. Cela s'explique dans la plupart des cas par le niveau de développement comparable des parties et leur volonté de s'engager dans de larges partenariats économiques (par exemple, les accords avec la Nouvelle-Zélande, le Japon, l'Australie, les États-Unis, la Corée et le Partenariat économique transpacifique). Dans le cas de l'ASEAN et de l'APEC, le degré de libéralisation en matière d'OTC peut être surtout attribué au degré d'intégration que ces accords cherchent à favoriser. Deux accords encouragent par ailleurs le recours à des guides régionaux pour la mise en place de procédures d'évaluation de la conformité (par exemple, les recommandations de l'APEC), mais on peut dire que ces instructions aident les parties à respecter leurs obligations internationales selon les termes de l'OMC, et par conséquent ne sont pas en contradiction avec les règles multilatérales.

96. De plus, certains des ACR examinés comportent des dispositions qui vont au-delà des règles multilatérales. Environ un tiers des accords examinés, par exemple, impose aux parties d'expliquer aux autres parties les raisons de la non-équivalence des règlements techniques et de la non-reconnaissance des résultats des évaluations de la conformité. La même proportion comporte des dispositions sur l'enregistrement, la vérification et le suivi des instances d'évaluation de la conformité de chaque partie. On peut considérer que ces engagements, qui ont été pour la plupart inclus dans de récents accords (avec la Corée, l'Inde et le Panama, et le Partenariat économique stratégique transpacifique) renforcent la transparence, d'une part, et améliorent la coopération concernant la reconnaissance mutuelle des résultats de l'évaluation, d'autre part, et donc convergent vers l'Accord sur les OTC de l'OMC et le complètent. De même, Singapour a conclu, parallèlement à ses ACR, de nombreux accords de reconnaissance mutuelle des résultats de l'évaluation de la conformité (ARM) en particulier dans le domaine les équipements électriques, électroniques et de télécommunication. De tels accords vont au-delà des règles multilatérales, mais ils renforcent l'Accord sur les OTC de l'OMC qui encourage d'autres consultations sur ces questions.

\subsubsection{Dispositif institutionnel de gestion des OTC}

97. Le Standards, Productivity and Innovation Board (SPRING), conseil officiel établi sous l'égide du ministère du Commerce et de l'Industrie, est le principal organisme coordonnant la conception et la mise en œuvre de normes nationales, de règlements techniques obligatoires et de procédures d'évaluation de la conformité. Parmi les autres instances qui administrent les règlements techniques obligatoires figurent le Department of Industrial Safety (DIS), l'Infocomm Development Authority of Singapore (iDA), les ministères de la Santé et de l'Environnement, et le Public Utilities Board (OMC, 2004).

98. SPRING coordonne les efforts de normalisation de Singapour et administre le Consumer Protection (Safety Requirements) Registration Scheme dans le cadre duquel 45 catégories d'appareils ménagers électriques, électroniques et à gaz et accessoires sont enregistrées (l'enregistrement est obligatoire et est conditionné à la certification d'un organisme désigné d'évaluation de la conformité). Il intervient également comme autorité nationale sur la métrologie, les poids et mesures, et il est responsable de l'accréditation des organismes d'évaluation de la conformité ${ }^{47}$. SPRING est géré par un Directoire représentant le secteur, les syndicats et les pouvoirs publics et son Département de normalisation travaille

47. La mission de SPRING est en fait assez étendue et comprend l'amélioration des conditions pour les entreprises à Singapour, l'amélioration de la compétitivité des entreprises, l'accroissement de leurs possibilités et l'amélioration de leur accès aux marchés étrangers et de leurs opportunités. 
sous les instructions d'un conseil national de normalisation sectorielle, conseil lui-même à la tête de dix comités de normalisation chargés de formuler et d'établir des normes nationales dans neuf domaines définis (appareils électriques et électroniques, BTP, informatiques, services, produits chimiques, technologie médicale, ingénierie générale et sécurité, alimentation, systèmes de gestion) (site Internet de SPRING) ${ }^{48}$.

99. Les normes sont volontaires, sauf lorsque les instances de réglementation se réfèrent à la législation, auquel cas elles deviennent alors des règlements techniques obligatoires (par exemple, le Système d'enregistrement (des normes de sécurité) pour la protection des consommateurs). Elles sont préparées par les Comités de normalisation correspondants lorsqu'il paraît nécessaire d'améliorer les échanges commerciaux, la compétitivité, l'interopérabilité, la productivité, la qualité, l'environnement, la sécurité et la santé ${ }^{49}$. En principe, les normes internationales servent de base à la préparation des normes de Singapour sur les produits. SPRING précise que « la norme internationale sera adoptée intégralement et tout écart devra être convenablement justifié. En l'absence de norme internationale, les normes peuvent être alignées sur une norme nationale étrangère correspondante ». Un projet de normes est alors diffusé publiquement pour commentaires pendant une période de 60 jours avant d'être approuvé et publié au Journal officiel (OMC, 2004).

100. En 2005, environ $83 \%$ des normes nationales de Singapour étaient alignées sur les normes internationales (OCDE, 2005). SPRING est aussi membre de l'Organisation internationale de normalisation (ISO) et de la Commission électrotechnique internationale (CEI) et il participe activement aux instances régionales de normalisation et de conformité comme le Comité consultatif sur les normes et la qualité de l'ASEAN (ACCSQ), le Pacific Area Standards Congress (PASC), le Sous-comité sur les normes et la conformité de l'APEC (SCSC) et le Groupe de rencontre Asie-Europe (ASEM) sur les normes et la conformité. De plus, SPRING a accepté le Code de pratique pour l'élaboration, l'adoption et l'application des normes de l'OMC et publie l'état d'avancement de son programme de normalisation tous les six mois comme l'exige le Code (site Internet de SPRING).

101. Enfin, contrairement au Chili où toutes les informations sont centralisées, Singapour est doté de deux points d'information sur les règlements techniques et les normes (M. Low, ministre du Commerce et de l'Industrie, Singapour) :

- SPRING : pour les normes et les règlements techniques nationaux concernant des appareils et produits ménagers électriques, électroniques et à gaz, et des accessoires spécifiques conçus pour équiper des biens contrôlés. Les normes récemment adoptées sont publiées sur son site Internet et une liste complète est donnée dans son Catalogue de normes ${ }^{50}$.

- La Food Control Division in the Agro-Food and Veterinary Authority : traite des règlements techniques liés aux aliments industriels.

48. http://www.spring.gov.sg et http://www.standards.org.sg/.

49. En dehors des normes, SPRING met aussi au point des «références techniques », qui sont des documents traités par voie accélérée et conçus pour répondre aux demandes sectorielles de spécifications ou de prescriptions concernant un produit ou un processus particulier en l'absence de norme. Contrairement à une norme, une référence technique est émise sans avoir à passer par tout le processus de consensus. Elle est généralement évaluée dans un délai de deux ans ; SPRING décide alors si la référence technique doit être adoptée comme norme, retirée ou conservée comme référence technique, en fonction des réactions du secteur.

50. Pour plus de précisions sur les produits contrôlés, veuillez consulter www.spring.gov/sg/safety. Le site Internet de SPRING : http://www.standards.org.sg/. 


\section{3 $\quad$ Maroc $^{51}$}

\subsubsection{Introduction}

102. Le Maroc applique des ACR depuis la fin des années 80. Il a d'abord conclu de premiers accords commerciaux régionaux avec les pays voisins, devenant membre de l'Union du Maghreb arabe en 1989, et près de dix ans plus tard de la Zone de libre-échange panarabe (1998). Il a aussi conclu des accords bilatéraux de libre-échange avec la Tunisie, l'Égypte et la Jordanie (1999), avec lesquelles il s'est récemment engagé à instaurer une "Zone de libre-échange entre les pays méditerranéens arabes 》 (à travers l'Accord d'Agadir). Le Maroc a ensuite conclu des accords de libre-échange avec des pays européens (AELE en 1999 et un accord d'association économique avec l'UE en 2000), les Émirats arabes unis (2003) et plus récemment la Turquie et les États-Unis (2006). Le dernier Examen des politiques commerciales de l'OMC concernant le Maroc relève, cependant, que «le fait d'appartenir à plusieurs accords commerciaux dont la portée géographique, les programmes de libéralisation et les dispositions sur les règles d'origine (entre autres) diffèrent n'est pas seulement difficile à gérer, mais rend aussi le régime commercial marocain plus complexe » (OMC, 2003).

\subsubsection{Analyse comparative des dispositions régionales sur les OTC}

103. Le Maroc a pris des engagements en matière d'OTC aux termes de l'Accord sur les OTC de l'OMC, ainsi qu'aux termes de cinq ACR qui sont examinés dans cette partie (à savoir, les accords avec l'AELE, l'UE, les États-Unis et la Turquie, et l'Accord d'Agadir, voir tableau 6, lignes surlignées). En avril 2007, un ARM distinct, l'Accord sur l'évaluation de la conformité et l'acceptation des produits industriels, était en cours de négociation avec l'UE. Pour l'instant, le secteur électrique, les machines-outils et les produits de construction ont été identifiés comme des secteurs prioritaires (UE, 2006).

51. Mme Zitouni, Chef du service chargé de la normalisation et du contrôle de la qualité du ministère marocain de l'Industrie, du Commerce et de la mise à niveau de l'Économie, a fourni des informations précieuses pour cette étude de cas. 
Tableau 6. Vue d'ensemble des ACR du Maroc, en avril 2007

\begin{tabular}{|c|c|c|c|c|c|}
\hline $\mathbf{N}^{\circ}$ & Accord & $\begin{array}{l}\text { Entrée en } \\
\text { vigueur }\end{array}$ & Type d'accord & Notification à l'OMC & $\begin{array}{c}\text { Dispositions sur les } \\
\text { OTC }\end{array}$ \\
\hline 1 & Maroc-AELE & $1^{\text {er }}$ décembre 99 & $\begin{array}{l}\text { Accord de libre- } \\
\text { échange }\end{array}$ & oui & oui \\
\hline 2 & Maroc-UE* & $1^{\mathrm{er}}$ mars 00 & $\begin{array}{l}\text { Accord de libre- } \\
\text { échange }\end{array}$ & oui & oui \\
\hline 3 & $\begin{array}{l}\text { Maroc- États- } \\
\text { Unis*** }\end{array}$ & $1^{\mathrm{er}}$ janvier 06 & $\begin{array}{l}\text { Accord de libre- } \\
\text { échange }\end{array}$ & oui & oui \\
\hline 4 & Maroc- Turquie & $1^{\mathrm{er}}$ janvier 06 & $\begin{array}{l}\text { Accord de libre- } \\
\text { échange }\end{array}$ & oui & oui \\
\hline 5 & $\begin{array}{l}\text { Accord d'Agadir } \\
\text { (Maroc, Tunisie, } \\
\text { Égypte, Jordanie) }\end{array}$ & 27 mars 07 & $\begin{array}{l}\text { Accord de libre- } \\
\text { échange }\end{array}$ & non & oui \\
\hline 6 & $\begin{array}{l}\text { Union du Maghreb } \\
\text { arabe** } \\
\text { (Algérie, Libye, } \\
\text { Mauritanie, Maroc, } \\
\text { Tunisie) }\end{array}$ & 17 février 89 & $\begin{array}{l}\text { Accord de libre- } \\
\text { échange }\end{array}$ & non & s.o. \\
\hline 7 & $\begin{array}{l}\text { Zone de libre- } \\
\text { échange panarabe } \\
\text { ( } 22 \text { membres de la } \\
\text { Ligue arabe) }\end{array}$ & $1^{\mathrm{er}}$ janvier 98 & $\begin{array}{l}\text { Accord de libre- } \\
\text { échange }\end{array}$ & oui & non \\
\hline 8 & Maroc-Tunisie & 16 mars 99 & $\begin{array}{l}\text { Accord de libre- } \\
\text { échange }\end{array}$ & non & non \\
\hline 9 & Maroc-Égypte & 29 avril 99 & $\begin{array}{l}\text { Accord de libre- } \\
\text { échange }\end{array}$ & non & non \\
\hline 10 & Maroc-Jordanie & 21 octobre 99 & $\begin{array}{l}\text { Accord de libre- } \\
\text { échange }\end{array}$ & non & non \\
\hline 11 & $\begin{array}{l}\text { Maroc-Émirats } \\
\text { arabes unis** }\end{array}$ & 9 juillet 03 & $\begin{array}{l}\text { Accord de libre- } \\
\text { échange }\end{array}$ & non & s.o. \\
\hline
\end{tabular}

Source : Ministère du Commerce extérieur et Administration des douanes du Royaume du Maroc. http://www.ustr.gov

* Accord d'association économique EUROMED

** Accord confronté à d'importants problèmes de mise en œuvre (Oxford Business Group, 2005)

*** Le document lié à l'assistance technique en matière d'OTC est aussi analysé.

\section{Références à l'Accord sur les OTC de l'OMC}

104. Trois accords réaffirment les droits et obligations des parties aux termes de l'Accord sur les OTC de l'OMC (accords avec l'AELE, les États-Unis et la Turquie). Les accords récents conclus avec l'UE et les États-Unis visent à approfondir la coopération en matière de réglementation; en d'autres termes, les parties sont invitées à "définir des initiatives bilatérales de facilitation des échanges concernant les normes, règlements techniques et procédures d'évaluation de la conformité qui conviennent pour des problèmes ou secteurs spécifiques » (article 7.4, Accord États-Unis-Maroc).

\section{Harmonisation des règlements techniques, normes et évaluations de la conformité}

105. Le seul accord examiné imposant spécifiquement aux parties d'harmoniser leurs règlements techniques et procédures d'évaluation de la conformité est l'EUROMED. À travers cet accord, devraient être mis en œuvre « les moyens propres à promouvoir l'utilisation par le Maroc des règles techniques de la Communauté et des normes européennes relatives à la qualité des produits industriels et agroalimentaires, ainsi que les procédures de certification » (article 40). L'accord précise également que la coopération entre les deux parties " vise à aider le Maroc à rapprocher sa législation de celle de la Communauté » et de «développer l'utilisation des règles communautaires dans le domaine de la normalisation, de la métrologie, de la gestion et l'assurance de la qualité, et de l'évaluation de la conformité » (articles 51 et 52). Les autres accords examinés encouragent une «plus grande coopération » dans le domaine des règlements techniques, normes et évaluations de la conformité, sans préciser quelle forme cette coopération devrait 
prendre (harmonisation, équivalence, reconnaissance mutuelle, etc.). L'accord avec l'AELE et l'accord avec la Turquie cependant encouragent l'un et l'autre la coopération à travers l'utilisation de "solutions adoptées à l'échelle européenne » (étant donné que leurs règles, normes et procédures d'évaluation de la conformité sont aussi censées être alignés sur les réglementations de l'UE), tandis que l'Accord d'Agadir et l'Accord États-Unis-Maroc encouragent une coopération sur la base des normes internationales. Aucun des accords examinés n'impose aux membres d'accepter l'équivalence des règlements techniques et normes d'autres membres (bien qu'une telle option soit encouragée dans l'Accord États-Unis-Maroc, parallèlement à d'autres options).

\section{Reconnaissance mutuelle des évaluations de la conformité}

106. Les accords avec l'UE et les États-Unis encouragent la reconnaissance (mutuelle) des résultats des évaluations de la conformité à travers la conclusion d'un ARM. Le Maroc a engagé des discussions avec l'UE à cet égard mais, à ce jour, n'a pas encore conclu d'ARM ${ }^{52}$. Les dispositions sur la reconnaissance contenues dans l'accord avec les États-Unis vont au-delà des règles de l'OMC, en exigeant des parties qu'elles expliquent, sur demande, la non-reconnaissance des résultats des évaluations de la conformité et la non-accréditation des instances d'évaluation de la conformité. En outre, l'accord de libreéchange entre les États-Unis et le Maroc précise que lorsqu'une partie refuse une demande d'engager des négociations ou de conclure un ARM, « elle doit, à la demande de l'autre partie, expliquer les raisons de sa décision » (article 7.5).

5.

Transparence

107. Trois des cinq ACR examinés (accords avec l'AELE, les États-Unis et la Turquie) comportent des dispositions sur la transparence qui exigent des parties qu'elles échangent des informations, organisent des consultations et notifient et publient les règlements techniques et les procédures d'évaluation de la conformité, conformément aux règles de l'Accord sur les OTC de l'OMC. Aucun de ces accords n'exige des parties qu'elles créent des points d'information distincts au niveau bilatéral ou régional, ou qu'elles ménagent un délai de présentation des observations qui soit plus long que celui stipulé par le Comité des obstacles techniques au commerce de l'OMC («60 jours au moins »). En outre, l'accord États-Unis-Maroc exige que chaque partie autorise les personnes de l'autre partie à participer à la conception de normes, de règlements techniques et de procédures d'évaluation de la conformité à des conditions qui ne sont pas moins favorables que celles accordées à ses propres personnes (article 4.6; Accord États-Unis-Chili). Une telle disposition va au-delà de l'Accord sur les OTC de l'OMC qui ne fait qu' "encourager» une coopération de ce type.

6.

Mise en œuvre et règlement des différends

108. Tous les accords examinés (en dehors de l'EUROMED) prévoient des consultations et des recommandations entre les parties pour résoudre les différends liés aux OTC. L'Accord d'Agadir, par exemple, énonce que «si un des États membres a recours à des procédures qui créent [...] des obstacles techniques, les États membres tiendront aussitôt des délibérations afin de trouver des solutions adaptées » (article 23). Cependant, aucun des ACR, à l'exception de celui avec les États-Unis, n'exige des parties qu'elles créent une instance dédiée aux OTC ou désignent un coordinateur au niveau bilatéral ou régional, chargé de contrôler la mise en œuvre des dispositions sur les OTC et de gérer les consultations. L'Accord États-Unis-Maroc exige que les Coordinateurs OTC des parties assurent le suivi de la mise en œuvre des dispositions, gèrent une coopération plus poussée et aident à mener des consultations et résoudre les

52. Un ARM avec l'UE reposerait sur l'adoption des règlements techniques et des procédures d'évaluation de la conformité de l'UE dans certains secteurs donnés. Source : informations fournies par Mme Zitouni, ministère marocain de l'Industrie et du Commerce. 
différends en rapport avec les OTC. Si une solution ne peut être trouvée, l'Accord autorise les parties à recourir à la procédure de règlement des différends établie aux termes de l'accord (des dispositions semblables sur le règlement des différends figurent dans les accords avec l'AELE et la Turquie).

\section{Renforcement de la coopération}

109. Enfin, les accords avec les États-Unis et l'UE comportent des dispositions spécifiques sur l'assistance technique, destinées à renforcer l'infrastructure institutionnelle, juridique et physique du Maroc pour les problèmes liés aux OTC, afin de faciliter le respect par le Maroc des dispositions sur les OTC contenues dans l'EUROMED et l'accord de libre-échange entre les États-Unis et le Maroc (encadré 12). En outre, le partenariat EUROMED engage les parties à coopérer dans le domaine de la métrologie.

\section{Encadré 12. Assistance liée aux OTC aux termes de l'Accord de libre-échange entre les États-Unis et le Maroc}

Dans le cadre de l'Accord de libre-échange entre les États-Unis et le Maroc, les États-Unis se sont engagés à aider le Maroc à mettre en œuvre le chapitre sur les OTC de cet Accord et à améliorer sa capacité à concevoir, mettre en œuvre et publier des règlements techniques, normes et procédures d'évaluation de la conformité. Une lettre jointe à l'Accord contient des engagements spécifiques d'assistance technique et de renforcement des capacités institutionnelles), qui sont les suivants :

- $\quad$ Aider le Maroc à renforcer son système de réglementation et à améliorer les capacités de réglementation d'entités qui mettent au point et notifient les règlements techniques et traitent les demandes d'information du public qui s'y rapportent.

- Proposer une formation à des agents publics marocains pour améliorer la mise au point de normes et de certification et accréditation des organismes d'évaluation de la conformité, et approfondir les connaissances des principaux responsables concernant le système américain dans les secteurs d'exportation présentant un intérêt pour le Maroc.

- Encourager les liens et les relations de coopération entre les instances gouvernementales et nongouvernementales qui mettent au point des normes et évaluent la conformité aux normes.

- Éduquer les entreprises de chacune des parties relativement aux règlements techniques, normes et procédures d'évaluation de la conformité de l'autre pays, au moyen de séminaires et en utilisant les ressources sur l'Internet.

- Éduquer les entreprises marocaines des secteurs présentant un intérêt particulier, comme le textile et le cuir, les pièces détachées automobiles, l'électronique et les aliments industriels, quant aux approches réglementaires adoptées par les États-Unis dans ces secteurs et aux méthodes à suivre pour satisfaire aux prescriptions des règlements techniques des États-Unis.

Source : USTR, 2004.

\section{8. $\quad$ Conclusion}

110. La majorité des ACR du Maroc réaffirment les droits et obligations des parties aux termes de l'Accord sur les OTC de l'OMC et exigent des parties qu'elles "coopèrent» dans le domaine des règlements techniques, normes et évaluations de la conformité. Cela étant, les modalités de fonctionnement d'une telle coopération restent souvent vagues (le texte est souvent moins précis que l'Accord sur les OTC de l'OMC). Bien que les trois accords favorisent l'harmonisation par rapport aux normes de l'UE, les deux autres ACR examinés encouragent l'alignement sur les normes internationales. Des contradictions dans la stratégie d'harmonisation du Maroc pourraient en principe se faire jour dans des cas où les normes de l'UE s'écartent nettement des normes et pratiques internationales. De plus, aux termes de l'accord entre les ÉtatsUnis et le Maroc, les personnes morales américaines sont autorisées à participer à l'élaboration des normes, règlements techniques et évaluations de la conformité du Maroc, ce qui pourrait compliquer le processus d'alignement sur les normes de l'UE. 
111. En termes de transparence et de reconnaissance (mutuelle) des procédures d'évaluation de la conformité également, aucun des ACR, à l'exception de l'accord avec les États-Unis, ne va au-delà des dispositions de l'OMC. En outre, bien qu'une majorité des ACR prévoit des consultations dans le cas où une partie considère que l'autre partie a pris des mesures qui risquent de créer, ou ont créé, un obstacle technique au commerce, seul un accord (avec les États-Unis) établit un mécanisme distinct pour contrôler la mise en œuvre des obligations bilatérales liées aux OTC.

112. Le manque d'obligations précises pour libéraliser les OTC (que ce soit au moyen d'une harmonisation, d'une équivalence ou d'une reconnaissance mutuelle des règlements techniques, normes et procédures d'évaluation de la conformité) qui prédomine dans la majorité des ACR examinés, l'absence d'ARM et la faible «institutionnalisation » (autrement dit, la carence des mécanismes de contrôle) peut s'expliquer par les capacités relativement limitées du Maroc en matière d'institutions et d'infrastructure. Le Maroc a cependant engagé une réforme complète de son système de réglementation de la normalisation et de l'accréditation, et il bénéficie d'une assistance technique de l'UE et des États-Unis pour moderniser ses dispositifs et son infrastructure liés aux normes.

\subsubsection{Dispositif institutionnel de gestion des OTC}

113. Les normes et les spécifications techniques sont élaborées par les comités techniques mis en place par les ministères responsables des produits auxquels ces normes doivent s'appliquer. Elles se fondent en principe sur des normes internationales, notamment celles de l'Organisation internationale de normalisation (ISO), de l'association française de normalisation (AFNOR) et le recueil de normes alimentaires internationales qu'est le Codex Alimentarius FAO/OMS (OMC, 2003). Une fois approuvées, elles font l'objet d'une publication au journal officiel.

114. Les normes et les spécifications techniques marocaines sont généralement optionnelles, mais elles peuvent être rendues obligatoires par arrêté du ou des ministres concernés, principalement pour des raisons liées à la protection de la santé, de la sécurité, de l'hygiène et de l'environnement. Elles deviennent alors obligatoires et s'appliquent sans discrimination aux produits importés et aux produits locaux. Des règlements techniques s'appliquent à l'heure actuelle à certains produits en fer et en acier, produits gaziers, équipements électriques, produits textiles, appareils ménagers et jouets (OMC, 2003). Le Service de normalisation industrielle marocaine (SNIMA), rattaché au ministère de l'Industrie et du Commerce, publie et promeut les normes et règlements techniques et sert de point d'information du Maroc (http://www.mcinet.gov.ma/snima/). En juillet 1997, le SNIMA a officiellement adopté le Code de pratique pour l'élaboration, l'adoption et l'application des normes (OMC, 2003). Le Maroc a également mis en place un système de certification des entreprises reposant sur les normes ISO 9000 et ISO 14000 ainsi qu'un dispositif d'accréditation des laboratoires d'essais et de calibrage conforme aux critères du guide international ISO/CEI 58. Les accréditations sont accordées sur la base des normes marocaines.

115. Le Maroc réexamine actuellement son cadre réglementaire en matière de normalisation, de certification et d'accréditation (Encadré 13). Cette réforme vise à :

- renforcer l'implication du secteur privé dans l'élaboration et l'application des mesures à caractère normatif,

- $\quad$ soutenir davantage les organismes d'évaluation de la conformité en vue de promouvoir la négociation d'ARM internationaux,

- améliorer la gestion des activités d'harmonisation, de certification et d'accréditation, notamment dans le sens d'une plus grande transparence et d'une communication accrue sur les problèmes liés aux normes (SNIMA, 2007). 
TAD/TC/WP(2007)12/FINAL

\section{Encadré 13. Réforme du système marocain de normalisation}

Le Maroc prévoit de mettre en place trois nouvelles institutions chargées des questions relatives aux normes :

- Le Comité Marocain d'Accréditation (CMA) : qui sera chargé de l'évaluation des compétences des organismes de certification, d'essais et de qualification.

- $\quad$ L'Institut marocain de normalisation (IMANOR) : qui sera créé et placé sous la tutelle du Ministre chargé de l'Industrie, sera composé de plusieurs sous-commissions techniques chargées d'élaborer les normes et règlements techniques dans leur domaine de compétence respectif. II coordonnera les activités liées à l'élaboration et à la publication de normes et règlements techniques et à la délivrance des certificats de conformité. II contribuera en outre à aligner les normes et règlements marocains sur les normes et règlements internationaux à chaque fois que cela sera nécessaire, favorisera la conclusion d'ARM et représentera le Maroc lors des travaux de normalisation régionaux et internationaux.

- Le Conseil Supérieur de Normalisation, de Certification et d'Accréditation (CSNCA), qui sera créé auprès du Premier ministre, sera composé de représentants du secteur public et du secteur privé. II définira les orientations stratégiques du programme général des travaux de normalisation et travaillera en coordination étroite avec l'Institut marocain de normalisation. 


\section{CONCLUSIONS PRINCIPALES ET RECOMMANDATIONS}

116. En conclusion, la plupart des règles bilatérales et régionales relatives aux OTC tendent à converger vers le système commercial multilatéral et à le conforter. Les accords qui visent une intégration économique plus poussée (allant au-delà du libre-échange) et qui affichent des caractéristiques allant audelà des règles de l'OMC, en particulier, peuvent venir compléter et renforcer l'Accord sur les OTC de l'OMC. Lorsqu'ils sont effectivement mis en œuvre, ces accords peuvent faciliter la coopération réglementaire entre les parties et leur permettre de mettre en place une coordination plus étroite et plus rapide sur les mesures à caractère normatif que cela ne pourrait être le cas à l'échelon multilatéral. En outre, une meilleure harmonisation et une plus grande transparence de ces mesures entre les parties peuvent faciliter l'accès des tiers au marché (ceux-ci n'étant dès lors tenus de se conformer qu'à un ensemble unique de mesures lorsqu'ils s'implantent sur les marchés couverts par l'ACR) et leur offrent la possibilité d'être mieux informés des exigences réglementaires. Enfin, la mise en œuvre effective de ces ACR peut améliorer les pratiques et l'infrastructure réglementaire des (pays en développement qui y sont) parties, favoriser une plus grande communauté de vues des parties sur les problèmes internationaux relatifs aux OTC et renforcer leur pouvoir de négociation collectif au sein des instances internationales. Ces éléments peuvent, à leur tour, permettre une meilleure application de l'Accord sur les OTC de l'OMC et encourager la réduction des OTC à l'échelon multilatéral.

117. Cela étant, les ACR et les ARM sont discriminatoires par essence et peuvent parfois représenter des obstacles pour le système commercial multilatéral. Lorsque les règlements, procédures et normes régionaux en application dans un pays ou une région divergent substantiellement des règlements, procédures et normes internationaux ou que des initiatives bilatérales ou régionales sont menées indépendamment des efforts internationaux accomplis et détournent l'attention des négociations multilatérales engagées, ils peuvent représenter des contraintes supplémentaires pour les régulateurs, qui doivent alors investir plus de temps et de ressources dans une multitude de procédures parallèles ainsi que pour les entreprises, qui doivent se conformer à différents référentiels de normes sur les produits et procédures d'évaluation de la conformité. Ces contraintes sont encore plus importantes pour les pays pauvres confrontés à des problèmes de capacités d'ordre administratif et technique.

118. Un certain nombre d'approches pourraient être adoptées dans les ACR pour traiter ces problèmes et faire en sorte que ces accords viennent renforcer le système commercial multilatéral et favorisent une plus grande cohésion des règles bilatérales, régionales et internationales visant à éliminer les OTC :

- Recommandation 1: Apporter une assistance effective aux pays à faible revenu. Outre le fait d'inciter les parties à participer aux négociations commerciales et aux processus de normalisation régionaux et internationaux, les ACR devraient aussi les encourager, si on leur en fait la demande, à fournir des formations aux représentants des pouvoirs publics, autorités de tutelles et associations professionnelles des parties moins développées afin qu'ils soient mieux informés et aient une meilleure connaissance des questions relatives aux OTC à l'échelon régional et international et soient en mesure de mieux évaluer les coûts et avantages des différentes options d'action publique visant à réduire les OTC. De plus, les mécanismes régionaux de consultation et de suivi devraient être renforcés si besoin, afin que les engagements pris à l'échelon régional en matière d'OTC puissent être mieux respectés. Enfin, les principales infrastructures et institutions relatives aux OTC devraient être consolidées pour renforcer la confiance dans la fiabilité des procédures et dispositifs des pays en développement (par exemple les organismes chargés des essais, du contrôle de la qualité, de l'évaluation de la conformité et de l'accréditation).

- Recommandation 2 : Renforcer la transparence et la disponibilité des informations relatives aux efforts accomplis à l'échelon bilatéral et régional en matière d'OTC et des mesures à caractère normatif, obligatoires ou volontaires, en vigueur dans les pays parties aux ACR, pour éviter une redondance des efforts menés et informer les pays tiers. Les parties aux ACR devraient s'attacher à 
diffuser davantage d'informations relatives au respect des obligations qui y sont contenues. De plus, les points d'information devraient être incités à rassembler et à diffuser (à titre gracieux) toutes les informations utiles concernant les OTC à l'échelon bilatéral ou régional, par l'intermédiaire d'un site Internet par exemple. Parallèlement, il convient de poursuivre les efforts menés à l'échelle internationale pour améliorer la collecte des données et la transparence des mesures relatives aux OTC en cours de négociation ou en vigueur dans différents pays/différentes régions (par l'entremise du Comité de l'OMC sur les OTC et du Comité de l'OMC sur les accords commerciaux régionaux, par exemple).

- Recommandation 3 : Encourager l'adoption de «dispositions types " dans les chapitres relatifs aux OTC des ACR afin d'éviter toute contradiction entre ces accords et d'assurer leur cohésion avec le système commercial multilatéral. Les récents efforts accomplis par la Coopération économique Asie-Pacifique (APEC) pour rationaliser et simplifier les dispositions relatives aux OTC des différents accords bilatéraux et régionaux constituent un bon exemple à cet égard. Idéalement, l'élaboration des chapitres sur les OTC contenus dans les ACR devrait être le fruit d'une étroite coordination à l'échelon national et d'un dialogue entre les négociateurs des ACR, les experts de l'OMC et les autorités de tutelle et associations professionnelles nationales œuvrant à l'élaboration, à l'application et à la mise en œuvre des règlements techniques, des évaluations de la conformité et des normes volontaires à l'échelon national, régional et international. Pour assurer cette convergence et renforcer le système commercial multilatéral, les ACR devraient systématiquement :

1. réaffirmer les droits et obligations des parties aux termes de l'Accord sur les OTC de l'OMC, ainsi que les objectifs visés par cet accord.

2. imposer que les normes et guides internationaux (lorsqu'ils existent) servent de base pour élaborer des règlements et procédures d'évaluation de la conformité nationaux et régionaux. Les ACR pourraient indiquer aux parties comment appliquer au mieux ces normes internationales aux règlements techniques et procédures d'évaluation de la conformité nationaux (en tenant compte de leur niveau de développement et de leurs priorités).

3. encourager une convergence de vues entre les parties aux ARC sur les questions internationales en matière d'OTC afin de leur donner davantage "voix» et de renforcer leur pouvoir de négociation au sein des instances internationales.

4. promouvoir effectivement une coordination et une coopération plus intenses en matière de réglementation entre les parties aux ACR en vue de favoriser la réduction des OTC à l'échelon régional et multilatéral et d'encourager l'adoption de «bonnes pratiques» réglementaires par celles-ci.

- Recommandation 4 : Chercher à «internationaliser " les initiatives régionales mises en auvre avec succès dans certains secteurs prioritaires. Dans les secteurs mondialement intégrés se caractérisant par une part élevée d'échanges intra-sectoriels, comme les équipements de télécommunications et d'informatique, il faudrait idéalement et à chaque fois que cela est possible, que les ARM aient une portée non seulement régionale mais aussi internationale. Cela risque cependant d'être difficile en raison des différences de niveau de développement et de capacités entre les pays. Le document APEC (2006b) souligne néanmoins, à cet égard, l'impact favorable que peuvent avoir les accords multilatéraux conclus entre organismes d'évaluation de la conformité du secteur (privé) à caractère volontaire. Il relève que ces initiatives ont réussi à réduire les OTC pour les produits réglementés, grâce à la confiance mutuelle des parties dans les processus d'évaluation de leurs pairs. 


\section{RÉFÉRENCES}

APEC (2006a), « Report on the APEC International Symposium: Catalyc Role of the APEC Process: Behind the Border, Beyond the Bogor Goals », document présenté par le Japon au Comité de l'APEC sur le commerce et l'investissement, réunion des 25 et 26 mai 2006.

APEC (2006b), « An Effective Role for APEC in Addressing Technical Barriers to Trade », document présenté par la Nouvelle-Zélande et Singapour au Comité de l'APEC sur le commerce et l'investissement, réunion des 25 et 26 mai 2006.

ASEAN (2005), ASEAN Policy Guidelines on Standards and Conformance, Secrétariat de l'ASEAN, Djakarta.

Asia-Pacific Co-operation (Coopération économique Asie-Pacifique ou APEC) (2006), « Report on Case Study to Clarify the Effectiveness of Mutual Recognition Arrangements (MRAs) », document présenté par le Japon au Sous-comité sur les normes et la conformité de l'ASEAN, réunion des 8 et 9 septembre 2006.

Association of South-East Asian Nations (Association des nations de l'Asie du Sud-Est ou ASEAN) (2003), Work Programme of ASEAN Consultative Committee on Standards and Quality, Secrétariat de l'ASEAN, Djakarta.

Baldwin, R.E., (2000), Regulatory Protectionism, Developing nations and a Two-Tier World Trade System, Centre for Economic Policy Research (CEPR) Discussion Paper No. 2574, CEPR, Londres.

Baldwin, R.E., (2004), Stepping Stones or Building Blocks? Regional and Multilateral Integration, NCCR, Berne.

Baller, S., (2007), Trade Effects of Regional Standards Liberalisation: A Heterogeneous Firm Approach, Banque mondiale, Washington D.C.

Banque mondiale (2003), Standards and Global Trade: A Voice for Africa, Banque mondiale, Washington.

Banque mondiale (2005), Global Economic Prospects: Trade, Regionalism and Development, Banque mondiale, Washington D.C.

Casella, A. (1997), « Free Trade and Evolving Standards », dans J. Bhagwati et R.E. Hudec (eds.), Fair Trade and Harmonisation. Pre-requisites for Free Trade, Vol. 1, MIT Press, Cambridge, Massachusetts

CE (2001), Mise en œuvre de la politique du commerce extérieur dans les domaines des normes et de l'évaluation de la conformité : Un arsenal d'instruments, Document de travail des services de la Commission, CE, Bruxelles.

CE (2004), Priorités pour les activités relatives au commerce Bilatéral/Régional dans le domaine des accords de reconnaissance mutuelle pour les produits industriels et le dialogue technique relatif, Document de travail des services de la Commission, CE, Bruxelles.

CE (2006), Mutual Recognition Agreements Newsletter No.1, février 2006, CE, Bruxelles. 
Centre européen de gestion des politiques de développement (European Centre for Development Policy Management ou ECDPM) (à paraître), InBrief6, Comparing EU Free Trade Agreements: Technical Barriers to Trade, ECDPM, Maastricht.

Commission européenne (CE) (1996), La politique extérieure de la Communauté dans le domaine des normes et de l'évaluation de la conformité, Communication de la Commission, CE, Bruxelles.

Conférence des Nations Unies sur le commerce et le développement (CNUCED), (2005), Le multilatéralisme et le régionalisme: la nouvelle interface, $\mathrm{ONU}$, Genève.

Crawford, J.A., et Fiorentino, R.V. (2005), The Changing Landscape of Regional Trade Agreements, $\mathrm{OMC}$, Genève.

Czubala, W. et. al. (2007), Help or Hindrance? The Impact of Harmonised Standards on African Exports, Version préliminaire, Banque mondiale, Washington D.C.

Foss, I., (2003), « The Value of ARSO: Evaluation of the Future Development of the African Regional Organisation for Standardisation », document préparé pour l'Atelier stratégique de l'ORAN parrainé par le Sida (Suède), du 9 au 10 février 2004.

Hoekman et Kostecki (2001), The Political Economy of the World Trading System, Second Edition, Oxford, Royaume-Uni.

Kotschwar, B.F. (2001), «Standards and Technical Barriers to Trade », dans Toward Free Trade in the Americas, Brookings Institution Press (OEA), Washington, pp. 141 à 162.

Lesher, M. et S. Miroudot, « Analysis of the Economic Impact of Investment Provisions in Regional Trade Agreements », Documents de travail de l'OCDE sur la politique commerciale $n^{\circ} 36$, OCDE, Paris.

Mercado Común del Sur (MERCOSUR) (2002), Resolution of the Common Market Group, MERCOSUR/GMC/RES No. 02/9.

Nielson, J. (2003), Circulation des prestataires de services: accords de reconnaissance mutuelle, OCDE, Paris

OCDE (2005), Atelier sur les « Normes et évaluation de la conformité dans les échanges: minimiser les obstacles, optimiser les avantages » (organisé conjointement avec InWEnt gGmbH), Berlin, 21 et 22 novembre 2005, OCDE, Paris.

OCDE (2005a), Études de l'OCDE sur la politique commerciale - Au-delà des tarifs: Le rôle des obstacles non tarifaires dans le commerce international, OCDE, Paris

OMC (1996), Mise en æuvre et administration de l'Accord sur les obstacles techniques au commerce Communication de Singapour, G/TBT/2/Add.25.

OMC (2002), Décisions et recommandations adoptées par le Comité depuis le ler janvier 1995, G/TBT/1/Rev.8.

OMC (2003), Examen des politiques commerciales: Chili, OMC, Genève.

OMC (2003a), Examen des politiques commerciales: Maroc, OMC, Genève.

OMC (2004), Examen des politiques commerciales: Singapour, OMC, Genève. 
OMC (2006), Mise en æuvre et administration de l'Accord sur les obstacles techniques au commerce Communication du Chili, G/TBT/Add.16/Rev.1, OMC, Genève.

OMC (2006a), Rapport sur les résultats des travaux d'alignement des normes dans le cadre de l'APEC, G/TBT/W/262, Genève.

OMC (2007), Douzième examen annuel de la mise en œuvre et du fonctionnement de l'Accord OTC, Note du Secrétariat, G/TBT/21/Rev.1.

Organisation de coopération et de développement économiques (OCDE) (2003), Le régionalisme et le système commercial multilatéral, OCDE, Paris.

Organisation des États américains (OEA) (1998), Provisions on Standards and Conformity Assessment in Trade and Integration Arrangements of the Western Hemisphere, SG/TU/WG.SOTC/DOC6/96/Rev.3, OEA, Washington D.C.

Organisation mondiale du commerce (OMC) (1995), Accord sur les obstacles techniques au commerce, $\mathrm{OMC}$, Genève

Oxford Business Group (2005), Morocco-UAE: What happened to the FTAs?, dans l'édition du 4 décembre 2005 du Morocco Times, postée sur le site Internet : http://www.bilaterals.org

Piermartini, R. et M. Budetta (2006), « A Mapping of Regional Rules on Technical Barriers to Trade », Note préparée pour la réunion sur les règles régionales dans le système commercial mondial de la Banque interaméricaine de développement et de la Banque mondiale, qui s'est tenue à Washington, les 26 et 27 juillet 2006 .

Poncin, M.C (2002), Mutual Recognition Agreements: Their Role Today and Tomorrow, note présentée lors de l'Atelier de la CEE-ONU sur les questions relatives à la normalisation et à l'évaluation de la conformité dans les économies en transition (Workshop on Standardization and Conformity Assessment Matters in the Transition Economies), qui a eu lieu les 12 et 13 décembre 2001 à Bratislava.

Sampson, G.P. et S. Woolcock (2003), Regionalism, Multilateralism, and Economic Integration: The Recent Experience, ONU, New York.

Sen, R. (2006), «'New Regionalism' in Asia: A Comparative Analysis of Emerging Regional and Bilateral Trading Agreements involving ASEAN, China and India », Journal of World Trade, 40(4.), pp. 553 à 596.

Shortall, D. (2006), Document de travail de l'OCDE sur la politique commerciale $n^{\circ} 48$, « Regulatory Reform and Market Openness: Processes to Assess Effectively the Trade and Investment Impact of Regulation », OCDE, Paris.

Solano, O. et A. Sennekamp (2006), « Dispositions relatives à la concurrence contenues dans les accords commerciaux régionaux », Document de travail de l'OCDE sur la politique commerciale $n^{\circ} 31$, OCDE, Paris.

Vallina, A.M. (2006), « Good Regulatory Practices: The Chilean Case », présentation préparée par Ana Maria Vallina.

Wilson, J.S. et T. Otsuki (2004), Standards and Technical Regulations and Firms in Developing Countries: New Evidence from a World Bank Technical Barriers to Trade Survey, Banque mondiale, Washington D.C. 
Bases de données et sites Internet contenant les textes légaux des accords commerciaux bilatéraux et régionaux :

$\mathrm{OMC}$ - Accords commerciaux régionaux - Portail

http://www.wto.org/french/tratop_f/region_f/region_f.htm

SICE Foreign Trade Information System. TBT Portal : http://www.sice.oas.org

World Trade Law FTA Database, http://www.worldtradelaw.net/fta/ftadatabase/ftas.asp.

CIB Database, Tuck Business School,

http://cibresearch.tuck.dartmouth.edu/trade_agreements_db/database_home.php.

Site Internet de la Communauté andine, http://www.communidadandina.org

Direction générale des affaires économiques internationales du Chili, (Direcon, 2007): http://www.direcon.cl

International Enterprise Singapore (2007), http://www.iesingapore.gov.sg/

Ministère du commerce extérieur du Royaume du Maroc :

http://www.mce.gov.ma/AccordConv/Accord_Lib.asp

Administration des douanes du Royaume du Maroc : http://www.douane.gov.ma/

Service de Normalisation Industrielle Marocaine (SNIMA) (2007), http://www.mcinet.gov.ma/snima/. 


\section{ANNEXE I : VUE D'ENSEMBLE DES ACR EXAMINÉS}

Tableau 6. Liste des ACR examinés

\begin{tabular}{|c|c|c|c|c|c|}
\hline Accord & $\begin{array}{l}\text { Date d'entrée } \\
\text { en vigueur }\end{array}$ & membres & Type & $\begin{array}{l}\text { Chapitre sur les } \\
\text { OTC }\end{array}$ & Examiné par \\
\hline $\begin{array}{l}\text { Accord de libre- } \\
\text { échange de l'ASEAN } \\
\text { (AFTA) }\end{array}$ & 28 janvier 92 & $\begin{array}{c}\text { Brunei, Cambodge, Indonésie, } \\
\text { Laos, Malaisie, Birmanie, } \\
\text { Philippines, Singapour, Thaïlande, } \\
\text { Vietnam }\end{array}$ & Accord préférentiel & oui & $\begin{array}{l}\text { auteur } \\
\text { Piermartini et Budetta } \\
\text { (2006) }\end{array}$ \\
\hline $\begin{array}{l}\text { Communauté andine } \\
\text { (CAN) }\end{array}$ & 25 mai 88 & $\begin{array}{l}\text { Bolivie, Colombie, Equateur, } \\
\text { Pérou }\end{array}$ & Accord préférentiel & oui & $\begin{array}{l}\text { Piermartini et Budetta } \\
\text { (2006) } \\
\text { Kotschwar (2001) }\end{array}$ \\
\hline $\begin{array}{l}\text { Accord commercial } \\
\text { dans le cadre de } \\
\text { relations } \\
\text { économiques plus } \\
\text { étroites entre } \\
\text { l'Australie et la } \\
\text { Nouvelle-Zélande } \\
\text { (CER) }\end{array}$ & 1er janvier 83 & Australie-Nouvelle-Zélande & $\begin{array}{l}\text { Accord de libre- } \\
\text { échange }\end{array}$ & oui & $\begin{array}{l}\text { Piermartini et Budetta } \\
\text { (2006) }\end{array}$ \\
\hline Australie -Thaïlande & non ratifié & & $\begin{array}{l}\text { Accord de libre- } \\
\text { échange }\end{array}$ & oui & $\begin{array}{l}\text { Piermartini et Budetta } \\
\text { (2006) }\end{array}$ \\
\hline Australie - États-Unis & 1er janvier 05 & & $\begin{array}{l}\text { Accord de libre- } \\
\text { échange }\end{array}$ & oui & $\begin{array}{l}\text { Piermartini et Budetta } \\
\text { (2006) }\end{array}$ \\
\hline $\begin{array}{l}\text { Marché commun } \\
\text { centroaméricain } \\
\text { (CACM) }\end{array}$ & 12 octobre 61 & $\begin{array}{c}\text { Costa Rica, El Salvador, } \\
\text { Guatemala, Honduras, Nicaragua }\end{array}$ & Union douanière & oui & $\begin{array}{l}\text { Piermartini et Budetta } \\
(2006) \\
\text { Kotschwar (2001) }\end{array}$ \\
\hline Canada - Costa Rica & 1er octobre 02 & & $\begin{array}{l}\text { Accord de libre- } \\
\text { échange }\end{array}$ & oui & $\begin{array}{l}\text { Piermartini et Budetta } \\
\text { (2006) } \\
\text { Kotschwar (2001) }\end{array}$ \\
\hline Canada - Israël & 1er janvier 97 & & $\begin{array}{l}\text { Accord de libre- } \\
\text { échange }\end{array}$ & oui & $\begin{array}{l}\text { Piermartini et Budetta } \\
\text { (2006) }\end{array}$ \\
\hline $\begin{array}{l}\text { Communauté des } \\
\text { Caraïbes (CARICOM) }\end{array}$ & 1er juillet 97 & $\begin{array}{l}\text { Antigua et Barbuda, Bahamas, } \\
\text { Barbade, Belize, Dominique, } \\
\text { Grenade, Guyana, Haïti, } \\
\text { Jamaïque, Montserrat, Sainte- } \\
\text { Lucie, Saint-Christophe et Nievès, } \\
\text { Saint-Vincent et les Grenadines, } \\
\text { Suriname, Trinidad, Tobago }\end{array}$ & Union douanière & oui & $\begin{array}{l}\text { Piermartini et Budetta } \\
(2006) \\
\text { Kotschwar (2001) }\end{array}$ \\
\hline $\begin{array}{l}\text { CARICOM- } \\
\text { République } \\
\text { dominicaine }^{*}\end{array}$ & 5 février 02 & $\begin{array}{c}\text { Suriname, Guyana, République } \\
\text { dominicaine, Barbade, Jamaïque, } \\
\text { Trinidad et Tobago }\end{array}$ & $\begin{array}{l}\text { Accord de libre- } \\
\text { échange }\end{array}$ & oui & Kotschwar (2001) \\
\hline $\begin{array}{l}\text { Accord de libre- } \\
\text { échange de } \\
\text { l'Amérique centrale- } \\
\text { République } \\
\text { dominicaine- États- } \\
\text { Unis (ALECA-RD) } \\
\end{array}$ & $1^{\text {er }}$ mars 06 & $\begin{array}{c}\text { États-Unis, Costa Rica, } \\
\text { Guatemala, El Salvador, } \\
\text { Honduras, Nicaragua, République } \\
\text { dominicaine }\end{array}$ & $\begin{array}{l}\text { Accord de libre- } \\
\text { échange }\end{array}$ & oui & $\begin{array}{l}\text { Piermartini et Budetta } \\
\text { (2006) }\end{array}$ \\
\hline $\begin{array}{l}\text { Accord de libre- } \\
\text { échange d'Europe } \\
\text { centrale (ALECE) }\end{array}$ & 1er mars 93 & $\begin{array}{l}\text { Bulgarie, République tchèque, } \\
\text { Hongrie, Pologne, Roumanie, } \\
\text { Slovaquie, Slovénie }\end{array}$ & $\begin{array}{l}\text { Accord de libre- } \\
\text { échange }\end{array}$ & oui & $\begin{array}{l}\text { Piermartini et Budetta } \\
\text { (2006) }\end{array}$ \\
\hline
\end{tabular}


TAD/TC/WP(2007)12/FINAL

\begin{tabular}{|c|c|c|c|c|c|}
\hline Accord & $\begin{array}{l}\text { Date d'entrée } \\
\text { en vigueur }\end{array}$ & membres & Type & Chapitre sur les OTC & Examinés par \\
\hline $\begin{array}{l}\text { Communauté } \\
\text { Économique et } \\
\text { Monétaire de } \\
\text { l'Afrique Centrale } \\
\text { (CEMAC) }\end{array}$ & 24 juin 99 & $\begin{array}{l}\text { Cameroun, Centrafrique Congo, } \\
\text { Gabon, Guinée équatoriale, } \\
\text { Tchad }\end{array}$ & $\begin{array}{c}\text { Accord } \\
\text { préférentiel }\end{array}$ & non & $\begin{array}{l}\text { Piermartini et Budetta } \\
(2006)\end{array}$ \\
\hline $\begin{array}{l}\text { Accord de } \\
\text { partenariat } \\
\text { économique } \\
\text { stratégique } \\
\text { transpacifique } \\
(\mathrm{P} 4)^{*}\end{array}$ & 8 novembre 06 & $\begin{array}{l}\text { Nouvelle-Zélande, Singapour, } \\
\text { Brunei Darussalam, Chili }\end{array}$ & $\begin{array}{l}\text { Accord de libre- } \\
\text { échange }\end{array}$ & oui & auteur \\
\hline Chili - Chine* & 1er octobre 06 & & $\begin{array}{l}\text { Accord de libre- } \\
\text { échange }\end{array}$ & oui & auteur \\
\hline Chili - Costa Rica & 15 février 02 & $\begin{array}{c}\text { (dans le cadre de l'accord de } \\
\text { libre-échange Chili- Amérique } \\
\text { centrale) }\end{array}$ & $\begin{array}{l}\text { Accord de libre- } \\
\text { échange }\end{array}$ & oui & $\begin{array}{l}\text { auteur } \\
\text { Kotschwar (2001) }\end{array}$ \\
\hline Chili - El Salvador & 15 février 02 & $\begin{array}{l}\text { (dans le cadre de l'accord de } \\
\text { libre-échange Chili- Amérique } \\
\text { centrale) }\end{array}$ & & oui & $\begin{array}{l}\text { auteur } \\
\text { Kotschwar (2001) }\end{array}$ \\
\hline Chili - Canada & 5 juillet 97 & & $\begin{array}{l}\text { Accord de libre- } \\
\text { échange }\end{array}$ & $\begin{array}{c}\text { pour les } \\
\text { télécommunications } \\
\text { uniquement }\end{array}$ & $\begin{array}{l}\text { auteur } \\
\text { Piermartini et Budetta } \\
\text { (2006) } \\
\text { Kotschwar (2001) }\end{array}$ \\
\hline Chili - UE & 1er février 03 & & $\begin{array}{l}\text { Accord de libre- } \\
\text { échange }\end{array}$ & oui & $\begin{array}{l}\text { auteur } \\
\text { Piermartini et Budetta } \\
\text { (2006) } \\
\text { ECDPM (à paraître) }\end{array}$ \\
\hline Chili - AELE & $\begin{array}{l}\text { 1er décembre } \\
04\end{array}$ & $\begin{array}{l}\text { Chili, Islande, Liechtenstein, } \\
\text { Norvège, Suisse }\end{array}$ & $\begin{array}{l}\text { Accord de libre- } \\
\text { échange }\end{array}$ & oui & auteur \\
\hline Chili - Mexique & 1er août 99 & & $\begin{array}{l}\text { Accord de libre- } \\
\text { échange }\end{array}$ & oui & $\begin{array}{l}\text { auteur } \\
\text { Piermartini et Budetta } \\
\text { (2006) } \\
\text { Kotschwar (2001) }\end{array}$ \\
\hline $\begin{array}{l}\text { Chili - République } \\
\text { de Corée }\end{array}$ & 1er avril 04 & & $\begin{array}{l}\text { Accord de libre- } \\
\text { échange }\end{array}$ & oui & auteur \\
\hline Chili - United States & 1er janvier 04 & & $\begin{array}{l}\text { Accord de libre- } \\
\text { échange }\end{array}$ & oui & $\begin{array}{l}\text { auteur } \\
\text { Piermartini et Budetta } \\
\text { (2006) }\end{array}$ \\
\hline $\begin{array}{l}\text { Association latino- } \\
\text { américaine } \\
\text { d'intégration } \\
\text { (ALADI) }\end{array}$ & 18 mars 81 & $\begin{array}{l}\text { Argentine, Colombie, Paraguay, } \\
\text { Bolivie, Cuba, Pérou, Brésil, } \\
\text { Equateur, Uruguay, Chili, } \\
\text { Mexique, Venezuela }\end{array}$ & $\begin{array}{c}\text { Accord } \\
\text { préférentiel }\end{array}$ & oui & $\begin{array}{l}\text { auteur } \\
\text { Piermartini et Budetta } \\
\text { (2006) }\end{array}$ \\
\hline $\begin{array}{l}\text { Chine - Hong Kong, } \\
\text { Chine }\end{array}$ & 1er janvier 04 & & $\begin{array}{l}\text { Accord de libre- } \\
\text { échange }\end{array}$ & non & $\begin{array}{l}\text { Piermartini et Budetta } \\
(2006)\end{array}$ \\
\hline $\begin{array}{l}\text { China - Macao, } \\
\text { Chine }\end{array}$ & 1er janvier 04 & & $\begin{array}{l}\text { Accord de libre- } \\
\text { échange }\end{array}$ & non & $\begin{array}{l}\text { Piermartini et Budetta } \\
\text { (2006) }\end{array}$ \\
\hline UE - Algérie & $\begin{array}{l}\text { 1er septembre } \\
05\end{array}$ & & $\begin{array}{l}\text { Accord de libre- } \\
\text { échange }\end{array}$ & oui & $\begin{array}{l}\text { Piermartini et Budetta } \\
(2006)\end{array}$ \\
\hline UE - Bulgarie & $\begin{array}{l}\text { 31décembre } \\
93\end{array}$ & & $\begin{array}{l}\text { Accord de libre- } \\
\text { échange }\end{array}$ & oui & $\begin{array}{l}\text { Piermartini et Budetta } \\
(2006)\end{array}$ \\
\hline
\end{tabular}




\begin{tabular}{|c|c|c|c|c|c|}
\hline Accord & $\begin{array}{l}\text { Date d'entrée } \\
\text { en vigueur }\end{array}$ & membres & Type & $\begin{array}{l}\text { Chapitre sur les } \\
\text { OTC }\end{array}$ & Examiné par \\
\hline UE - Croatie & 1er mars 02 & & $\begin{array}{l}\text { Accord de libre- } \\
\text { échange }\end{array}$ & non & $\begin{array}{l}\text { Piermartini et Budetta } \\
(2006)\end{array}$ \\
\hline UE - Jordanie & 1er mai 02 & & $\begin{array}{l}\text { Accord de libre- } \\
\text { échange }\end{array}$ & oui & $\begin{array}{l}\text { Piermartini et Budetta } \\
(2006)\end{array}$ \\
\hline UE - Egypte & 1er juin 04 & & $\begin{array}{l}\text { Accord de libre- } \\
\text { échange }\end{array}$ & oui & $\begin{array}{l}\text { Piermartini et Budetta } \\
(2006)\end{array}$ \\
\hline UE- Israël & 1er juin 00 & & $\begin{array}{l}\text { Accord de libre- } \\
\text { échange }\end{array}$ & non & $\begin{array}{l}\text { Piermartini et Budetta } \\
(2006)\end{array}$ \\
\hline UE- Liban & 1er mars 03 & & $\begin{array}{l}\text { Accord de libre- } \\
\text { échange }\end{array}$ & oui & $\begin{array}{l}\text { Piermartini et Budetta } \\
(2006)\end{array}$ \\
\hline $\begin{array}{l}\text { UE - Autorité } \\
\text { palestinienne }\end{array}$ & 1er juillet 97 & & $\begin{array}{l}\text { Accord de libre- } \\
\text { échange }\end{array}$ & oui & $\begin{array}{l}\text { Piermartini et Budetta } \\
(2006)\end{array}$ \\
\hline UE - Roumanie & 1er mai 93 & & $\begin{array}{l}\text { Accord de libre- } \\
\text { échange }\end{array}$ & oui & $\begin{array}{l}\text { Piermartini et Budetta } \\
(2006)\end{array}$ \\
\hline UE - Afrique du Sud & 1er janvier 00 & & $\begin{array}{l}\text { Accord de libre- } \\
\text { échange }\end{array}$ & oui & $\begin{array}{l}\text { Piermartini et Budetta } \\
\text { (2006) } \\
\text { ECDPM (à paraître) }\end{array}$ \\
\hline $\begin{array}{l}\text { UE - Suisse et } \\
\text { Liechtenstein }\end{array}$ & 1er janvier 73 & & $\begin{array}{l}\text { Accord de libre- } \\
\text { échange }\end{array}$ & oui & $\begin{array}{l}\text { Piermartini et Budetta } \\
(2006)\end{array}$ \\
\hline UE - Syrie & 1er juillet 77 & & $\begin{array}{l}\text { Accord de libre- } \\
\text { échange }\end{array}$ & non & $\begin{array}{l}\text { Piermartini et Budetta } \\
(2006)\end{array}$ \\
\hline UE - Tunisie & 1er mars 98 & & $\begin{array}{l}\text { Accord de libre- } \\
\text { échange }\end{array}$ & oui & $\begin{array}{l}\text { Piermartini et Budetta } \\
\text { (2006) } \\
\text { ECDPM (à paraître) }\end{array}$ \\
\hline UE - Turquie & 1er janvier 96 & & Union douanière & oui & $\begin{array}{l}\text { Piermartini et Budetta } \\
\text { (2006) }\end{array}$ \\
\hline UE (Traité de Rome) & 1er janvier 58 & & Union douanière & oui & $\begin{array}{l}\text { Piermartini et Budetta } \\
(2006)\end{array}$ \\
\hline $\begin{array}{l}\text { Espace économique } \\
\text { européen (EEE) }\end{array}$ & 1er janvier 94 & $\begin{array}{l}\text { UE, Islande, Liechtenstein, } \\
\text { Norvège }\end{array}$ & Union douanière & oui & $\begin{array}{l}\text { Piermartini et Budetta } \\
(2006)\end{array}$ \\
\hline $\begin{array}{l}\text { Association } \\
\text { européenne de libre- } \\
\text { échange (AELE, } \\
\text { Convention de } \\
\text { Stockholm) }\end{array}$ & 3 mai 60 & $\begin{array}{l}\text { Islande, Liechtenstein, Norvège, } \\
\text { Suisse }\end{array}$ & $\begin{array}{l}\text { Accord de libre- } \\
\text { échange }\end{array}$ & oui & $\begin{array}{l}\text { Piermartini et Budetta } \\
(2006)\end{array}$ \\
\hline AELE - Bulgarie & 1er juillet 93 & & $\begin{array}{l}\text { Accord de libre- } \\
\text { échange }\end{array}$ & oui & $\begin{array}{l}\text { Piermartini et Budetta } \\
\text { (2006) }\end{array}$ \\
\hline AELE - Mexique & 1er juillet 01 & & $\begin{array}{l}\text { Accord de libre- } \\
\text { échange }\end{array}$ & oui & $\begin{array}{l}\text { Piermartini et Budetta } \\
(2006)\end{array}$ \\
\hline $\begin{array}{l}\text { AELE- Autorité } \\
\text { palestinienne }\end{array}$ & 1er juillet 99 & & $\begin{array}{l}\text { Accord de libre- } \\
\text { échange }\end{array}$ & oui & $\begin{array}{l}\text { Piermartini et Budetta } \\
(2006)\end{array}$ \\
\hline AELE - Roumanie & 1er mai 93 & & $\begin{array}{l}\text { Accord de libre- } \\
\text { échange }\end{array}$ & oui & $\begin{array}{l}\text { Piermartini et Budetta } \\
(2006)\end{array}$ \\
\hline AELE - Turquie & 1er avril 92 & & $\begin{array}{l}\text { Accord de libre- } \\
\text { échange }\end{array}$ & oui & $\begin{array}{l}\text { Piermartini et Budetta } \\
(2006)\end{array}$ \\
\hline $\begin{array}{l}\text { Conseil de } \\
\text { coopération du Golfe } \\
\text { (CCG) }\end{array}$ & 1er janvier 03 & $\begin{array}{l}\text { Bahreïn, Koweït, Oman, Qatar, } \\
\text { Arabie Saoudite, EAU }\end{array}$ & Union douanière & non & $\begin{array}{l}\text { Piermartini et Budetta } \\
\text { (2006) }\end{array}$ \\
\hline AELE - Israël & 1er janvier 93 & & $\begin{array}{l}\text { Accord de libre- } \\
\text { échange }\end{array}$ & oui & $\begin{array}{l}\text { Piermartini et Budetta } \\
(2006)\end{array}$ \\
\hline
\end{tabular}


TAD/TC/WP(2007)12/FINAL

\begin{tabular}{|c|c|c|c|c|c|}
\hline Accord & $\begin{array}{l}\text { Date d'entrée } \\
\text { en vigueur }\end{array}$ & membres & Type & $\begin{array}{l}\text { Chapitre sur les } \\
\text { OTC }\end{array}$ & Examiné par \\
\hline Groupe des Trois* & 1er janvier -95 & Colombie, Mexique, Venezuela & $\begin{array}{l}\text { Accord de libre- } \\
\text { échange }\end{array}$ & oui & $\begin{array}{l}\text { Piermartini et Budetta } \\
\text { (2006) } \\
\text { Kotschwar (2001) }\end{array}$ \\
\hline Mexique -Bolivie & 1er janvier 95 & & $\begin{array}{l}\text { Accord de libre- } \\
\text { échange }\end{array}$ & oui & Kotschwar (2001) \\
\hline $\begin{array}{l}\text { Mexique - Costa } \\
\text { Rica }\end{array}$ & 1er janvier 95 & & $\begin{array}{l}\text { Accord de libre- } \\
\text { échange }\end{array}$ & oui & Kotschwar (2001) \\
\hline Mexique-UE & 1er juillet 00 & & $\begin{array}{l}\text { Accord de libre- } \\
\text { échange }\end{array}$ & oui & $\begin{array}{l}\text { Piermartini et Budetta } \\
\text { (2006) } \\
\text { ECDPM (à paraître) }\end{array}$ \\
\hline Mexique - Israël & 1er juillet -00 & & $\begin{array}{l}\text { Accord de libre- } \\
\text { échange }\end{array}$ & non & $\begin{array}{l}\text { Piermartini et Budetta } \\
\text { (2006) }\end{array}$ \\
\hline Mexique - Japon & 1er avril 05 & & $\begin{array}{l}\text { Accord de libre- } \\
\text { échange }\end{array}$ & oui & $\begin{array}{l}\text { Piermartini et Budetta } \\
\text { (2006) }\end{array}$ \\
\hline Mexique- Nicaragua & 1er juillet 98 & & $\begin{array}{l}\text { Accord de libre- } \\
\text { échange }\end{array}$ & oui & $\begin{array}{l}\text { Piermartini et Budetta } \\
\text { (2006) } \\
\text { Kotschwar (2001) }\end{array}$ \\
\hline $\begin{array}{l}\text { Mexique -Triangle } \\
\text { du Nord }\end{array}$ & 14 mars 01 & $\begin{array}{l}\text { El Salvador, Guatemala, } \\
\text { Honduras, Mexique }\end{array}$ & $\begin{array}{l}\text { Accord de libre- } \\
\text { échange }\end{array}$ & oui & $\begin{array}{l}\text { Piermartini et Budetta } \\
(2006) \\
\text { Kotschwar (2001) }\end{array}$ \\
\hline Mexique -Uruguay & 15 juillet 04 & & $\begin{array}{l}\text { Accord de libre- } \\
\text { échange }\end{array}$ & oui & $\begin{array}{l}\text { Piermartini et Budetta } \\
\text { (2006) }\end{array}$ \\
\hline ALENA & 1er janvier 94 & États-Unis, Mexique, Canada & $\begin{array}{l}\text { Accord de libre- } \\
\text { échange }\end{array}$ & oui & $\begin{array}{l}\text { Piermartini et Budetta } \\
(2006) \\
\text { Kotschwar }\end{array}$ \\
\hline MERCOSUR & 29 novembre 91 & $\begin{array}{c}\text { Argentine, Brésil, Paraguay, } \\
\text { Uruguay }\end{array}$ & Union douanière & oui & $\begin{array}{l}\text { Piermartini et Budetta } \\
(2006) \\
\text { Kotschwar (2001) }\end{array}$ \\
\hline Maroc - UE & 1er mars 00 & & $\begin{array}{l}\text { Accord de libre- } \\
\text { échange }\end{array}$ & oui & $\begin{array}{l}\text { auteur } \\
\text { Piermartini et Budetta } \\
\text { (2006) }\end{array}$ \\
\hline Maroc - AELE & 1er décembre 99 & & $\begin{array}{l}\text { Accord de libre- } \\
\text { échange }\end{array}$ & oui & $\begin{array}{l}\text { auteur } \\
\text { Piermartini et Budetta } \\
\text { (2006) }\end{array}$ \\
\hline Maroc -Turquie & 1er janvier 06 & & $\begin{array}{l}\text { Accord de libre- } \\
\text { échange }\end{array}$ & oui & auteur \\
\hline Maroc - États-Unis & 1er janvier 06 & & $\begin{array}{l}\text { Accord de libre- } \\
\text { échange }\end{array}$ & oui & $\begin{array}{l}\text { auteur } \\
\text { Piermartini et Budetta } \\
\text { (2006) }\end{array}$ \\
\hline Accord d'Agadir * & 27 mars 07 & Maroc, Tunisie, Egypte, Jordanie & $\begin{array}{l}\text { Accord de libre- } \\
\text { échange }\end{array}$ & oui & auteur \\
\hline Singapour -Corée* & 2 mars 06 & & $\begin{array}{l}\text { Accord de libre- } \\
\text { échange }\end{array}$ & oui & auteur \\
\hline $\begin{array}{l}\text { Singapour - } \\
\text { Panama* }^{*}\end{array}$ & 24 juillet 06 & & $\begin{array}{l}\text { Accord de libre- } \\
\text { échange }\end{array}$ & oui & auteur \\
\hline & & & & & \\
\hline & & & & & \\
\hline
\end{tabular}




\begin{tabular}{|c|c|c|c|c|c|}
\hline Accord & $\begin{array}{l}\text { Date d'entrée } \\
\text { en vigueur }\end{array}$ & membres & Type & $\begin{array}{c}\text { Chapitre sur les } \\
\text { OTC }\end{array}$ & Examinés par \\
\hline $\begin{array}{l}\text { Singapour - États- } \\
\text { Unis }\end{array}$ & 1er janvier 04 & & $\begin{array}{l}\text { Accord de libre- } \\
\text { échange }\end{array}$ & oui & $\begin{array}{l}\text { auteur } \\
\text { Piermartini et Budetta } \\
\text { (2006) }\end{array}$ \\
\hline $\begin{array}{l}\text { Singapour - } \\
\text { Australie (SAFTA) }\end{array}$ & 28 juillet 03 & & $\begin{array}{l}\text { Accord de libre- } \\
\text { échange }\end{array}$ & oui & $\begin{array}{l}\text { auteur } \\
\text { Piermartini et Budetta } \\
\text { (2006) }\end{array}$ \\
\hline $\begin{array}{l}\text { Singapour - } \\
\text { Nouvelle- Zélande }\end{array}$ & 1er janvier 01 & & $\begin{array}{l}\text { Accord de libre- } \\
\text { échange }\end{array}$ & oui & $\begin{array}{l}\text { auteur } \\
\text { Piermartini et Budetta } \\
\text { (2006) }\end{array}$ \\
\hline Singapour - Japon & 30 novembre 02 & & $\begin{array}{l}\text { Accord de libre- } \\
\text { échange }\end{array}$ & oui & $\begin{array}{l}\text { auteur } \\
\text { Piermartini et Budetta } \\
\text { (2006) }\end{array}$ \\
\hline $\begin{array}{l}\text { Singapour - } \\
\text { Jordanie }\end{array}$ & 22 août 05 & & $\begin{array}{l}\text { Accord de libre- } \\
\text { échange }\end{array}$ & non & auteur \\
\hline Singapour-AELE & 1er janvier 03 & & $\begin{array}{l}\text { Accord de libre- } \\
\text { échange }\end{array}$ & oui & $\begin{array}{l}\text { auteur } \\
\text { Piermartini et Budetta } \\
\text { (2006) }\end{array}$ \\
\hline Singapour -Inde* & 1er août 2005 & & $\begin{array}{l}\text { Accord de libre- } \\
\text { échange }\end{array}$ & oui & auteur \\
\hline $\begin{array}{l}\text { Communauté de } \\
\text { développement de } \\
\text { l'Afrique australe } \\
\text { (CDAA) }\end{array}$ & $\begin{array}{l}\text { 1er septembre } \\
00\end{array}$ & $\begin{array}{c}\text { Angola, Botswana, République } \\
\text { démocratique du Congo, Lesotho, } \\
\text { Madagascar, Malawi, Île Maurice, } \\
\text { Mozambique, Namibie, Afrique du } \\
\text { Sud, Swaziland, République-Unie } \\
\text { de Tanzanie, Zambie et } \\
\text { Zimbabwe. }\end{array}$ & $\begin{array}{l}\text { Accord de libre- } \\
\text { échange }\end{array}$ & oui & $\begin{array}{l}\text { Piermartini et Budetta } \\
\text { (2006) }\end{array}$ \\
\hline $\begin{array}{l}\text { Accord d'échanges } \\
\text { préférentiels du } \\
\text { SAARC de } \\
\text { l'Association pour } \\
\text { la coopération } \\
\text { régionale de l'Asie } \\
\text { du Sud (SAPTA) }\end{array}$ & 7 décembre 95 & $\begin{array}{l}\text { Bangladesh, Bhoutan, Inde, } \\
\text { Maldives, Népal, Pakistan, Sri } \\
\text { Lanka }\end{array}$ & Accord préférentiel & oui & $\begin{array}{l}\text { Piermartini et Budetta } \\
\text { (2006) }\end{array}$ \\
\hline $\begin{array}{l}\text { Accord de } \\
\text { coopération } \\
\text { économique et } \\
\text { commerciale } \\
\text { régionale du } \\
\text { Pacifique Sud } \\
\text { (SPARTECA) }\end{array}$ & 1er janvier 81 & $\begin{array}{l}\text { Australie, Îles Cook, Fidji, Kiribati, } \\
\text { Nauru, Niue, Nouvelle-Zélande, } \\
\text { Papouasie-Nouvelle-Guinée, Îles } \\
\text { Salomon, Tonga, Tuvalu, Samoa }\end{array}$ & Accord préférentiel & non & $\begin{array}{l}\text { Piermartini et Budetta } \\
\text { (2006) }\end{array}$ \\
\hline Turquie - Israël & 1er mai 97 & & $\begin{array}{l}\text { Accord de libre- } \\
\text { échange }\end{array}$ & oui & $\begin{array}{l}\text { Piermartini et Budetta } \\
\text { (2006) }\end{array}$ \\
\hline $\begin{array}{l}\text { États-Unis - } \\
\text { Jordanie }\end{array}$ & 17décembre 01 & & $\begin{array}{l}\text { Accord de libre- } \\
\text { échange }\end{array}$ & non & $\begin{array}{l}\text { Piermartini et Budetta } \\
\text { (2006) }\end{array}$ \\
\hline $\begin{array}{l}\text { United States - } \\
\text { Israël }\end{array}$ & 19 août 85 & & $\begin{array}{l}\text { Accord de libre- } \\
\text { échange }\end{array}$ & non & $\begin{array}{l}\text { Piermartini et Budetta } \\
(2006)\end{array}$ \\
\hline $\begin{array}{l}\text { Union Economique } \\
\text { et Monétaire Ouest } \\
\text { Africaine (UEMOA) }\end{array}$ & 1 er janvier 00 & $\begin{array}{l}\text { Bénin, Burkina Faso, Côte d'Ivoire, } \\
\text { Guinée Bissau, Mali, Niger, } \\
\text { Sénégal, Togo }\end{array}$ & Accord préférentiel & non & $\begin{array}{l}\text { Piermartini et Budetta } \\
\text { (2006) }\end{array}$ \\
\hline
\end{tabular}

Source : OMC - Accords commerciaux régionaux - Portail et CIB Database.

Note : * non notifié à l'OMC. Les accords apparaissant en surbrillance ont été examinés par l'auteur. 
Informations supplémentaires au sujet de l'échantillon

ACR examinés, par types de parties

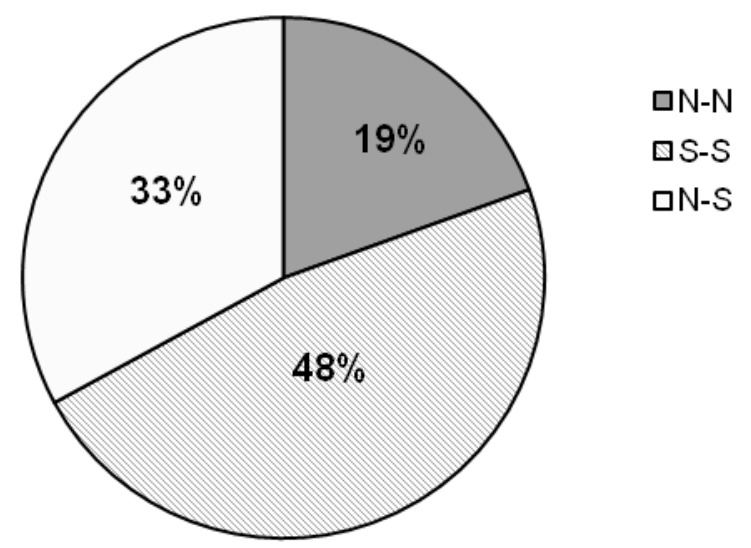

Source: OMC (2005). La catégorie "N" ou Nord renvoie à tous les pays à revenu élevé (d'après le classement de la Banque
ACR examinés, par types d'accords

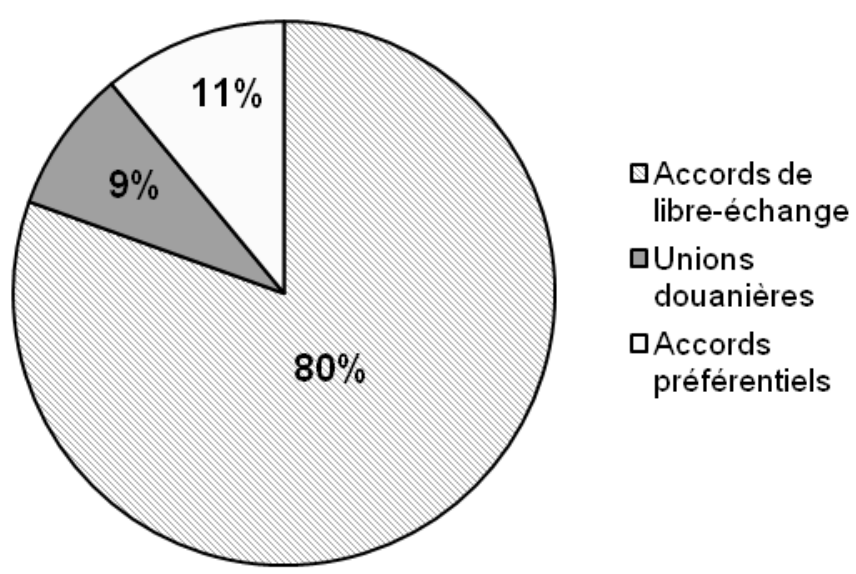

Source: Portail OMC sur les accords commerciaux régionaux

\section{ACR examinés, par date d'entrée en vigueur}

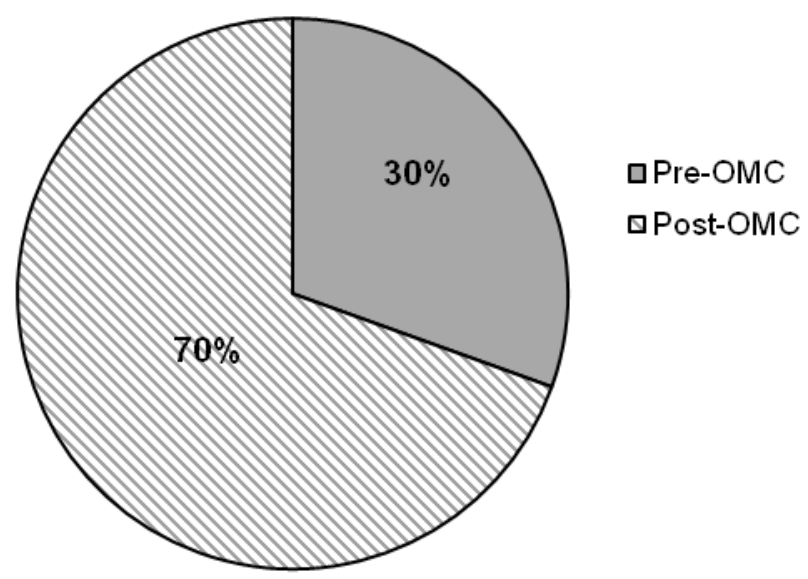

Source: Portail OMC sur les accords commerciaux 
TAD/TC/WP(2007)12/FINAL

ANNEXE II. INFORMATIONS DE RÉFÉRENCE CONCERNANT LES ÉTUDES DE CAS

Tableau 7. Tour d'horizon des dispositions juridiques contenues dans les ACR conclus par le Chili

\begin{tabular}{|c|c|c|c|c|c|c|c|c|c|c|c|c|}
\hline Catégories & Questions & $\begin{array}{l}\text { Chili- } \\
\text { Canada }\end{array}$ & $\begin{array}{c}\text { Chili- } \\
\text { Mexique }\end{array}$ & $\begin{array}{l}\text { Chili-Costa } \\
\text { Rica }\end{array}$ & $\begin{array}{l}\text { Chili-El } \\
\text { Salvador }\end{array}$ & Chili-UE & $\begin{array}{l}\text { Chili-États- } \\
\text { Unis }\end{array}$ & Chili-Corée & $\begin{array}{l}\text { Chili- } \\
\text { AELE }\end{array}$ & $\begin{array}{l}\text { Chili- } \\
\text { Chine }\end{array}$ & $\begin{array}{c}\text { Accord } \\
\text { transpacifique }\end{array}$ & TOTAL \\
\hline \multirow[t]{2}{*}{$\begin{array}{l}\text { 1. Référence à } \\
\text { l'Accord sur les OTC } \\
\text { de l'OMC }\end{array}$} & $\begin{array}{l}\text { L'accord fait-il référence à } \\
\text { l'Accord de l'OMC (ses } \\
\text { objectifs, règles et/ou } \\
\text { dispositions)? }\end{array}$ & oui & oui & oui & oui & oui & oui & oui & oui & oui & oui & 10 sur 10 \\
\hline & $\begin{array}{l}\text { Le chapitre sur les OTC de } \\
\text { l'accord vise-t-il des } \\
\text { objectifs d'une plus grande } \\
\text { portée? }\end{array}$ & non & non & non & non & $\begin{array}{c}\text { oui, } \\
\text { renforcement } \\
\text { des échanges } \\
\text { et de } \\
\text { l'investissement }\end{array}$ & $\begin{array}{l}\text { oui, meilleure } \\
\text { facilitation des } \\
\text { échanges }\end{array}$ & non & non & non & $\begin{array}{l}\text { oui, meilleure } \\
\text { facilitation des } \\
\text { échanges }\end{array}$ & 3 sur 10 \\
\hline \multirow[t]{3}{*}{ 2. Harmonisation } & $\begin{array}{l}\text { L'accord impose-t-il aux } \\
\text { parties ou les encourage-t- } \\
\text { il à harmoniser leurs } \\
\text { règlements techniques, } \\
\text { normes et procédures } \\
\text { d'évaluation de la } \\
\text { conformité ? }\end{array}$ & non & compatibilité & compatibilité & compatibilité & oui & $\begin{array}{l}\text { oui (les } \\
\text { encourage } \\
\text { seulement) }\end{array}$ & oui & non & oui & oui & 5 sur 10 \\
\hline & $\begin{array}{l}\text { Encourage-t-il l'utilisation } \\
\text { des normes et guides } \\
\text { internationaux? }\end{array}$ & non & oui & oui & oui & oui & oui & oui & non & oui & oui & 8 sur 10 \\
\hline & $\begin{array}{l}\text { Encourage-t-il l'utilisation } \\
\text { des normes et guides } \\
\text { régionaux? }\end{array}$ & non & non & non & non & non & non & non & non & non & non & 0 sur 10 \\
\hline $\begin{array}{l}\text { 3. Acceptation des } \\
\text { règlements } \\
\text { techniques comme } \\
\text { équivalents }\end{array}$ & $\begin{array}{l}\text { L'accord impose-t-il aux } \\
\text { parties ou les encourage-t- } \\
\text { il à accepter comme } \\
\text { équivalents les règlements } \\
\text { techniques et les normes } \\
\text { des autres parties? }\end{array}$ & non & compatibilité & compatibilité & compatibilité & $\begin{array}{l}\text { oui (les } \\
\text { encourage } \\
\text { seulement) }\end{array}$ & $\begin{array}{l}\text { oui (les } \\
\text { encourage } \\
\text { seulement } \\
\text { pour les } \\
\text { règlements } \\
\text { techniques) }\end{array}$ & compatibilité & non & $\begin{array}{l}\text { oui (les } \\
\text { encourage } \\
\text { seulement } \\
\text { pour les } \\
\text { règlements } \\
\text { techniques) }\end{array}$ & $\begin{array}{l}\text { oui (les } \\
\text { encourage } \\
\text { seulement } \\
\text { pour les } \\
\text { règlements } \\
\text { techniques) }\end{array}$ & 4 sur 10 \\
\hline
\end{tabular}


TAD/TC/WP(2007)12/FINAL

\begin{tabular}{|c|c|c|c|c|c|c|c|c|c|c|c|c|}
\hline Catégories & Questions & $\begin{array}{l}\text { Chili- } \\
\text { Canada }\end{array}$ & $\begin{array}{l}\text { Chili- } \\
\text { Mexique }\end{array}$ & $\begin{array}{l}\text { Chili- Costa } \\
\text { Rica }\end{array}$ & $\begin{array}{l}\text { Chili-EI } \\
\text { Salvador }\end{array}$ & Chili-UE & $\begin{array}{l}\text { Chili-États- } \\
\text { Unis }\end{array}$ & Chili- Corée & $\begin{array}{l}\text { Chili- } \\
\text { AELE }\end{array}$ & $\begin{array}{l}\text { Chili- } \\
\text { Chine }\end{array}$ & $\begin{array}{c}\text { Accord } \\
\text { transpacifique }\end{array}$ & TOTAL \\
\hline $\begin{array}{l}\text { 3. Acceptation des } \\
\text { règlements } \\
\text { techniques comme } \\
\text { équivalents (suite) }\end{array}$ & $\begin{array}{l}\text { Leur impose-t-il d'expliquer } \\
\text { les raisons pour lesquelles } \\
\text { elles n'acceptent pas } \\
\text { l'équivalence des } \\
\text { règlements techniques des } \\
\text { autres parties? }\end{array}$ & non & oui & oui & oui & non & oui & oui & non & oui & oui & 7 sur 10 \\
\hline \multirow[t]{5}{*}{$\begin{array}{l}\text { 4. Reconnaissance } \\
\text { (mutuelle) des } \\
\text { évaluations de la } \\
\text { conformité }\end{array}$} & $\begin{array}{l}\text { L'accord préconise-t-il une } \\
\text { reconnaissance (mutuelle) } \\
\text { des règlements techniques } \\
\text { et des normes? }\end{array}$ & non & non & non & non & non & non & non & non & non & non & 0 sur 10 \\
\hline & $\begin{array}{l}\text { L'accord préconise-t-il une } \\
\text { reconnaissance (mutuelle) } \\
\text { des résultats des } \\
\text { évaluations de la } \\
\text { conformité ? }\end{array}$ & oui & oui & oui & oui & $\begin{array}{l}\text { oui, par des } \\
\text { mécanismes } \\
\text { très variés }\end{array}$ & $\begin{array}{l}\text { oui, par des } \\
\text { mécanismes } \\
\text { très variés }\end{array}$ & oui & non & oui & oui & 9 sur 10 \\
\hline & $\begin{array}{l}\text { Impose-t-il aux parties } \\
\text { d'expliquer leurs raisons en } \\
\text { cas de non- } \\
\text { reconnaissance? }\end{array}$ & non & non & oui & oui & non & oui & oui & non & oui & oui & 6 sur 10 \\
\hline & $\begin{array}{l}\text { S'accompagne-t-il d'un } \\
\text { accord de reconnaissance } \\
\text { mutuelle (ARM) distinct? }\end{array}$ & non & non & non & non & oui & non & non & non & non & non & 1 sur 10 \\
\hline & $\begin{array}{l}\text { Encourage-t-il la conclusion } \\
\text { d'un ARM distinct? }\end{array}$ & non & oui & oui & oui & oui & oui & non & non & oui & oui & 7 sur 10 \\
\hline 5. Transparence & $\begin{array}{l}\text { L'accord contient-il des } \\
\text { dispositions sur la } \\
\text { transparence? }\end{array}$ & non & oui & oui & oui & oui & oui & oui & non & oui & oui & 8 sur 10 \\
\hline
\end{tabular}


TAD/TC/WP(2007)12/FINAL

\begin{tabular}{|c|c|c|c|c|c|c|c|c|c|c|c|c|}
\hline Catégories & Questions & $\begin{array}{l}\text { Chili- } \\
\text { Canada }\end{array}$ & Chili- Mexique & $\begin{array}{l}\text { Chili-Costa } \\
\text { Rica }\end{array}$ & $\begin{array}{l}\text { Chili-El } \\
\text { Salvador }\end{array}$ & Chili- UE & $\begin{array}{l}\text { Chili-États- } \\
\text { Unis }\end{array}$ & Chili-Corée & $\begin{array}{l}\text { Chili- } \\
\text { AELE }\end{array}$ & $\begin{array}{l}\text { Chili- } \\
\text { Chine }\end{array}$ & $\begin{array}{c}\text { Accord } \\
\text { transpacifique }\end{array}$ & TOTAL \\
\hline \multirow[t]{4}{*}{$\begin{array}{l}\text { 5. Transparence } \\
\text { (suite) }\end{array}$} & $\begin{array}{l}\text { Impose-t-il aux membres } \\
\text { de procéder à des } \\
\text { consultations et de } \\
\text { notifier les règlements et } \\
\text { procédures au niveau } \\
\text { bilatéral ou régional } \\
\text { avant leur adoption? }\end{array}$ & non & $\begin{array}{l}\text { oui, et chaque } \\
\text { partie doit } \\
\text { autoriser des } \\
\text { représentants } \\
\text { de l'autre partie } \\
\text { à participer à } \\
\text { l'élaboration de } \\
\text { mesures } \\
\text { fondées sur les } \\
\text { normes }\end{array}$ & oui & oui & oui & $\begin{array}{c}\text { oui, et chaque } \\
\text { partie doit } \\
\text { autoriser des } \\
\text { représentants } \\
\text { de l'autre partie } \\
\text { à participer à } \\
\text { l'élaboration de } \\
\text { mesures } \\
\text { relatives aux } \\
\text { normes }\end{array}$ & $\begin{array}{l}\text { oui, et chaque } \\
\text { partie doit } \\
\text { autoriser des } \\
\text { représentants } \\
\text { de l'autre partie } \\
\text { à participer à } \\
\text { l'élaboration de } \\
\text { mesures } \\
\text { relatives aux } \\
\text { normes }\end{array}$ & non & oui & oui & 8 sur 10 \\
\hline & $\begin{array}{l}\text { Fixe-t-il un délai de } \\
\text { réception des } \\
\text { observations des autres } \\
\text { parties? }\end{array}$ & non & oui & oui & oui & non & oui & oui & non & oui & oui & 7 sur 10 \\
\hline & $\begin{array}{l}\text { Ce délai est-il } \\
\text { expressément supérieur } \\
\text { à } 60 \text { jours? }\end{array}$ & non & $\begin{array}{c}\text { non, « } 60 \text { jours } \\
\text { au moins » }\end{array}$ & $\begin{array}{c}\text { non, « } 60 \text { jours } \\
\text { au moins » }\end{array}$ & $\begin{array}{c}\text { non, « } 60 \text { jours } \\
\text { au moins » }\end{array}$ & non & $\begin{array}{c}\text { non, « } 60 \text { jours } \\
\text { au moins » }\end{array}$ & $\begin{array}{c}\text { non, « } 60 \text { jours } \\
\text { au moins » }\end{array}$ & non & $\begin{array}{l}\text { non, « } 60 \\
\text { jours au } \\
\text { moins » }\end{array}$ & $\begin{array}{c}\text { non, « } 60 \text { jours } \\
\text { au moins » }\end{array}$ & 0 sur 10 \\
\hline & $\begin{array}{l}\text { Impose-t-il la mise en } \\
\text { place d'un point (distinct) } \\
\text { d'information? }\end{array}$ & non & non & non & non & non & non & non & non & non & non & 0 sur 10 \\
\hline $\begin{array}{l}\text { 6. Mise en œuvre et } \\
\text { règlement des } \\
\text { différends }\end{array}$ & $\begin{array}{l}\text { L'accord préconise-t-il la } \\
\text { mise en place d'un } \\
\text { organisme régional (par } \\
\text { exemple un comité) sur } \\
\text { les OTC pour suivre et } \\
\text { examiner les } \\
\text { engagements pris en } \\
\text { matière d'OTC et le } \\
\text { processus qui s'y } \\
\text { rapporte et les invite-t-il } \\
\text { à y participer? }\end{array}$ & oui & oui & oui & oui & oui & oui & oui & oui & oui & oui & 10 sur 10 \\
\hline
\end{tabular}


TAD/TC/WP(2007)12/FINAL

\begin{tabular}{|c|c|c|c|c|c|c|c|c|c|c|c|c|}
\hline Catégories & Questions & $\begin{array}{l}\text { Chili- } \\
\text { Canada }\end{array}$ & Chili-Mexique & $\begin{array}{l}\text { Chili-Costa } \\
\text { Rica }\end{array}$ & $\begin{array}{l}\text { Chili-El } \\
\text { Salvador }\end{array}$ & Chile- UE & $\begin{array}{l}\text { Chile-États- } \\
\text { Unis }\end{array}$ & Chile-Corée & $\begin{array}{l}\text { Chile- } \\
\text { AELE }\end{array}$ & $\begin{array}{l}\text { Chile- } \\
\text { Chine }\end{array}$ & $\begin{array}{c}\text { Accord } \\
\text { transpacifique }\end{array}$ & TOTAL \\
\hline \multirow[t]{3}{*}{$\begin{array}{l}\text { 6. Mise en œuvre et } \\
\text { règlement des } \\
\text { différends (suite) }\end{array}$} & $\begin{array}{l}\text { Contient-il des } \\
\text { dispositions relatives au } \\
\text { règlement des différends } \\
\text { à l'échelon régional en } \\
\text { lien avec les OTC? }\end{array}$ & non & oui & oui & oui & oui & oui & oui & non & oui & oui & 8 sur 10 \\
\hline & $\begin{array}{l}\text { Prévoit-il la tenue de } \\
\text { consultations et la } \\
\text { formulation de } \\
\text { recommandations? }\end{array}$ & non & oui & oui & oui & oui & oui & non & non & oui & oui & 7 sur 10 \\
\hline & $\begin{array}{l}\text { Prévoit-il un mécanisme } \\
\text { plus formel de règlement } \\
\text { des différents à l'échelon } \\
\text { régional? }\end{array}$ & non & non & non & non & oui & non & non & non & non & oui & 2 sur 10 \\
\hline \multirow[t]{2}{*}{$\begin{array}{l}\text { 7. Renforcement de la } \\
\text { coopération }\end{array}$} & $\begin{array}{l}\text { Contient-il des } \\
\text { dispositions spécifiques } \\
\text { relatives à l'assistance } \\
\text { technique? }\end{array}$ & non & oui & oui & oui & oui & oui & oui & non & oui & non & 7 sur 10 \\
\hline & $\begin{array}{l}\text { Prévoit-il une } \\
\text { coopération en matière } \\
\text { de métrologie? }\end{array}$ & non & oui & $\begin{array}{l}\text { oui, fondée sur } \\
\text { des mesures } \\
\text { internationales }\end{array}$ & $\begin{array}{l}\text { oui, fondée sur } \\
\text { des mesures } \\
\text { internationales }\end{array}$ & oui & non & non & non & non & non & 4 sur 10 \\
\hline
\end{tabular}


Tableau 8. Tour d'horizon des dispositions juridiques contenues dans les ACR conclus par Singapour

\begin{tabular}{|c|c|c|c|c|c|c|c|c|c|c|c|c|}
\hline Catégories & Questions & ASEAN & $\begin{array}{l}\text { Singapour- } \\
\text { Nouvelle- } \\
\text { Zélande }\end{array}$ & $\begin{array}{l}\text { Singapour- } \\
\text { Japon }\end{array}$ & $\begin{array}{l}\text { Singapour- } \\
\text { AELE }\end{array}$ & $\begin{array}{l}\text { Singapour- } \\
\text { Australie }\end{array}$ & $\begin{array}{l}\text { Singapour- } \\
\text { États-Unis }\end{array}$ & $\begin{array}{l}\text { Singapour- } \\
\text { Corée }\end{array}$ & $\begin{array}{l}\text { Singapour- } \\
\text { Panama }\end{array}$ & $\begin{array}{c}\text { Accord } \\
\text { transpacifique }\end{array}$ & $\begin{array}{l}\text { Singapour- } \\
\text { Inde }\end{array}$ & TOTAL \\
\hline \multirow[t]{2}{*}{$\begin{array}{l}\text { 1. Référence à } \\
\text { l'Accord sur les } \\
\text { OTC de l'OMC }\end{array}$} & $\begin{array}{l}\text { L'accord fait-il } \\
\text { référence à } \\
\text { l'Accord de l'OMC } \\
\text { (ses objectifs, } \\
\text { règles ettou } \\
\text { dispositions)? }\end{array}$ & oui & oui & oui & oui & oui & oui & oui & oui & oui & oui & $\begin{array}{c}10 \text { sur } \\
10\end{array}$ \\
\hline & $\begin{array}{l}\text { Le chapitre sur les } \\
\text { OTC de l'accord } \\
\text { vise-t-il des } \\
\text { objectifs d'une } \\
\text { plus grande } \\
\text { portée? }\end{array}$ & $\begin{array}{l}\text { oui, meilleure } \\
\text { intégration } \\
\text { économique }\end{array}$ & non & non & non & $\begin{array}{l}\text { oui, faciliter les } \\
\text { échanges et } \\
\text { l'investissement }\end{array}$ & non & $\begin{array}{l}\text { oui, améliorer } \\
\text { les conditions } \\
\text { pour les } \\
\text { entreprises }\end{array}$ & $\begin{array}{c}\text { oui, meilleure } \\
\text { coopération } \\
\text { réglementaire }\end{array}$ & $\begin{array}{l}\text { oui, meilleure } \\
\text { coopération } \\
\text { réglementaire }\end{array}$ & non & 5 sur 10 \\
\hline \multirow[t]{2}{*}{ 2. Harmonisation } & $\begin{array}{l}\text { L'accord impose-t- } \\
\text { il aux parties ou } \\
\text { les encourage-t-il } \\
\text { à harmoniser leurs } \\
\text { règlements } \\
\text { techniques, } \\
\text { normes et } \\
\text { procédures } \\
\text { d'évaluation de la } \\
\text { conformité? }\end{array}$ & oui & oui & non & non & oui & non & oui & oui & oui & oui & 7 sur 10 \\
\hline & $\begin{array}{l}\text { Encourage-t-il } \\
\text { l'utilisation des } \\
\text { normes et guides } \\
\text { internationaux? }\end{array}$ & oui & oui & non & oui & oui & oui & oui & oui & oui & oui & 9 sur 10 \\
\hline
\end{tabular}


TAD/TC/WP(2007)12/FINAL

\begin{tabular}{|c|c|c|c|c|c|c|c|c|c|c|c|c|}
\hline Catégories & Questions & ASEAN & $\begin{array}{l}\text { Singapour- } \\
\text { Nouvelle- } \\
\text { Zélande }\end{array}$ & $\begin{array}{c}\text { Singapour- } \\
\text { Japon }\end{array}$ & $\begin{array}{l}\text { Singapour- } \\
\text { AELE }\end{array}$ & $\begin{array}{l}\text { Singapour- } \\
\text { Australie }\end{array}$ & $\begin{array}{l}\text { Singapour- } \\
\text { États-Unis }\end{array}$ & $\begin{array}{l}\text { Singapour- } \\
\text { Corée }\end{array}$ & $\begin{array}{l}\text { Singapour- } \\
\text { Panama }\end{array}$ & $\begin{array}{c}\text { Accord } \\
\text { transpacifique }\end{array}$ & $\begin{array}{l}\text { Singapour- } \\
\text { Inde }\end{array}$ & TOTAL \\
\hline $\begin{array}{l}\text { 2. Harmonisation } \\
\text { (suite) }\end{array}$ & $\begin{array}{l}\text { Encourage-t-il } \\
\text { l'utilisation des } \\
\text { normes et guides } \\
\text { régionaux? }\end{array}$ & $\begin{array}{c}\text { oui } \\
\text { (ASEAN) }\end{array}$ & non & non & non & oui (APEC) & non & non & non & oui (APEC) & non & 3 sur 10 \\
\hline \multirow[t]{2}{*}{$\begin{array}{l}\text { 3. Acceptation des } \\
\text { règlements } \\
\text { techniques comme } \\
\text { équivalents }\end{array}$} & $\begin{array}{l}\text { L'accord impose-t-il } \\
\text { aux parties ou les } \\
\text { encourage-t-il à } \\
\text { accepter comme } \\
\text { équivalents les } \\
\text { règlements } \\
\text { techniques et les } \\
\text { normes des autres } \\
\text { parties? }\end{array}$ & non & $\begin{array}{c}\text { oui, (les } \\
\text { encourage } \\
\text { seulement) }\end{array}$ & non & non & non & non & non & $\begin{array}{l}\text { oui, (les } \\
\text { encourage } \\
\text { seulement) }\end{array}$ & $\begin{array}{l}\text { oui, (les } \\
\text { encourage } \\
\text { seulement) }\end{array}$ & $\begin{array}{c}\text { oui, (les } \\
\text { encourage } \\
\text { seulement) }\end{array}$ & 4 sur 10 \\
\hline & $\begin{array}{l}\text { Leur impose-t-il } \\
\text { d'expliquer les } \\
\text { raisons pour } \\
\text { lesquelles elles } \\
\text { n'acceptent pas } \\
\text { l'équivalence des } \\
\text { règlements } \\
\text { techniques des } \\
\text { autres parties? }\end{array}$ & non & oui & non & non & non & non & non & oui & oui & non & 3 sur 10 \\
\hline \multirow[t]{2}{*}{$\begin{array}{l}\text { 4. Reconnaissance } \\
\text { (mutuelle) des } \\
\text { évaluations de la } \\
\text { conformité }\end{array}$} & $\begin{array}{l}\text { L'accord préconise- } \\
\text { t-il une } \\
\text { reconnaissance } \\
\text { (mutuelle) des } \\
\text { règlements } \\
\text { techniques et des } \\
\text { normes? }\end{array}$ & non & non & non & non & non & non & non & non & non & non & 0 sur 10 \\
\hline & $\begin{array}{l}\text { L'accord préconise- } \\
\text { t-il une } \\
\text { reconnaissance } \\
\text { (mutuelle) des } \\
\text { résultats des } \\
\text { évaluations de la } \\
\text { conformité ? }\end{array}$ & oui & oui & oui & oui & oui & $\begin{array}{c}\text { oui (applique } \\
\text { l'ARM TEL de } \\
\text { I'APEC) }\end{array}$ & $\begin{array}{c}\text { oui, par des } \\
\text { mécanismes } \\
\text { très variés } \\
\text { (dont l'ARM } \\
\text { TEL de } \\
\text { I'APEC) }\end{array}$ & $\begin{array}{c}\text { oui, par des } \\
\text { mécanismes } \\
\text { très variés }\end{array}$ & $\begin{array}{c}\text { oui, par des } \\
\text { mécanismes très } \\
\text { variés }\end{array}$ & oui & $\begin{array}{c}10 \text { sur } \\
10\end{array}$ \\
\hline
\end{tabular}


TAD/TC/WP(2007)12/FINAL

\begin{tabular}{|c|c|c|c|c|c|c|c|c|c|c|c|c|}
\hline Catégories & Questions & ASEAN & $\begin{array}{l}\text { Singapour- } \\
\text { Nouvelle- } \\
\text { Zélande }\end{array}$ & $\begin{array}{l}\text { Singapour- } \\
\text { Japon }\end{array}$ & $\begin{array}{l}\text { Singapour- } \\
\text { AELE }\end{array}$ & $\begin{array}{l}\text { Singapour- } \\
\text { Australie }\end{array}$ & $\begin{array}{c}\text { Singapour-États- } \\
\text { Unis }\end{array}$ & $\begin{array}{l}\text { Singapour- } \\
\text { Corée }\end{array}$ & $\begin{array}{l}\text { Singapour- } \\
\text { Panama }\end{array}$ & $\begin{array}{c}\text { Accord } \\
\text { transpacifique }\end{array}$ & $\begin{array}{l}\text { Singapour- } \\
\text { Inde }\end{array}$ & TOTAL \\
\hline \multirow[t]{3}{*}{$\begin{array}{l}4 . \\
\text { Reconnaissance } \\
\text { (mutuelle) des } \\
\text { évaluations de la } \\
\text { conformité (suite) }\end{array}$} & $\begin{array}{l}\text { Impose-t-il aux } \\
\text { parties } \\
\text { d'expliquer leurs } \\
\text { raisons en cas de } \\
\text { non- } \\
\text { reconnaissance? }\end{array}$ & non & non & non & non & non & non & non & $\begin{array}{c}\text { oui, } \\
\text { également en } \\
\text { cas de refus } \\
\text { d'accréditation } \\
\text { d'un } \\
\text { organisme } \\
\text { d'évaluation } \\
\text { de la } \\
\text { conformité } \\
\end{array}$ & $\begin{array}{l}\text { oui, également } \\
\text { en cas de } \\
\text { refus } \\
\text { d'accréditation } \\
\text { d'un } \\
\text { organisme } \\
\text { d'évaluation de } \\
\text { la conformité }\end{array}$ & non & $\begin{array}{c}2 \text { sur } \\
10\end{array}$ \\
\hline & $\begin{array}{l}\text { S'accompagne-t- } \\
\text { il d'un accord de } \\
\text { reconnaissance } \\
\text { mutuelle } \\
\text { (ARM) distinct? }\end{array}$ & $\begin{array}{l}\text { oui, pour les } \\
\text { cosmétiques } \\
\text { et les } \\
\text { équipements } \\
\text { électriques et } \\
\text { électroniques }\end{array}$ & $\begin{array}{l}\text { oui, pour les } \\
\text { équipements } \\
\text { électriques et } \\
\text { électroniques }\end{array}$ & $\begin{array}{l}\text { oui, pour les } \\
\text { équipements } \\
\text { électriques }\end{array}$ & non & $\begin{array}{c}\text { oui, pour les } \\
\text { produits } \\
\text { alimentaires et } \\
\text { horticoles, les } \\
\text { équipements } \\
\text { électriques, } \\
\text { électroniques et de } \\
\text { télécommunications } \\
\text { et les produits } \\
\text { pharmaceutiques }\end{array}$ & $\begin{array}{l}\text { oui, pour les } \\
\text { équipements de } \\
\text { télécommunications } \\
\text { (ARM TEL de } \\
\text { I'APEC) }\end{array}$ & non & non & non & à venir & $\begin{array}{c}6 \text { sur } \\
10\end{array}$ \\
\hline & $\begin{array}{l}\text { Encourage-t-il la } \\
\text { conclusion d'un } \\
\text { ARM distinct? }\end{array}$ & oui & oui & oui & non & oui & oui & oui & non & non & oui & $\begin{array}{l}7 \text { sur } \\
10\end{array}$ \\
\hline \multirow[t]{2}{*}{ 5. Transparence } & $\begin{array}{l}\text { L'accord } \\
\text { contient-il des } \\
\text { dispositions sur } \\
\text { la transparence? }\end{array}$ & oui & oui & oui & oui & oui & oui & oui & oui & oui & oui & $\begin{array}{c}10 \text { sur } \\
10\end{array}$ \\
\hline & $\begin{array}{l}\text { Impose-t-il aux } \\
\text { membres } \\
\text { d'organiser des } \\
\text { consultations et } \\
\text { de notifier les } \\
\text { règlements et } \\
\text { procédures au } \\
\text { niveau bilatéral } \\
\text { ou régional avant } \\
\text { leur adoption? }\end{array}$ & oui & oui & oui & oui & oui & oui & oui & oui & oui & & $\begin{array}{c}10 \text { sur } \\
10\end{array}$ \\
\hline
\end{tabular}


TAD/TC/WP(2007)12/FINAL

\begin{tabular}{|c|c|c|c|c|c|c|c|c|c|c|c|c|}
\hline Catégories & Questions & ASEAN & $\begin{array}{l}\text { Singapour- } \\
\text { Nouvelle- } \\
\text { Zélande }\end{array}$ & $\begin{array}{l}\text { Singapour- } \\
\text { Japon }\end{array}$ & $\begin{array}{l}\text { Singapour- } \\
\text { AELE }\end{array}$ & $\begin{array}{l}\text { Singapour- } \\
\text { Australie }\end{array}$ & $\begin{array}{l}\text { Singapour- } \\
\text { États-Unis }\end{array}$ & $\begin{array}{l}\text { Singapour- } \\
\text { Corée }\end{array}$ & $\begin{array}{l}\text { Singapour- } \\
\text { Panama }\end{array}$ & $\begin{array}{c}\text { Accord } \\
\text { transpacifique }\end{array}$ & $\begin{array}{l}\text { Singapour- } \\
\text { Inde }\end{array}$ & TOTAL \\
\hline \multirow[t]{3}{*}{$\begin{array}{l}\text { 5. Transparence } \\
\text { (suite) }\end{array}$} & $\begin{array}{l}\text { Fixe-t-il un délai } \\
\text { de réception des } \\
\text { observations des } \\
\text { autres parties? }\end{array}$ & non & oui & oui & non & non & non & non & non & oui & oui & $\begin{array}{c}5 \text { sur } \\
10\end{array}$ \\
\hline & $\begin{array}{l}\text { Ce délai est-il } \\
\text { expressément } \\
\text { supérieur à } \\
60 \text { jours? }\end{array}$ & non & $\begin{array}{l}\text { non, « } 60 \\
\text { jours au } \\
\text { moins » }\end{array}$ & $\begin{array}{c}\text { non, le } \\
\text { délai n'est } \\
\text { pas } \\
\text { supérieur à } \\
60 \text { jours }\end{array}$ & non & non & non & non & non & $\begin{array}{c}\text { non, « } 60 \text { jours } \\
\text { au moins » }\end{array}$ & $\begin{array}{l}\text { non, « } 60 \\
\text { jours au } \\
\text { moins » }\end{array}$ & $\begin{array}{c}0 \text { sur } \\
10\end{array}$ \\
\hline & $\begin{array}{l}\text { Impose-t-il la } \\
\text { mise en place } \\
\text { d'un point } \\
\text { (distinct) } \\
\text { d'information? }\end{array}$ & oui & non & non & non & oui & oui & oui & non & non & oui & $\begin{array}{l}5 \text { sur } \\
10\end{array}$ \\
\hline $\begin{array}{l}\text { 6. Mise en œuvre et } \\
\text { règlement des } \\
\text { différends }\end{array}$ & $\begin{array}{l}\text { L'accord } \\
\text { préconise-t-il la } \\
\text { mise en place } \\
\text { d'un organisme } \\
\text { régional (par } \\
\text { exemple un } \\
\text { comité) sur les } \\
\text { OTC pour suivre } \\
\text { et examiner les } \\
\text { engagements } \\
\text { pris en matière } \\
\text { d'OTC et le } \\
\text { processus qui s'y } \\
\text { rapporte et les } \\
\text { invite-t-ili à y } \\
\text { participer? }\end{array}$ & oui & oui & oui & oui & oui & $\begin{array}{c}\text { oui } \\
\text { (coordinateurs } \\
\text { ) }\end{array}$ & oui & $\begin{array}{c}\text { oui } \\
\text { (coordinateurs) }\end{array}$ & oui & oui & $\begin{array}{c}10 \text { sur } \\
10\end{array}$ \\
\hline
\end{tabular}


TAD/TC/WP(2007)12/FINAL

\begin{tabular}{|c|c|c|c|c|c|c|c|c|c|c|c|c|}
\hline \multirow[t]{3}{*}{$\begin{array}{l}\text { 6. Mise en œuvre et } \\
\text { règlement des } \\
\text { différends (suite) }\end{array}$} & $\begin{array}{l}\text { Contient-il des } \\
\text { dispositions } \\
\text { relatives au } \\
\text { règlement des } \\
\text { différends à } \\
\text { l'échelon régional } \\
\text { en lien avec les } \\
\text { OTC? }\end{array}$ & oui & oui & oui & oui & oui & oui & oui & oui & oui & oui & $\begin{array}{c}10 \text { sur } \\
10\end{array}$ \\
\hline & $\begin{array}{l}\text { Prévoit-il } \\
\text { l'organisation de } \\
\text { consultations et } \\
\text { la formulation de } \\
\text { recommandations } \\
\text { ? }\end{array}$ & oui & oui & oui & oui & oui & oui & oui & oui & oui & oui & $\begin{array}{c}10 \text { sur } \\
10\end{array}$ \\
\hline & $\begin{array}{l}\text { Prévoit-il un } \\
\text { mécanisme plus } \\
\text { formel de } \\
\text { règlement des } \\
\text { différents à } \\
\text { l'échelon régional } \\
?\end{array}$ & oui & non & non & non & oui & non & non & $\begin{array}{c}\text { exclu de } \\
\text { l'accord } \\
\text { formel de } \\
\text { règlement des } \\
\text { différends }\end{array}$ & non & non & $\begin{array}{c}2 \text { sur } \\
10\end{array}$ \\
\hline \multirow[t]{2}{*}{$\begin{array}{l}\text { 7. Renforcement de } \\
\text { la coopération }\end{array}$} & $\begin{array}{l}\text { Contient-il des } \\
\text { dispositions } \\
\text { spécifiques } \\
\text { relatives à } \\
\text { l'assistance } \\
\text { technique? }\end{array}$ & oui & non & non & non & oui & non & non & non & non & oui & $\begin{array}{c}3 \text { sur } \\
10\end{array}$ \\
\hline & $\begin{array}{l}\text { Prévoit-il une } \\
\text { coopération en } \\
\text { matière de } \\
\text { métrologie? }\end{array}$ & $\begin{array}{c}\text { oui, } \\
\text { fondée } \\
\text { sur des } \\
\text { mesure } \\
\text { s } \\
\text { internat } \\
\text { ionales }\end{array}$ & non & non & non & non & non & non & non & non & non & $\begin{array}{c}1 \text { sur } \\
10\end{array}$ \\
\hline
\end{tabular}


TAD/TC/WP(2007)12/FINAL

Tableau 9. Tour d'horizon des dispositions juridiques contenues dans les ACR conclus par le Maroc

\begin{tabular}{|c|c|c|c|c|c|c|c|}
\hline Catégories & Questions & Maroc-UE & Maroc-AELE & Maroc-États-Unis & Maroc-Turquie & Accord d'Agadir & TOTAL \\
\hline \multirow[t]{2}{*}{$\begin{array}{l}\text { 1. Référence à } \\
\text { l'Accord sur les } \\
\text { OTC de l'OMC }\end{array}$} & $\begin{array}{l}\text { L'accord fait-il référence à } \\
\text { l'Accord de l'OMC (ses objectifs, } \\
\text { règles et/ou dispositions } \\
\text { provisions)? }\end{array}$ & non & oui & oui & oui & non & 3 sur 5 \\
\hline & $\begin{array}{l}\text { Le chapitre sur les OTC de } \\
\text { l'accord vise-t-il des objectifs } \\
\text { d'une plus grande portée? }\end{array}$ & $\begin{array}{l}\text { oui, meilleure } \\
\text { facilitation des } \\
\text { échanges }\end{array}$ & non & $\begin{array}{c}\text { oui, meilleure } \\
\text { facilitation des } \\
\text { échanges }\end{array}$ & non & non & $\underline{2}$ sur 5 \\
\hline \multirow[t]{3}{*}{ 2. Harmonisation } & $\begin{array}{l}\text { L'accord impose-t-il aux parties } \\
\text { ou les encourage-t-il à } \\
\text { harmoniser leurs règlements } \\
\text { techniques, normes et } \\
\text { procédures d'évaluation de la } \\
\text { conformité? }\end{array}$ & oui & $\begin{array}{l}\text { non (seule une } \\
\text { meilleure } \\
\text { coopération est } \\
\text { encouragée) }\end{array}$ & $\begin{array}{l}\text { non (seule une } \\
\text { meilleure } \\
\text { coopération est } \\
\text { encouragée) }\end{array}$ & $\begin{array}{l}\text { non (seule une } \\
\text { meilleure } \\
\text { coopération est } \\
\text { encouragée) }\end{array}$ & $\begin{array}{l}\text { non (seule une } \\
\text { meilleure coopération } \\
\text { est encouragée) }\end{array}$ & 1 sur 5 \\
\hline & $\begin{array}{l}\text { Encourage-t-il l'utilisation des } \\
\text { normes et guides } \\
\text { internationaux? }\end{array}$ & non & non & oui & non & oui & 2 sur 5 \\
\hline & $\begin{array}{l}\text { Encourage-t-il l'utilisation des } \\
\text { normes et guides régionaux? }\end{array}$ & oui (UE) & oui (UE) & non & oui (UE) & non & 3 sur 5 \\
\hline \multirow[t]{2}{*}{$\begin{array}{l}\text { 3. Acceptation des } \\
\text { règlements } \\
\text { techniques comme } \\
\text { équivalents }\end{array}$} & $\begin{array}{l}\text { L'accord impose-t-il aux parties } \\
\text { ou les encourage-t-il à accepter } \\
\text { comme équivalents les } \\
\text { règlements techniques et les } \\
\text { normes des autres parties? }\end{array}$ & non & non & $\begin{array}{l}\text { oui (les encourage } \\
\text { seulement) }\end{array}$ & non & non & 1 sur 5 \\
\hline & $\begin{array}{l}\text { Leur impose-t-il d'expliquer les } \\
\text { raisons pour lesquelles elles } \\
\text { n'acceptent pas l'équivalence des } \\
\text { règlements techniques des autres } \\
\text { parties? }\end{array}$ & non & non & non & non & non & 0 sur 5 \\
\hline \multirow[t]{2}{*}{$\begin{array}{l}\text { 4. Reconnaissance } \\
\text { (mutuelle) des } \\
\text { évaluations de la } \\
\text { conformité }\end{array}$} & $\begin{array}{l}\text { L'accord préconise-t-il une } \\
\text { reconnaissance (mutuelle) des } \\
\text { règlements techniques et des } \\
\text { normes? }\end{array}$ & non & non & non & non & non & 0 sur 5 \\
\hline & $\begin{array}{l}\text { L'accord préconise-t-il une } \\
\text { reconnaissance (mutuelle) des } \\
\text { résultats des évaluations de la } \\
\text { conformité ? }\end{array}$ & oui & non & $\begin{array}{l}\text { oui, par des } \\
\text { mécanismes très } \\
\text { variés }\end{array}$ & non & non & 2 sur 5 \\
\hline
\end{tabular}




\section{TAD/TC/WP(2007)12/FINAL}

\begin{tabular}{|c|c|c|c|c|c|c|c|}
\hline Catégories & Questions & Maroc-UE & Maroc-AELE & Maroc-États-Unis & Maroc-Turquie & Accord d'Agadir & TOTAL \\
\hline \multirow[t]{3}{*}{$\begin{array}{l}\text { 4. Reconnaissance } \\
\text { (mutuelle) des } \\
\text { évaluations de la } \\
\text { conformité }\end{array}$} & $\begin{array}{l}\text { Impose-t-il aux parties d'expliquer } \\
\text { leurs raisons en cas de non- } \\
\text { reconnaissance? }\end{array}$ & non & non & oui & non & non & 1 sur 5 \\
\hline & $\begin{array}{l}\text { S'accompagne-t-il d'un accord de } \\
\text { reconnaissance mutuelle } \\
\text { (ARM) distinct? }\end{array}$ & non & non & non & non & non & 0 sur 10 \\
\hline & $\begin{array}{l}\text { Encourage-t-il la conclusion d'un } \\
\text { ARM distinct? }\end{array}$ & oui & non & oui & non & non & $2 \operatorname{sur} 5$ \\
\hline \multirow[t]{5}{*}{ 5. Transparence } & $\begin{array}{l}\text { L'accord contient-il des } \\
\text { dispositions sur la transparence? }\end{array}$ & non & oui & oui & oui & non & 3 sur 5 \\
\hline & $\begin{array}{l}\text { Impose-t-il aux membres de } \\
\text { procéder à des consultations et } \\
\text { de notifier les règlements et } \\
\text { procédures au niveau bilatéral ou } \\
\text { régional avant leur adoption? }\end{array}$ & non & oui & oui & oui & non & 3 sur 5 \\
\hline & $\begin{array}{l}\text { Fixe-t-il un délai de réception des } \\
\text { observations des autres parties? }\end{array}$ & non & oui & oui & oui & non & 3 sur 5 \\
\hline & $\begin{array}{l}\text { Ce délai est-il expressément } \\
\text { supérieur à } 60 \text { jours? }\end{array}$ & non & $\begin{array}{c}\text { non, « } 60 \text { jours au } \\
\text { moins }\end{array}$ & $\begin{array}{c}\text { non, « } 60 \text { jours au } \\
\text { moins » }\end{array}$ & $\begin{array}{c}\text { non, « } 60 \text { jours au } \\
\text { moins » }\end{array}$ & non & 0 sur 5 \\
\hline & $\begin{array}{l}\text { Impose-t-il la mise en place d'un } \\
\text { point (distinct) d'information? }\end{array}$ & non & non & non & non & non & 0 sur 5 \\
\hline \multirow[t]{2}{*}{$\begin{array}{l}\text { 6. Mise en œuvre et } \\
\text { règlement des } \\
\text { différends }\end{array}$} & $\begin{array}{l}\text { L'accord préconise-t-il la mise en } \\
\text { place d'un organisme régional } \\
\text { (par exemple un comité) sur les } \\
\text { OTC et les invite-t-il à y } \\
\text { participer? }\end{array}$ & non & non & oui (coordinateurs) & non & non & 1 sur 5 \\
\hline & $\begin{array}{l}\text { Contient-il des dispositions } \\
\text { relatives au règlement des } \\
\text { différends à l'échelon régional en } \\
\text { lien avec les OTC? }\end{array}$ & non & oui & oui & oui & oui & 4 sur 5 \\
\hline
\end{tabular}


TAD/TC/WP(2007)12/FINAL

\begin{tabular}{|c|c|c|c|c|c|c|c|}
\hline Catégories & Questions & Maroc-UE & Maroc-AELE & Maroc-États-Unis & Maroc-Turquie & Accord d'Agadir & TOTAL \\
\hline \multirow[t]{2}{*}{$\begin{array}{l}\text { 6. Mise en œuvre et } \\
\text { règlement des } \\
\text { différends (suite) }\end{array}$} & $\begin{array}{l}\text { Prévoit-il la tenue de } \\
\text { consultations et la formulation de } \\
\text { recommandations? }\end{array}$ & non & oui & oui & oui & oui & 4 sur 5 \\
\hline & $\begin{array}{l}\text { Prévoit-il un mécanisme plus } \\
\text { formel de règlement des } \\
\text { différents à l'échelon régional ? }\end{array}$ & non & non & oui & non & non & 1 sur 5 \\
\hline \multirow[t]{2}{*}{$\begin{array}{l}\text { 7. Renforcement de } \\
\text { la coopération }\end{array}$} & $\begin{array}{l}\text { Contient-il des dispositions } \\
\text { spécifiques relatives à } \\
\text { l'assistance technique? }\end{array}$ & oui & non & oui & non & non & 2 sur 5 \\
\hline & $\begin{array}{l}\text { Prévoit-il une coopération en } \\
\text { matière de métrologie? }\end{array}$ & oui & non & non & non & non & 1 sur 5 \\
\hline
\end{tabular}


Tableau 10. Tour d'horizon des résultats agrégés des différents examens

\begin{tabular}{|c|c|c|c|c|c|}
\hline Catégories & Questions & Chili & Singapour & Maroc & $\begin{array}{l}\text { Moyenne des } \\
\text { études de cas }\end{array}$ \\
\hline \multirow[t]{2}{*}{$\begin{array}{l}\text { 1. Référence à l'Accord } \\
\text { sur les OTC de l'OMC }\end{array}$} & $\begin{array}{l}\text { L'accord fait-il référence à l'Accord } \\
\text { de l'OMC (ses objectifs, règles et/ou } \\
\text { dispositions)? }\end{array}$ & $100 \%$ & $100 \%$ & $60 \%$ & $86 \%$ \\
\hline & $\begin{array}{l}\text { Le chapitre sur les OTC de l'accord } \\
\text { vise-t-il des objectifs plus d'une plus } \\
\text { grande portée? }\end{array}$ & $30 \%$ & $50 \%$ & $40 \%$ & $40 \%$ \\
\hline \multirow[t]{3}{*}{ 2. Harmonisation } & $\begin{array}{l}\text { L'accord impose-t-il aux parties ou } \\
\text { les encourage-t-il à harmoniser leurs } \\
\text { règlements techniques, normes et } \\
\text { procédures d'évaluation de la } \\
\text { conformité ? }\end{array}$ & $50 \%$ & $70 \%$ & $20 \%$ & $47 \%$ \\
\hline & $\begin{array}{l}\text { Encourage-t-il l'utilisation des } \\
\text { normes et guides internationaux? }\end{array}$ & $80 \%$ & $90 \%$ & $40 \%$ & $70 \%$ \\
\hline & $\begin{array}{l}\text { Encourage-t-il l'utilisation des } \\
\text { normes et guides régionaux? }\end{array}$ & $0 \%$ & $30 \%$ & $60 \%$ & $30 \%$ \\
\hline \multirow[t]{2}{*}{$\begin{array}{l}\text { 3. Acceptation des } \\
\text { règlements techniques } \\
\text { comme équivalents }\end{array}$} & $\begin{array}{l}\text { L'accord impose-t-il aux parties ou } \\
\text { les encourage-t-il à accepter comme } \\
\text { équivalents les règlements } \\
\text { techniques et les normes des autres } \\
\text { parties? }\end{array}$ & $40 \%$ & $40 \%$ & $20 \%$ & $33 \%$ \\
\hline & $\begin{array}{l}\text { Leur impose-t-il d'expliquer les } \\
\text { raisons pour lesquelles elles } \\
\text { n'acceptent pas l'équivalence des } \\
\text { règlements techniques des autres } \\
\text { parties? }\end{array}$ & $70 \%$ & $30 \%$ & $0 \%$ & $33 \%$ \\
\hline \multirow[t]{5}{*}{$\begin{array}{l}\text { 4. Reconnaissance } \\
\text { (mutuelle) des } \\
\text { évaluations de la } \\
\text { conformité }\end{array}$} & $\begin{array}{l}\text { L'accord préconise-t-il une } \\
\text { reconnaissance (mutuelle) des } \\
\text { règlements techniques et des } \\
\text { normes? }\end{array}$ & $0 \%$ & $0 \%$ & $0 \%$ & $0 \%$ \\
\hline & $\begin{array}{l}\text { L'accord préconise-t-il une } \\
\text { reconnaissance (mutuelle) des } \\
\text { résultats des évaluations de la } \\
\text { conformité? }\end{array}$ & $90 \%$ & $100 \%$ & $40 \%$ & $77 \%$ \\
\hline & $\begin{array}{l}\text { Impose-t-il aux parties d'expliquer } \\
\text { leurs raisons en cas de non- } \\
\text { reconnaissance? }\end{array}$ & $60 \%$ & $20 \%$ & $20 \%$ & $33 \%$ \\
\hline & $\begin{array}{l}\text { S'accompagne-t-il d'un accord de } \\
\text { reconnaissance mutuelle } \\
\text { (ARM) distinct? }\end{array}$ & $10 \%$ & $60 \%$ & $0 \%$ & $23 \%$ \\
\hline & $\begin{array}{l}\text { Encourage-t-il la conclusion d'un } \\
\text { ARM distinct? }\end{array}$ & $70 \%$ & $70 \%$ & $40 \%$ & $60 \%$ \\
\hline \multirow[t]{3}{*}{ 5. Transparence } & $\begin{array}{l}\text { L'accord contient-il des dispositions } \\
\text { sur la transparence? }\end{array}$ & $80 \%$ & $100 \%$ & $60 \%$ & $80 \%$ \\
\hline & $\begin{array}{l}\text { Impose-t-il aux membres de } \\
\text { procéder à des consultations et de } \\
\text { notifier les règlements et procédures } \\
\text { au niveau bilatéral ou régional avant } \\
\text { leur adoption? }\end{array}$ & $80 \%$ & $100 \%$ & $60 \%$ & $80 \%$ \\
\hline & $\begin{array}{l}\text { Fixe-t-il un délai de réception des } \\
\text { observations des autres parties ? }\end{array}$ & $70 \%$ & $50 \%$ & $60 \%$ & $60 \%$ \\
\hline
\end{tabular}


TAD/TC/WP(2007)12/FINAL

\begin{tabular}{|l|l|c|c|c|c|}
\hline \multicolumn{1}{|c|}{ Catégories } & \multicolumn{1}{|c|}{ Questions } & Chili & Singapour & Maroc & $\begin{array}{c}\text { Moyenne des } \\
\text { études de cas }\end{array}$ \\
\hline $\begin{array}{l}\text { 5. Transparence } \\
\text { (suite) }\end{array}$ & $\begin{array}{l}\text { Ce délai est-il expressément } \\
\text { supérieur à 60 jours ?' }\end{array}$ & $0 \%$ & $0 \%$ & $0 \%$ & $0 \%$ \\
\hline $\begin{array}{l}\text { Impose-t-il la mise en place d'un } \\
\text { point (distinct) d'information? }\end{array}$ & $0 \%$ & $50 \%$ & $0 \%$ & $17 \%$ \\
\hline $\begin{array}{l}\text { 6. Mise en œuvre et } \\
\text { règlement des } \\
\text { différends }\end{array}$ & $\begin{array}{l}\text { L'accord préconise-t-il la mise en } \\
\text { place d'un organisme régionale (par } \\
\text { exemple un comité) sur les OTC } \\
\text { pour suivre et examiner les } \\
\text { engagements pris en matière d'OTC } \\
\text { et le processus qui s'y rapporte et } \\
\text { les invite-t-il à y participer ? }\end{array}$ & $100 \%$ & $100 \%$ & $20 \%$ & $73 \%$ \\
\hline & $\begin{array}{l}\text { Contient-il des dispositions relatives } \\
\text { au règlement des différends à } \\
\text { l'échelon régional en lien avec les } \\
\text { OTC ? }\end{array}$ & $80 \%$ & $100 \%$ & $80 \%$ & $87 \%$ \\
\hline $\begin{array}{l}\text { Prévoit-il la tenue de consultations et } \\
\text { la formulation de recommandations? }\end{array}$ & $70 \%$ & $100 \%$ & $80 \%$ & $83 \%$ \\
\hline 7. Renforcement de la la \\
coopération & $\begin{array}{l}\text { Prévoit-il un mécanisme plus formel } \\
\text { de règlement des différents à } \\
\text { l'échelon régional ? }\end{array}$ & $20 \%$ & $20 \%$ & $20 \%$ & $20 \%$ \\
\hline $\begin{array}{l}\text { Contient-il des dispositions } \\
\text { spécifiques relatives à l'assistance } \\
\text { technique? }\end{array}$ & $\begin{array}{l}\text { Prévoit-il une coopération en matière } \\
\text { de métrologie? }\end{array}$ & $40 \%$ & $10 \%$ & $20 \%$ & $23 \%$ \\
\hline
\end{tabular}

Note 1 : Ici, « 60 jours au moins » ne peut signifier supérieur à 60 jours.

Note 2 : On entend ici par « coordinateurs » un organisme mise en place pour suivre la mise en œuvre des dispositions relatives aux OTC. 